\title{
ANALYTICAL SUMMARY OF EXPERIMENTAL DATA FROM TWO YEARS OF HOURLY SEQUENTIAL PRECIPITATION SAMPLES AT BROOKHAVEN NATIONAL LABORATORY
}

Gilbert S. RAYNOR and Janet V. HAYeS

May 1979

\section{ATMOSPHERIC SCIENCES DIVISION DEPARTMENT OF ENERGY AND ENVIRONMENT}

BROOKHAVEN NATIONAL LABORATORY ASSOCIATED UNIVERSITIES, INC. 


\section{DISCLAIMER}

This report was prepared as an account of work sponsored by an agency of the United States Government. Neither the United States Government nor any agency Thereof, nor any of their employees, makes any warranty, express or implied, or assumes any legal liability or responsibility for the accuracy, completeness, or usefulness of any information, apparatus, product, or process disclosed, or represents that its use would not infringe privately owned rights. Reference herein to any specific commercial product, process, or service by trade name, trademark, manufacturer, or otherwise does not necessarily constitute or imply its endorsement, recommendation, or favoring by the United States Government or any agency thereof. The views and opinions of authors expressed herein do not necessarily state or reflect those of the United States Government or any agency thereof. 


\section{DISCLAIMER}

Portions of this document may be illegible in electronic image products. Images are produced from the best available original document. 
BNL 51058

UC-11

MASTER

(Environmental Control Technology and

Earth Sciences - TID-4500)

\section{ANALYTICAL SUMMARY OF EXPERIMENTAL DATA FROM TWO YEARS OF HOURLY SEQUENTIAL PRECIPITATION SAMPLES AT BROOKHAVEN NATIONAL LABORATORY}

Gilbert S. Raynor and Janet V. Hayes

May 1979

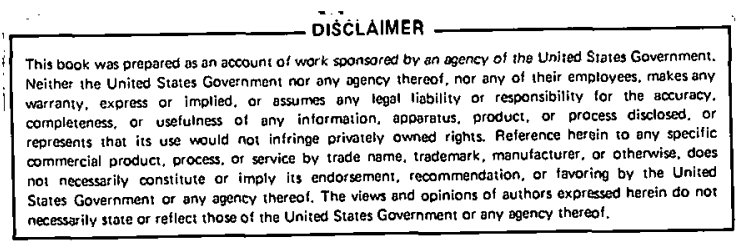

ATMOSPHERIC SCIENCES DIVISION DEPARTMENT OF ENERGY AND ENVIRONMENT

BROOKHAVEN NATIONAL LABORATORY UPTON, NEW YORK 11973 


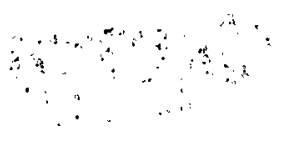

NOT I C E

This rcport was prepared as an account of work sponsored by the United States Government. Neither the United States nor the United States Department of Energy (DOE), nor any of their employees, nor any of their contractors, subcontractors, or their employees, makes any warranty, express or implied, or assumes any legal liability us icspinsinsility for the accuracy, completencos or uocfulneso of any infortination, apparatus, product or process disclosed, or represents that its use would not infringe privately owned rights.

Printed in the United States of America

Available from

National Technical Information Service

U.S. Department of Commerce

5285 Port Royal Road

Springfield, VA 22161

Price: Printed Copy $\$ 6.00$; Microfiche $\$ 3.00$

September 1979

365 copies 
Hourly precipitation samples were collected at Brookhaven National Laboratory from June 1976 through May 1978 with the Brookhaven Automatic Sequential Precipitation Sampler. Conventional meteorological data were recorded for each sample period. The samples were analyzed for $\mathrm{pH}$, conductivity, and concentrations of sulfate, nitrogen, ammonium, sodium, and chloride ions. Ratios of selected ions, excess conductivity above the $\mathrm{H}^{+}$contribution, and excess sulfate and chloride above the seawater ratio were calculated and treated as additional variables. Statistical analyses were performed of the relationships between selected chemical variables and between them and meteorological conditions. Rainfall rate was also analyzed in terms of other meteorological variables. Because a number of units were changed after publication of an earlier report (BNL 50826), the results (presented in a series of tables) are given for each year separately and for both years jointly. 


\section{İNTRODUCTION}

An earlier report ${ }^{1}$ presented a statistical analysis of the first year (June 1,1976 , to May 31,1977 ) of experimental data obtained from the sequential precipitation sampling program at Brookhaven National Laboratory (BNL). Because of significant seasonal variation in precipitation chemistry, data are analyzed only in increments of complete years. "This report contains an analysis of the first two years of data (June 1, 1976, to May 31, 1978). Results from the two years are presented both separately and jointly for two reasons: (1) Units have been changed since the earlier report, ${ }^{1}$ which makes direct comparisons difficult. (2). Comparison of the separate data from the two years gives some indication of the variability from year to year both in the frequency of meteorological conditions and in concentrations of precipitation contaminants.

This report again presents the relationships among selected chemical variables and also those between the chemical and meteorological variables. During the second year, improved analytical techniques made it possible to obtain at least some measurements from smaller samples. Thus, the number of cases in the second year was larger but the number of actual precipitation hours, although also larger, did not increase in the same proportion. Only a few precipitation hours were lost in both years because of mechanical, electrical, or laboratory problems; therefore, the data base includes nearly all possible hours during the period having precipitation amounts great enough for chemical analysis of the samples. This report has been prepared for the benefit of other users and as a data base for further analytical studies, which will be presented elsewhere. These include an analysis of hourly variability in precipitation chemistry compared with event averages, and a study of temporal changes in precipitation chemistry within events and their relationsh1p to meteorological changes. 
BNL is a station in the MAP3S precipitation chemistry network, and samples have been taken on an event basis also with a Battelle. Precipitation Collector 2 since February 1978. A comparison of results from the two samplers is in preparation and will be published separately.

\section{EXPERIMENTAL METHODS}

Precipitation samples were taken with the Brookhaven Automatic Sequential Precipitation Sampler previously described. 3,4 Sampling techniques were not changed.

Samples continued to be analyzed by the Analytical Chemistry Group, Division. of Environmental Chemistry, Department of Energy and Environment, by the procedures described earlier ${ }^{1}$ except for the following modification. Since February 6, 1978, all so $_{4}^{2-}$ analyses have been made with the methylthymol blue colorimetric procedure, which is more sensitive than the turbidimetric procedure.

The methylthymol blue procedure for $\mathrm{So}_{4}{ }^{2-}$ is automated and run on the Auto Analyzer. II. The rain sample is passed through a cation exchange column. to remove interfering cations. The sulfate in the solution then reacts with barium chloride at a $\mathrm{pH}$ of 2.5 to 3.0 to form barium sulfate. The $\mathrm{pH}$ is adjusted to 12.5 to 13.0 , and the excess barium reacts with methylthymol blue. Initially, the barium chloride and methylthymol blue are equimolar and equivalent to the highest concentration of sulfate ion expected. Thus the amount of uncomplexed methyl.thymol blue, whose absorbance is measured at $460 \mathrm{~nm}$, is equal to the amount of sulfate present in the sample.

\section{DATA}

Data recorded for each hourly sample included date, sample number, time of beginning and end of sample, duration of period with the cover open, precipitation 
event number within the month, precipitation amount, precipitation type, cloud type, synoptic type causing the precipitation, wind direction and speed at a height of $108 \mathrm{~m}$, air temperature, $\mathrm{pH}$ and conductivity of the sample, and concentrations of nitrate plus nitrite, sulfate, ammonium, chloride, and sodium ions. The sum of the nitrate and nitrite ions is referred to below as nitrogen. (N). In the earlier report, ${ }^{1}$ "nitrate" as used in table headings also included nitrite and should be considered nitrogen.

The meteorological parameters are self-explanatory except for the following cases. In precipitation type, thunderstorm hours include all hours in which a thunderstorm occurred even though the thunderstorm did not last the entire hour. They also include hours. with hail whether or not thunder or lightning was observed. Snow includes all completely frozen precipitation such as snow showers. Sleet includes mixed. snow and sleet and freezing rain. Mixed precipitation includes rain and snow, rain and:sleet, rain, and freezing rain and rainshowers plus snow showers in the same hour. Hours with drizzle and freezing drizzle seldom give a sample large enough to analyze and would be added to rain or freezing rain respectively if they occurred.

Under synoptic type, low includes all cases in which the precipitation could not be attributed to a specific type of front. Cold front precipitation includes precipitation both ahead of and behind the front. Squall lines include pre-coldfrontal troughs.

Measurements of temperature were converted from ${ }^{\circ} \mathrm{F}$ to ${ }^{\circ} \mathrm{C}$, and a new temperature classification, based on $5^{\circ} \mathrm{C}$ instead of $10^{\circ} \mathrm{F}$ intervals, was selected. Measurements of precipitation were converted from inches to $\mathrm{mm}$. The new precipitation amount classification selected is nearly equivalent to the one used earlier. 1 Unt ts of concentration of the ions $\mathrm{SO}_{4}{ }^{2-}, \mathrm{NO}_{2}^{-}+\mathrm{NO}_{3}^{-}, \mathrm{NH}_{4}^{+}, \mathrm{Cl}^{-}$, and $\mathrm{Na}^{+}$were converted from ppm to microequivalents/liter by using the following multiplication 
factors: $\operatorname{so}_{4}^{2-}, 20.82 ; \mathrm{N}, 71.38 ; \mathrm{NH}_{4}^{+}, 55.46 ; \mathrm{Cl}^{-}, 28.21 ; \mathrm{Na}^{+}, 43.48$. Units of $\mathrm{H}^{+}$previously calculated from $\mathrm{pH}$ were converted from molar concentration to

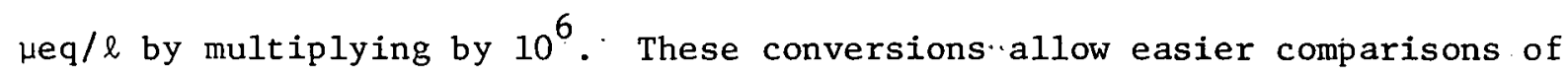
ionic concentrations and facilitate computations of ion balance.

Certain derived parameters were calculated from the original data. The followịng ratios of ionic species were computed: $\mathrm{so}_{4}^{2-} / \mathrm{Cl}^{-}, \mathrm{so}_{4}^{2-} / \mathrm{Na}^{+}, \mathrm{N}^{2} \mathrm{SO}_{4}{ }^{2-}$, $\mathrm{NH}_{4}^{+} / \mathrm{N}, \mathrm{NH}_{4}^{+} / \mathrm{SO}_{4}^{2-}, \mathrm{Cl}^{-} / \mathrm{Na}^{+}$.

Excess conductivity was calculated to give a measure of conductivity attributable to constituents other than $\mathrm{H}^{+}$. In computing conductivity of the measured constituents, the following values of conductivity '(umhos/cm) were assigned.for each $\mu$ eq/ $/$ of the ion: $\mathrm{H}^{+}, 0.329 ; \mathrm{SO}_{4}^{2-}, 0.073 ; \mathrm{N}, 0.064 ; \mathrm{NH}_{4}^{+}$, $0.068 ; \mathrm{Cl}^{-}, 0.068 ; \mathrm{Na}^{+}, 0.046$. In about $9 \%$ of al1 cases, for reasons not apparent, a deficit (usually small) was found instead of an excess. This is contrary to expectations since not all the possible ions in the samples are even measured. Reasons for these discrepancies are being sought.

Excess sulfate and excess chloride, that is, the non-seawater contributions of these of these lons, were calculated by using the $\mathrm{SO}_{4}{ }^{2-} / \mathrm{Na}^{+}$, and $\mathrm{Cl}^{-} / \mathrm{Na}^{+}$ratios in seawater to compute the seawater contribution. Sodium was used as a reference because the $\mathrm{Cl}^{-} / \mathrm{Na}^{+}$ratios in the precipitation samples are usually higher than the ratio in seawater, suggesting sources of $\mathrm{Cl}^{-}$other than the ocean. In addition, this area, in contrast to some inland locations, is thought to have no other significant sources of sodium. Seawater ratios were obtained from the composition of seawater as given in a standard reference ${ }^{5}$ in units of $\mathrm{g} / \mathrm{kg}$ as Eollows: $\mathrm{SO}_{4}^{2-} / \mathrm{Na}^{+}=0.252, \mathrm{Cl}^{-} / \mathrm{Na}^{+}=1: 800$. In units of $\mu \mathrm{eq} / \ell$, these ratios are 0.121 and 1.167 respectively. 
In two of 1346 cases, a deficit rather than an excess of $\mathrm{sO}_{4}^{2-}$ was found with reference to the seawater ratio. In $21 \%$ of all cases, a deficit of $\mathrm{Cl}^{-}$ rather than an excess was found but it was usually small.

In computing excesses, ratios, and correlation and regression relationships, values in $\mu$ eq/ $\ell$ less than the following were excluded because they are near the lower limit of the measurement capability: $\mathrm{SO}_{4}^{2-}, 20 ; \mathrm{N}, 4 ; \mathrm{NH}_{4}^{+} ; 3$; $\mathrm{Cl}^{-}, 17 ; \mathrm{Na}^{+} ; 4$. In ppm these values are about $1.0,0.1,0.06,0.6$, and 0.1 respectively.

\section{DATA ANALYSIS}

Analytical procedures and methods of data presentation were selected so that the results would have maximum usefulness to the greatest number of potential users. Statistical analyses were made by an electronic computer with extensive use of the Statistical Package for the Social Sciences, ${ }^{6}$ which eliminated much programming and facilitated computations.

Chemical parameters of the samples: and ratios between them were related by standard linear correlation and regression techniques: The relationship of chemical speciès to meteorological conditions was determined by categorizing.: the chemical data by meteorological parameters and computing the mean, standard. deviation, and other statistics of the resulting distributions.

For these same: categories, a weighted mean was computed by weighting the value of each chemical parameter by the amount of precipitation in that hour. In the lew custs ul samples representing greater or less than one hour of collection, the dala were normalized by time: also. The weighted mean values approximate : the values that would be obtained if all samples taken under a selected condition were mixed and a singlc analysis performed. These means are most. 
appropriate for relating precipitation chemistry to long-term effects such as effects on soils or groundwater. The unweighted means give greater importance to extreme events and are more appropriate for relating the chemistry of a category to short-term effects such as plant damage by "acid rain."

An analysis of hourly average precipitation rate in terms of other meteorological variables is also included.

Nearly all data were used except for a few cases removed because of probable errors in chemical measurements. Several hours of data during the peak of Hurricane Belle were removed from all analyses because they had concen* trations of chloride and sodfum about three orders of uagnitude above normal.

Many samples were too small for all the desired chemical analyses, and some analyses require greater volumes of water than others; therefore, the number of cases differs from one measurement to another. .

\section{. RESULTS}

Results are presented here in tabular form. Units are all in $\mu$ eq/ $\ell$ except $\mathrm{pH}$ and conductivity ( $\mu \mathrm{mhos} / \mathrm{cm})$. Ratios are nond1mensiunal. Graphical presentation of selected results will be included in future analytical papers. The tables are largely self-explanatory but are described briefly below. Relationships between chemical parameters

The relationships between selected chemical parameters are shown in Table 1, which gives the slope and intercept of the linear regression equations relating the variables and the linear correlation coefficient between the variables. Where one variable could be considered dependent and the other independent, the former was used as the y variable and the latter as the $x$ variable; where no such relationship was presumed, the choice of $x$ and $y$ variables was arbitrary. Some pairs of varlables show varying degrees of 
positive correlation. This does not necessarily imply a physical cause and effect since both may be related to some other variable. Other pairs show no correlation; the results are included to document this.

The variability of selected chemical parameters by $\mathrm{pH}$ class is shown in Table 2. Included for each class are weighted mean, mean, standard deviation, minimum, maximum, and number of cases. The negative values in Tables $2 b ; \because:$ $2 d$, and $2 i$ are due to cases in which deficits rather than the expected excesses occurred. In the earlier report, ${ }^{1}$ cases with negative values were interpreted as having no excess and asșigned a zẹro value.

Relationship of chemical to meteorological parameters

Tables. 3 through 19 present the means and statistics of the chemical variables for all cases combined and for cases classified by the following. meteorological variables: seașon; synoptic type; precipitation type, precipitation rate, wind speed, wind direction; and air temperature. The negative values in Tables 4,8 , and 13 have the șame explanation as those in Table 2 . Relationship of rainfalli rate to other meteorological parameters

Table 20 presents the means and statistics of precipitation rate classified by other meteorological variables.

\section{ACKNOWLEDGMENTS}

This study would have been impossible without the participation and cooperation of many people. B. Manowitz, L. Newman, and L. Evans participated in the original design of the project. P. Michael and G. Hendrey gave continued support. J. McNeil assisted in the desigin and construction of the sampler. He and R. Petty were primarily responsible for its operation and for collection of the samples. Chemical analyses were performed under the direction of L. Newman by M. Kinsley, C. Dudge, T. McGee, D. Spandau, and R. Wilson. 
1. Raynor, G. S. and Hayes, J. V., Experimental Data from Analysis of Sequential Precipitation Samples at Brookhaven National Laboratory. BNL $50.826, \cdot 1978$.

2. The MAP3S Precipitation Chemistry Network, First Periodic Summary Report (September 1976 - June 1977), PNL-2402, Pacific Northwest Laboratories, Richland, WA, 1977.

3. Raynor, G. S, and McNeil, J. P., The Brookhaven Automatic Sequential Precipitation Sampler, BNL 50818,1978 .

4. Raynor, G. S. and McNeil, J. P., An Lutomatic Sequential Precipitation Sampler, Atmos. Environ. 13, 149-55 (1979).

5. Wallace, W. J., The Development of the Chlorinity-Salinity Concept in Oceanography, Elsevier, New York; 1974.

6. Nie, N. H., Hul1, C. H., Jenkins, J. G., Steinbrenner, K, , and Bent, D. H., Statistical Package for the Social Sciences, 2nd Ed., McGraw-Hill, New York, $19 / 3$. 
TABLE 1 Correlation and Regression Analyses

of Relationships between Chemical Variables

(see text for units)

\begin{tabular}{|c|c|c|c|c|c|}
\hline Variable $y$ & Variable $x$ & Year & Intercept & Slope & $\begin{array}{l}\text { Correlatio } \\
\text { Coefficien }\end{array}$ \\
\hline Cond. & $\mathrm{H}^{+}$ & $\begin{array}{c}1 \\
2 \\
1 \& 2\end{array}$ & $\begin{array}{l}17.09 \\
16.20 \\
16.62\end{array}$ & $\begin{array}{l}0.28 \\
0.30 \\
0.29\end{array}$ & $\begin{array}{l}0.67 \\
0.60 \\
0.63\end{array}$ \\
\hline Cond. & $\mathrm{SO}_{4}^{2-}$ & $\begin{array}{c}1 \\
2 \\
1 \& 2\end{array}$ & $\begin{array}{r}-1.67 \\
5.82 \\
3.00\end{array}$ & $\begin{array}{l}0.39 \\
0.37 \\
0.37\end{array}$ & $\begin{array}{l}0.85 \\
0.84 \\
0.84\end{array}$ \\
\hline Cond. & $\left(\mathrm{NO}_{3}^{-}+\mathrm{NO}_{2}^{-}\right)$ & $\begin{array}{c}1 \\
2 \\
1 \& 2\end{array}$ & $\begin{array}{l}12.35 \\
16.18 \\
14.86\end{array}$ & $\begin{array}{l}0.50 \\
0.43 \\
0.45\end{array}$ & $\begin{array}{l}0.71 \\
0.65 \\
0.67\end{array}$ \\
\hline Cond. & $\mathrm{NH}_{4}^{+}$ & $\begin{array}{c}1 \\
2 \\
1 \& 2\end{array}$ & $\begin{array}{l}16.30 \\
18.95 \\
18.26\end{array}$ & $\begin{array}{l}0.65 \\
0.79 \\
0.71\end{array}$ & $\begin{array}{l}0.51 \\
0.49 \\
0.49\end{array}$ \\
\hline Cond. & $\mathrm{Na}^{+}$ & $\begin{array}{c}1 \\
2 \\
1 \& 2\end{array}$ & $\begin{array}{l}21.46 \\
20.31 \\
21.17\end{array}$ & $\begin{array}{l}0.18 \\
0.12 \\
0.13\end{array}$ & $\begin{array}{l}0.55 \\
0.66 \\
0.60\end{array}$ \\
\hline Cond. & $\mathrm{Cl}^{-}$ & $\begin{array}{c}1 \\
2 \\
1 \& 2\end{array}$ & $\begin{array}{l}21.19 \\
20.57 \\
20.79\end{array}$ & $\begin{array}{l}0.13 \\
0.12 \\
0.12\end{array}$ & $\begin{array}{l}0.55 \\
0.57 \\
0.56\end{array}$ \\
\hline Cond. & EX. $\mathrm{Cl}^{-}$ & $\begin{array}{c}1 \\
2 \\
1 \& 2\end{array}$ & $\begin{array}{l}32.01 \\
34.39 \\
35.72\end{array}$ & $\begin{array}{r}0.27 \\
-0.10 \\
0.01\end{array}$ & $\begin{array}{r}0.33 \\
-0.17 \\
0.02\end{array}$ \\
\hline Cond. & Precip. Rate & $\begin{array}{c}1 \\
2 \\
1 \& 2\end{array}$ & $\begin{array}{l}30.14 \\
35.11 \\
32.65\end{array}$ & $\begin{array}{l}-0.26 \\
-1.84 \\
-0.94\end{array}$ & $\begin{array}{l}-0.04 \\
-0.17 \\
-0.11\end{array}$ \\
\hline Ex. Cond. & $\mathrm{su}_{4}^{2-}$ & $\begin{array}{c}1 \\
2 \\
1 \& 2\end{array}$ & $\begin{array}{l}5.14 \\
6.62 \\
5.98\end{array}$ & $\begin{array}{l}0.12 \\
0.13 \\
0.12\end{array}$ & $\begin{array}{l}0.35 \\
0.37 \\
0.36\end{array}$ \\
\hline EX. Cond. & $\left(\mathrm{NO}_{3}^{-}+\mathrm{NO}_{2}^{-}\right)$ & $\begin{array}{c}1 \\
2 \\
1 \& 2\end{array}$ & $\begin{array}{l}9.90 \\
9.85 \\
9.83\end{array}$ & $\begin{array}{l}0.14 \\
0.16 \\
0.15\end{array}$ & $\begin{array}{l}0.28 \\
0.29 \\
0.29\end{array}$ \\
\hline EX. Cond. & $\mathrm{NH}_{4}^{+}$ & $\begin{array}{c}1 \\
2 \\
1 \& 2\end{array}$ & $\begin{array}{l}11.46 \\
10.00 \\
10.72\end{array}$ & $\begin{array}{l}0.19 \\
0.35 \\
0.27\end{array}$ & $\begin{array}{l}0.20 \\
0.27 \\
0.24\end{array}$ \\
\hline
\end{tabular}


TABLE 1 (continued) Chemical Variables

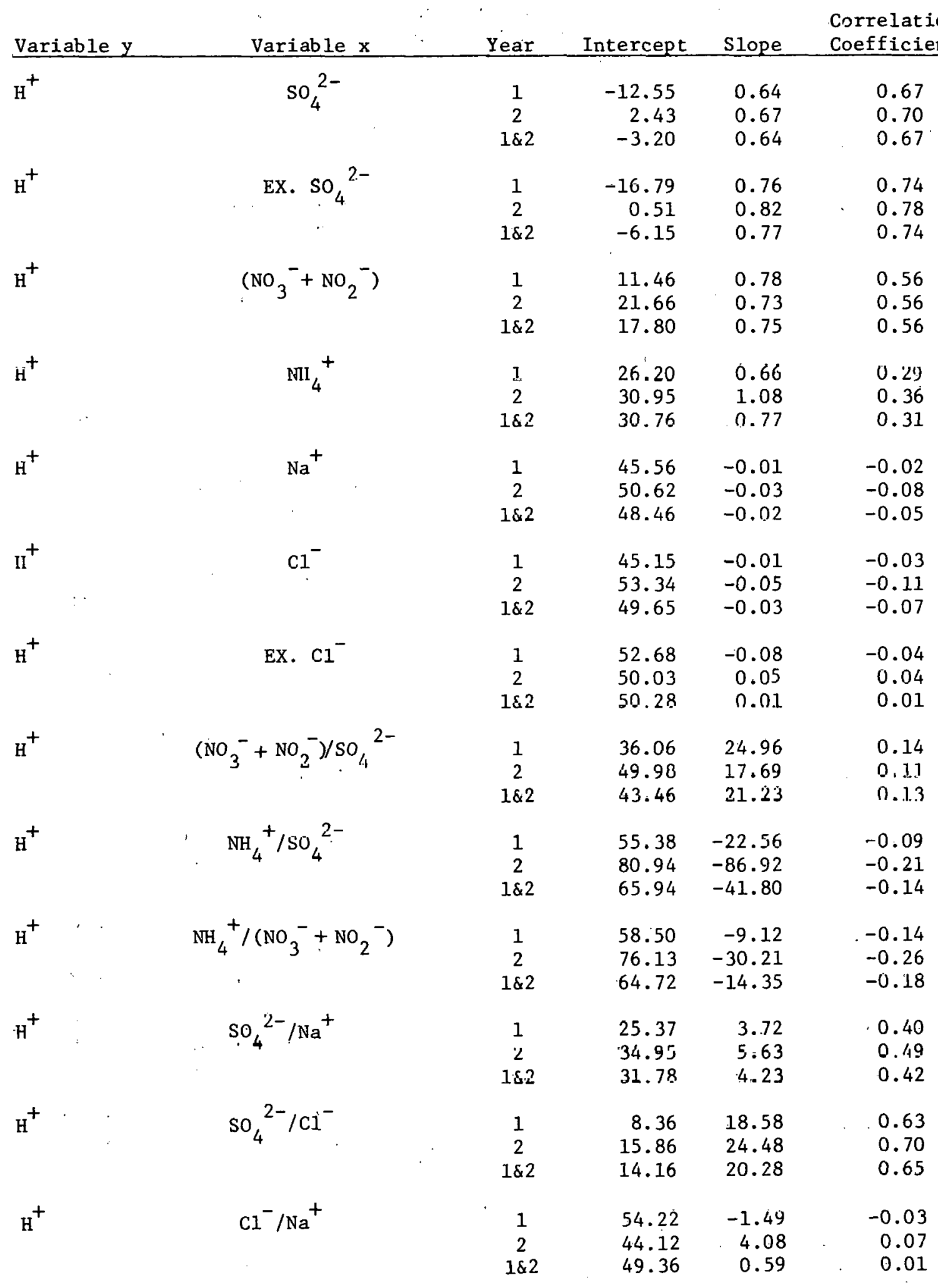


TABLE 1 (continued) Chemical Variables

\begin{tabular}{|c|c|c|c|c|c|}
\hline Variable y & Variable $x$ & Year & Intercept & S1ope & $\begin{array}{l}\text { Correlation } \\
\text { Coefficient } \\
\end{array}$ \\
\hline $\mathrm{H}^{+}$ & Prectp. Rate & $\begin{array}{c}1 \\
2 \\
1 \& 2\end{array}$ & $\begin{array}{l}40.56 \\
41.26 \\
41.31\end{array}$ & $\begin{array}{l}1.08 \\
2.08 \\
1.50\end{array}$ & $\begin{array}{l}0.06 \\
0.10 \\
0.08\end{array}$ \\
\hline $\mathrm{so}_{4}^{2-}$ & $\left(\mathrm{NO}_{3}^{-}+\mathrm{NO}_{2}^{-}\right)$ & $\begin{array}{c}1 \\
2 \\
1 \& 2\end{array}$ & $\begin{array}{l}45.73 \\
39.84 \\
42.22\end{array}$ & $\begin{array}{l}0.98 \\
0.99 \\
0.99\end{array}$ & $\begin{array}{l}0.67 \\
0.66 \\
0.66\end{array}$ \\
\hline $\mathrm{SO}_{4}^{2-}$ & $\mathrm{NH}_{4}^{+}$ & $\begin{array}{c}1 \\
2 \\
1 \& 2\end{array}$ & $\begin{array}{l}49.93 \\
36.95 \\
44.11\end{array}$ & $\begin{array}{l}1.45 \\
2.21 \\
1.75\end{array}$ & $\begin{array}{l}0.61 \\
0.68 \\
0.64\end{array}$ \\
\hline $\mathrm{SO}_{4}^{2-}$ & Precip. Rate & $\begin{array}{c}1 \\
2 \\
1 \& 2\end{array}$ & $\begin{array}{l}91.85 \\
87.89 \\
88.34\end{array}$ & $\begin{array}{l}-1.65 \\
-5.01 \\
-2.92\end{array}$ & $\begin{array}{l}-0.09 \\
-0.19 \\
-0.13\end{array}$ \\
\hline EX. SO $_{4}^{2-}$ & $\left(\mathrm{NO}_{3}^{-}+\mathrm{NO}_{2}^{-}\right)$ & $\begin{array}{c}1 \\
2 \\
1 \& 2\end{array}$ & $\begin{array}{l}43.01 \\
29.06 \\
35.45\end{array}$ & $\begin{array}{l}0.94 \\
1.11 \\
1.03\end{array}$ & $\begin{array}{l}0.64 \\
0.73 \\
0.69\end{array}$ \\
\hline EX. $\mathrm{SO}_{4}^{2-}$ & $\cdot \mathrm{NH}_{4}^{+}$ & $\begin{array}{c}1 \\
2 \\
1 \& 2\end{array}$ & $\begin{array}{l}39.72 \\
31.39 \\
36.38\end{array}$ & $\begin{array}{l}1.73 \\
2.24 \\
1.91\end{array}$ & $\begin{array}{l}0.61 \\
0.65 \\
0.63\end{array}$ \\
\hline$\left(\mathrm{NO}_{3}^{-}+\mathrm{NO}_{2}^{-}\right)$ & $\mathrm{NH}_{4}^{+}$ & $\begin{array}{c}1 \\
2 \\
1 \& 2\end{array}$ & $\begin{array}{l}16.00 \\
17.42 \\
16.87\end{array}$ & $\begin{array}{l}1.01 \\
0.96 \\
0.98\end{array}$ & $\begin{array}{l}0.61 \\
0.50 \\
0.55\end{array}$ \\
\hline$\left(\mathrm{NO}_{3}^{-}+\mathrm{NO}_{2}^{-}\right)$ & Precip. Rate & $\begin{array}{c}1 \\
2 \\
1 \& 2\end{array}$ & $\begin{array}{l}46.87 \\
42.82 \\
43.66\end{array}$ & $\begin{array}{l}-2.24 \\
-3.74 \\
-2.83\end{array}$ & $\begin{array}{l}-0.18 \\
-0.22 \\
-0.19\end{array}$ \\
\hline $\mathrm{Na}^{+}$ & $\mathrm{Cl}^{-}$ & $\begin{array}{c}1 \\
2 \\
1 \& 2\end{array}$ & $\begin{array}{r}-1.49 \\
0.27 \\
0.92\end{array}$ & $\begin{array}{l}0.71 \\
0.91 \\
0.81\end{array}$ & $\begin{array}{l}0.97 \\
0.94 \\
0.94\end{array}$ \\
\hline$\left(\mathrm{NO}_{3}{ }^{-}+\mathrm{NO}_{2}{ }^{-}\right) / \mathrm{SO}_{4}{ }^{2-}$ & Precip. Rate & $\begin{array}{c}1 \\
\dot{2} \\
1 \& 2\end{array}$ & $\begin{array}{l}0.52 \\
0.53 \\
0.53\end{array}$ & $\begin{array}{l}-0.02 \\
-0.02 \\
-0.02\end{array}$ & $\begin{array}{l}-0.14 \\
-0.13 \\
-0.13\end{array}$ \\
\hline $\mathrm{H}^{+}+\mathrm{NH}_{4}^{+}$ & $\mathrm{SO}_{4}^{2-}$ & $\begin{array}{c}1 \\
2 \\
1 \& 2\end{array}$ & $\begin{array}{r}-11.16 \\
1.70 \\
-3.54\end{array}$ & $\begin{array}{l}0.93 \\
0.90 \\
0.90\end{array}$ & $\begin{array}{l}0.79 \\
0.80 \\
0.79\end{array}$ \\
\hline $\mathrm{H}^{+}+\mathrm{NH}_{4}^{+}$ & $\left(\mathrm{NO}_{3}^{-}+\mathrm{NO}_{2}^{-}\right)$ & $\begin{array}{c}1 \\
2 \\
1 \& 2\end{array}$ & $\begin{array}{l}20.58 \\
28.24 \\
25.15\end{array}$ & $\begin{array}{l}1.19 \\
1.03 \\
1.10\end{array}$ & $\begin{array}{l}0.68 \\
0.66 \\
0.67\end{array}$ \\
\hline $\mathrm{H}^{+}+\mathrm{NH}_{4}^{+}$ & $\mathrm{SO}_{4}^{2-}+\left(\mathrm{NO}_{3}^{-}+\mathrm{NO}_{2}^{-}\right)$ & $\begin{array}{c}1 \\
2 \\
1 \& 2\end{array}$ & $\begin{array}{r}-10.70 \\
4.93 \\
-1.68\end{array}$ & $\begin{array}{l}0.63 \\
0.58 \\
0.60\end{array}$ & $\begin{array}{l}0.82 \\
0.81 \\
0.81\end{array}$ \\
\hline
\end{tabular}




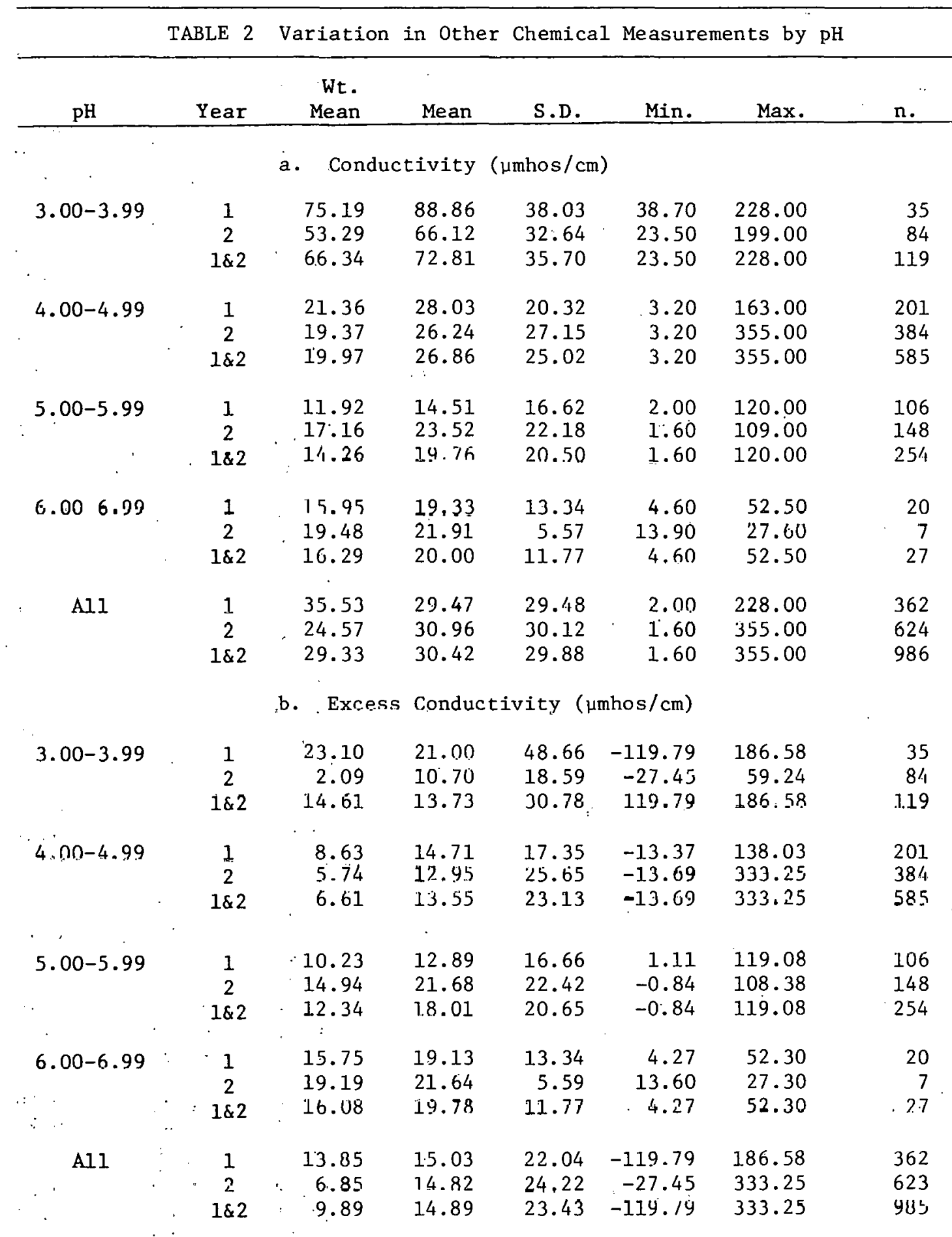


TABLE 2 (continued) $\mathrm{pH}$

\begin{tabular}{|c|c|c|c|c|c|c|c|}
\hline $\mathrm{pH}$ & Year & $\begin{array}{l}\text { Wt. } \\
\text { Mean }\end{array}$ & Mean & S.D. & Min. & Max. & n. \\
\hline \multicolumn{8}{|c|}{ Sulfate Concentration ( $\mu$ eq/ $/ \ell$ ) } \\
\hline $3.00-3.99$ & $\begin{array}{c}1 \\
2 \\
1 \& 2\end{array}$ & $\begin{array}{r}163.23 \\
118.80 \\
146.24\end{array}$ & $\begin{array}{l}223.18 \\
161.57 \\
185.16\end{array}$ & $\begin{array}{r}123.13 \\
92.29 \\
108.93\end{array}$ & $\begin{array}{l}56.20 \\
20.80 \\
20.80\end{array}$ & $\begin{array}{l}562.10 \\
420.60 \\
562.10\end{array}$ & $\begin{array}{r}49 \\
79 \\
128\end{array}$ \\
\hline $4.00-4.99$ & $\begin{array}{c}1 \\
2 \\
1 \& 2\end{array}$ & $\begin{array}{l}60.96 \\
47.23 \\
52.00\end{array}$ & $\begin{array}{l}77.47 \\
61.17 \\
67.81\end{array}$ & $\begin{array}{l}45.69 \\
45.51 \\
46.24\end{array}$ & $\begin{array}{r}41.60 \\
4.20 \\
4.20\end{array}$ & $\begin{array}{l}305.40 \\
308.10 \\
308.10\end{array}$ & $\begin{array}{l}228 \\
332 \\
560\end{array}$ \\
\hline $5.00-5.99$ & $\begin{array}{c}1 \\
2 \\
1 \& 2\end{array}$ & $\begin{array}{l}48.21 \\
31.85 \\
42.12\end{array}$ & $\begin{array}{l}58.91 \\
47.43 \\
53.43\end{array}$ & $\begin{array}{l}34.99 \\
36.90 \\
36.30\end{array}$ & $\begin{array}{r}23.10 \\
4.20 \\
4.20\end{array}$ & $\begin{array}{l}318.50 \\
285.20 \\
318.50\end{array}$ & $\begin{array}{l}127 \\
116 \\
243\end{array}$ \\
\hline $6.00-6.99$ & $\begin{array}{c}1 \\
2 \\
1 \& 2\end{array}$ & $\begin{array}{l}52.61 \\
60.26 \\
53.03\end{array}$ & $\begin{array}{l}74.63 \\
74.45 \\
74.61\end{array}$ & $\begin{array}{l}57.31 \\
32.63 \\
54.16\end{array}$ & $\begin{array}{l}41.60 \\
31.20 \\
31.20\end{array}$ & $\begin{array}{l}287.30 \\
119.30 \\
287.30\end{array}$ & $\begin{array}{r}36 \\
6 \\
42\end{array}$ \\
\hline All & $\begin{array}{c}1 \\
2 \\
1 \& 2\end{array}$ & $\begin{array}{l}90.19 \\
59.17 \\
74.13\end{array}$ & $\begin{array}{l}88.11 \\
77.67 \\
82.12\end{array}$ & $\begin{array}{l}75.54 \\
73.99 \\
74.79\end{array}$ & $\begin{array}{r}23.10 \\
4.20 \\
4.20\end{array}$ & $\begin{array}{l}562.10 \\
674.60 \\
674.60\end{array}$ & $\begin{array}{r}440 \\
594 \\
1034\end{array}$ \\
\hline & & d: $\quad$ Ex & ess Sulf & te Conce & ation & $(\mu$ eq $/ \ell)$ & \\
\hline $3.00-3.99$ & $\begin{array}{c}1 \\
2 \\
1 \& 2\end{array}$ & $\begin{array}{l}175: 46 \\
115.68 \\
137.08\end{array}$ & $\begin{array}{l}227.76 \\
159.22 \\
185.01\end{array}$ & $\begin{array}{r}118.29 \\
88.90 \\
105.79\end{array}$ & $\begin{array}{l}57.82 \\
18.59 \\
18.59\end{array}$ & $\begin{array}{l}561.21 \\
417.13 \\
561.21\end{array}$ & $\begin{array}{r}38 \\
63 \\
101\end{array}$ \\
\hline $4.00-4.99$ & $\begin{array}{c}1 \\
2 \\
1 \& 2\end{array}$ & $\begin{array}{l}57.52 \\
47.59 \\
51.36\end{array}$ & $\begin{array}{l}71.60 \\
53.81 \\
61.55\end{array}$ & $\begin{array}{l}43.47 \\
36.76 \\
40.74\end{array}$ & $\begin{array}{r}17.29 \\
4.18 \\
4.18\end{array}$ & $\begin{array}{l}297.20 \\
199.35 \\
297.20\end{array}$ & $\begin{array}{l}191 \\
248 \\
439\end{array}$ \\
\hline $5.00-5.99$ & $\begin{array}{c}1 \\
2 \\
1 \& 2\end{array}$ & $\begin{array}{l}43.44 \\
23.35 \\
36.02\end{array}$ & $\begin{array}{l}53.18 \\
33.15 \\
43.94\end{array}$ & $\begin{array}{l}28.70 \\
33.06 \\
32.29\end{array}$ & $\begin{array}{r}10.91 \\
-16.02 \\
-16.02\end{array}$ & $\begin{array}{l}183.53 \\
280.41 \\
280.41\end{array}$ & $\begin{array}{r}97 \\
83 \\
180\end{array}$ \\
\hline $6.00-6.99$ & $\begin{array}{c}1 \\
2 \\
1 \& 2\end{array}$ & $\begin{array}{l}43.12 \\
54.14 \\
43.73\end{array}$ & $\begin{array}{l}60.53 \\
68.68 \\
61.73\end{array}$ & $\begin{array}{l}37.30 \\
37.33 \\
36.85\end{array}$ & $\begin{array}{l}10.23 \\
25.94 \\
10.23\end{array}$ & $\begin{array}{l}156.83 \\
117.51 \\
156.83\end{array}$ & $\begin{array}{r}29 \\
5 \\
34\end{array}$ \\
\hline All & $\begin{array}{c}1 \\
2 \\
1 \& 2\end{array}$ & $\begin{array}{l}70.29 \\
58.88 \\
63.94\end{array}$ & $\begin{array}{l}82.38 \\
67.08 \\
74.14\end{array}$ & $\begin{array}{l}73.58 \\
63.58 \\
68.76\end{array}$ & $\begin{array}{r}10.23 \\
-16.02 \\
-16.02\end{array}$ & $\begin{array}{l}561.21 \\
417.13 \\
561.21\end{array}$ & $\begin{array}{l}355 \\
414 \\
769\end{array}$ \\
\hline
\end{tabular}


TABLE 2 (continued) $\mathrm{pH}$

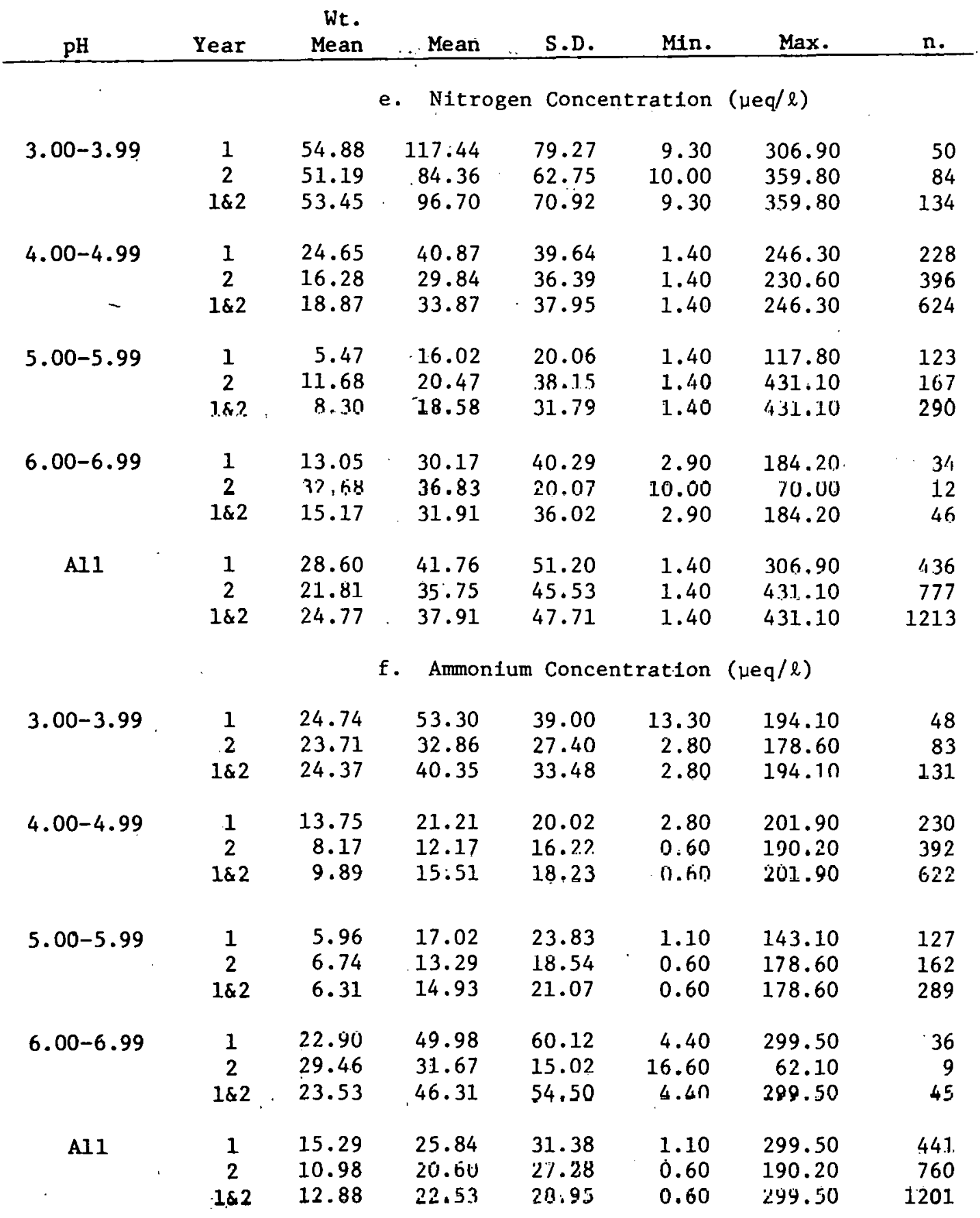


TABLE 2 (continued) $\mathrm{pH}$

\begin{tabular}{|c|c|c|c|c|c|c|c|}
\hline $\mathrm{pH}$ & Year & $\begin{array}{r}\text { Wt. } \\
\text { Mean }\end{array}$ & Mean, & S.D. & Min. & Max. & n. \\
\hline & \multicolumn{7}{|c|}{ g. - Sodium Concentration ( $\mu$ eq/ $\ell$ ) } \\
\hline $3.00-3.99$ & $\begin{array}{c}1 \\
2 \\
1 \& 2\end{array}$ & $\begin{array}{l}13.32 \\
28.72 \\
19.20\end{array}$ & $\begin{array}{l}47.30 \\
41.48 \\
43.74\end{array}$ & $\begin{array}{r}128.65 \\
69.41 \\
96.35\end{array}$ & $\begin{array}{r}2.60 \\
1.70 \\
1.70\end{array}$ & $\begin{array}{l}891.30 \\
434.80 \\
891.30\end{array}$ & $\begin{array}{r}47 \\
74 \\
121\end{array}$ \\
\hline $4.00-4.99$ & $\begin{array}{c}1 \\
2 \\
1 \& 2\end{array}$ & $\begin{array}{l}33.25 \\
46.46 \\
42.29\end{array}$ & $\begin{array}{l}47.00 \\
78.19 \\
66.24\end{array}$ & $\begin{array}{r}79.20 \\
205.04 \\
168.90\end{array}$ & $\begin{array}{l}0.40 \\
0.40 \\
0.40\end{array}$ & $\begin{array}{r}669.60 \\
2543.60 \\
2543.60\end{array}$ & $\begin{array}{l}220 \\
354 \\
574\end{array}$ \\
\hline $5.00-5.99$ & $\begin{array}{c}1 \\
2 \\
1 \& 2\end{array}$ & $\begin{array}{l}37.58 \\
78.30 \\
55.35\end{array}$ & $\begin{array}{r}38.73 \\
103.85 \\
72.72\end{array}$ & $\begin{array}{r}88.97 \\
146.50 \\
126.45\end{array}$ & $\begin{array}{l}0.90 \\
0.40 \\
0.40\end{array}$ & $\begin{array}{l}765.20 \\
730.50 \\
765.20\end{array}$ & $\begin{array}{l}120 \\
131 \\
251\end{array}$ \\
\hline $6.00-6.99$ & $\begin{array}{c}1 \\
2 \\
1 \& 2\end{array}$ & $\begin{array}{l}66.15 \\
41.66 \\
64.21\end{array}$ & $\begin{array}{l}78.53 \\
37.82 \\
71.75\end{array}$ & $\begin{array}{r}197.31 \\
16.99 \\
180.37\end{array}$ & $\begin{array}{r}3.90 \\
14.80 \\
3.90\end{array}$ & $\begin{array}{r}1078.30 \\
61.70 \\
1078.30\end{array}$ & $\begin{array}{r}30 \\
6 \\
36\end{array}$ \\
\hline All & $\begin{array}{c}1 \\
2 \\
1 \& 2\end{array}$ & $\begin{array}{l}28.85 \\
49.62 \\
40.25\end{array}$ & $\begin{array}{l}46.92 \\
78.74 \\
65.55\end{array}$ & $\begin{array}{l}101.01 \\
176.84 \\
150.88\end{array}$ & $\begin{array}{l}0.40 \\
0.40 \\
0.40\end{array}$ & $\begin{array}{l}1078.30 \\
2543.60 \\
2543.60\end{array}$ & $\begin{array}{r}417 \\
589 \\
1006\end{array}$ \\
\hline & & & Ch1 & $\operatorname{Con}$ & ation & $\mu e q / \ell)$ & \\
\hline $3.00-3.99$ & $\begin{array}{c}1 \\
2 \\
1 \& 2\end{array}$ & $\begin{array}{l}23.87 \\
27.33 \\
25.20\end{array}$ & $\begin{array}{l}69.82 \\
45.55 \\
54.58\end{array}$ & $\begin{array}{r}197.89 \\
60.25 \\
.129 .56\end{array}$ & $\begin{array}{l}5.60 \\
5.60 \\
5.60\end{array}$ & $\begin{array}{r}1396.40 \\
378.00 \\
1396.40\end{array}$ & $\begin{array}{r}48 \\
81 \\
129\end{array}$ \\
\hline $4.00-4.99$ & $\begin{array}{c}1 \\
2 \\
1 \& 2\end{array}$ & $\begin{array}{l}49.80 \\
50.51 \\
50.29\end{array}$ & $\begin{array}{l}74.12 \\
73.73 \\
73.88\end{array}$ & $\begin{array}{l}143.11 \\
126.07 \\
132.60\end{array}$ & $\begin{array}{l}5.60 \\
5.60 \\
5.60\end{array}$ & $\begin{array}{l}1317.40 \\
1458.50 \\
1458.50\end{array}$ & $\begin{array}{l}226 \\
376 \\
602\end{array}$ \\
\hline $5.00-5.99$ & $\begin{array}{c}1 \\
2 \\
1 \& 2\end{array}$ & $\begin{array}{l}55.87 \\
87.51 \\
69.98\end{array}$ & $\begin{array}{r}66.73 \\
111.31 \\
90.64\end{array}$ & $\begin{array}{l}115.12 \\
158.14 \\
141.36\end{array}$ & $\begin{array}{l}5.60 \\
5.60 \\
5.60\end{array}$ & $\begin{array}{l}894.30 \\
860.40 \\
894.30\end{array}$ & $\begin{array}{l}121 \\
140 \\
261\end{array}$ \\
\hline $6.00-6.99$ & $\begin{array}{c}1 \\
2 \\
1 \& 2\end{array}$ & $\begin{array}{l}91.11 \\
35.40 \\
88.02\end{array}$ & $\begin{array}{r}111.55 \\
37.60 \\
100.73\end{array}$ & $\begin{array}{r}234.65 \\
14.25 \\
218.01\end{array}$ & $\begin{array}{r}5.60 \\
19.70 \\
5.60\end{array}$ & $\begin{array}{r}1311.80 \\
56.40 \\
1311.80\end{array}$ & $\begin{array}{r}35 \\
6 \\
41\end{array}$ \\
\hline All & $\begin{array}{c}1 \\
2 \\
1 \& 2\end{array}$ & $\begin{array}{l}44.23 \\
54.48 \\
49.94\end{array}$ & $\begin{array}{l}74.87 \\
82.63 \\
79.58\end{array}$ & $\begin{array}{l}152.35 \\
132.07 \\
140.37\end{array}$ & $\begin{array}{l}5.60 \\
5.60 \\
5.60\end{array}$ & $\begin{array}{l}1396.40 \\
1458.50 \\
1458.50\end{array}$ & $\begin{array}{r}431 \\
666 \\
1097\end{array}$ \\
\hline
\end{tabular}


TABLE 2 (continued) $\mathrm{pH}$

\begin{tabular}{|c|c|c|c|c|c|c|c|}
\hline $\mathrm{pH}$ & Year & $\begin{array}{l}\text { Wt. } \\
\text { Mean }\end{array}$ & Mean & S.D. & Min. & Max. & n. \\
\hline \multirow{4}{*}{$3.00-3.99$} & & i. Exces & Chloride & \multicolumn{4}{|c|}{ Concentration (ueq/l) } \\
\hline & 1 & 8.23 & 20.19 & 63.31 & -29.26 & 353.58 & 35 \\
\hline & 2 & -13.87 & -8.41 & 33.68 & -178.62 & 37.58 & 44 \\
\hline & $1 \& 2$ & -6.39 & 4.26 & 50.76 & -1.78 .62 & 353.58 & 79 \\
\hline \multirow[t]{3}{*}{$4.00-4.99$} & 1 & 13.68 & 15.97 & 49.05 & -151.67 & 329.07 & 144 \\
\hline & 2 & -6.13 & -12.26 & 60.42 & -360.99 & 265.18 & 205 \\
\hline & $1 \& 2$ & 0.31 & -0.62 & 57.64 & -360.99 & 329.07 & 349 \\
\hline \multirow[t]{3}{*}{$5.00-5.99$} & 1 & 14.71 & 19.61 & 28.10 & -68.14 & 133.72 & 66 \\
\hline & 2 & 10.61 & -8.32 & 18.00 & $-296,37$ & 232.64 & 93 \\
\hline & $1 \& 2$ & 0.91 & 3.28 & 43.10 & -296.37 & 232.64 & 159 \\
\hline \multirow[t]{3}{*}{$6.00-6.99$} & 1 & 12.61 & 16.50 & 18.69 & -4.26 & 64.35 & 21 \\
\hline & 2 & -13.00 & -8.89 & 18.01 & -31.20 & 10.88 & 3 \\
\hline & $1 \notin 2$ & 10.86 & 11.62 & 20.87 & -31.20 & 64.35 & 26 \\
\hline \multirow[t]{4}{*}{ All } & 1 & 13.29 & 17.47 & 45.12 & -151.67 & 353.58 & 266 \\
\hline & 2 & -7.80 & -10.01 & 54.73 & -360.99 & 265.18 & 360 \\
\hline & $1 \& 2$ & 0.04 & 1.66 & 52.62 & -360.99 & 353.58 & 626 \\
\hline & & j. Nitro & n/Sulfate & Ratio & & & \\
\hline \multirow[t]{3}{*}{$3.00-3.99$} & 1 & 0.35 & 0.65 & 0.67 & 0.04 & 3.59 & 48 \\
\hline & 2 & 0.44 & 0.59 & 0.52 & 0.08 & 4.08 & 79 \\
\hline & $1 \& 2$ & 0.38 & 0.61 & 0.58 & 0.04 & 4.08 & 127 \\
\hline \multirow[t]{3}{*}{$4.00-4.99$} & 1 & 0.41 & 0.54 & 0.41 & 0.10 & 2.48 & 212 \\
\hline & 2 & 0.40 & 0.49 & 0.38 & 0.10 & 2.53 & 276 \\
\hline & $1 \& 2$ & 0.40 & 0.51 & 0.40 & 0.10 & 2.53 & 488 \\
\hline \multirow{3}{*}{$5.00-5.99$} & 1 & 0.23 & 0.33 & 0.24 & 0.06 & 1.31 & 88 \\
\hline & 2 & 0.34 & 0.41 & 0.37 & 0.05 & 1.90 & 80 \\
\hline & $1 \& 2$ & 0.27 & 0.37 & 0.31 & 0.05 & 1.90 & 168 \\
\hline \multirow[t]{3}{*}{$6.00-6.99$} & 1 & 0.25 & 0.36 & 0.22 & 0.10 & 0.99 & 29 \\
\hline & 2 & 0.83 & 0.72 & 0.49 & 0.37 & 1.68 & 6 \\
\hline & $1 \& 2$ & 0.29 & 0.42 & 0.31 & 0.10 & 1.68 & 35 \\
\hline \multirow[t]{3}{*}{ All } & 1 & $\quad 0.35$ & 0.49 & 0.43 & 0.04 & 3.59 & 377 \\
\hline & 2 & 0.42 & 0.50 & 0.41 & 0.05 & 4.08 & 491 \\
\hline & $1 \& 2$ & 0.38 & 0.50 & 0.42 & 0.04 & 4.08 & $B 68$ \\
\hline
\end{tabular}


TABLE 2 (continued) pH

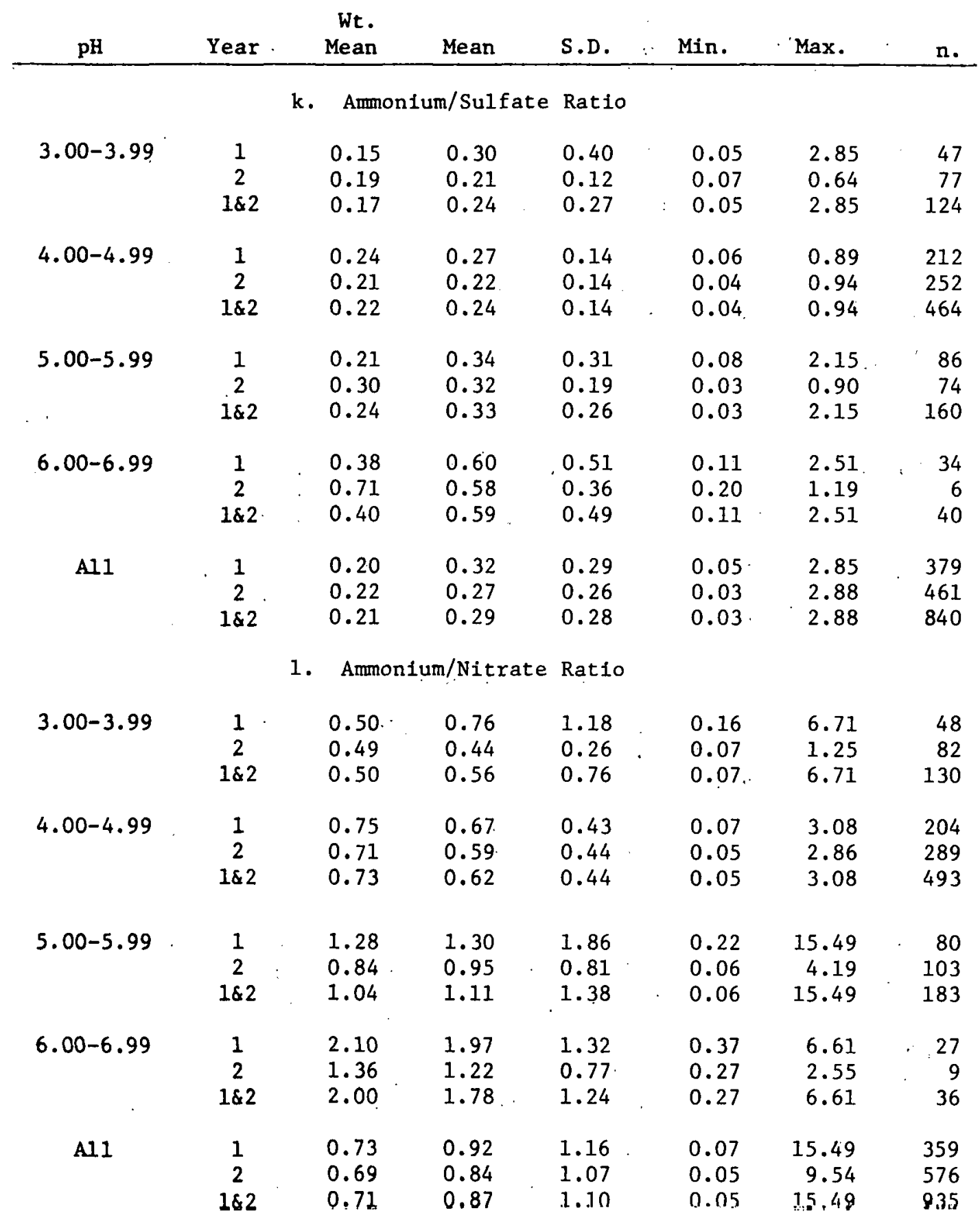


TABLE 2 (continued) $\mathrm{pH}$

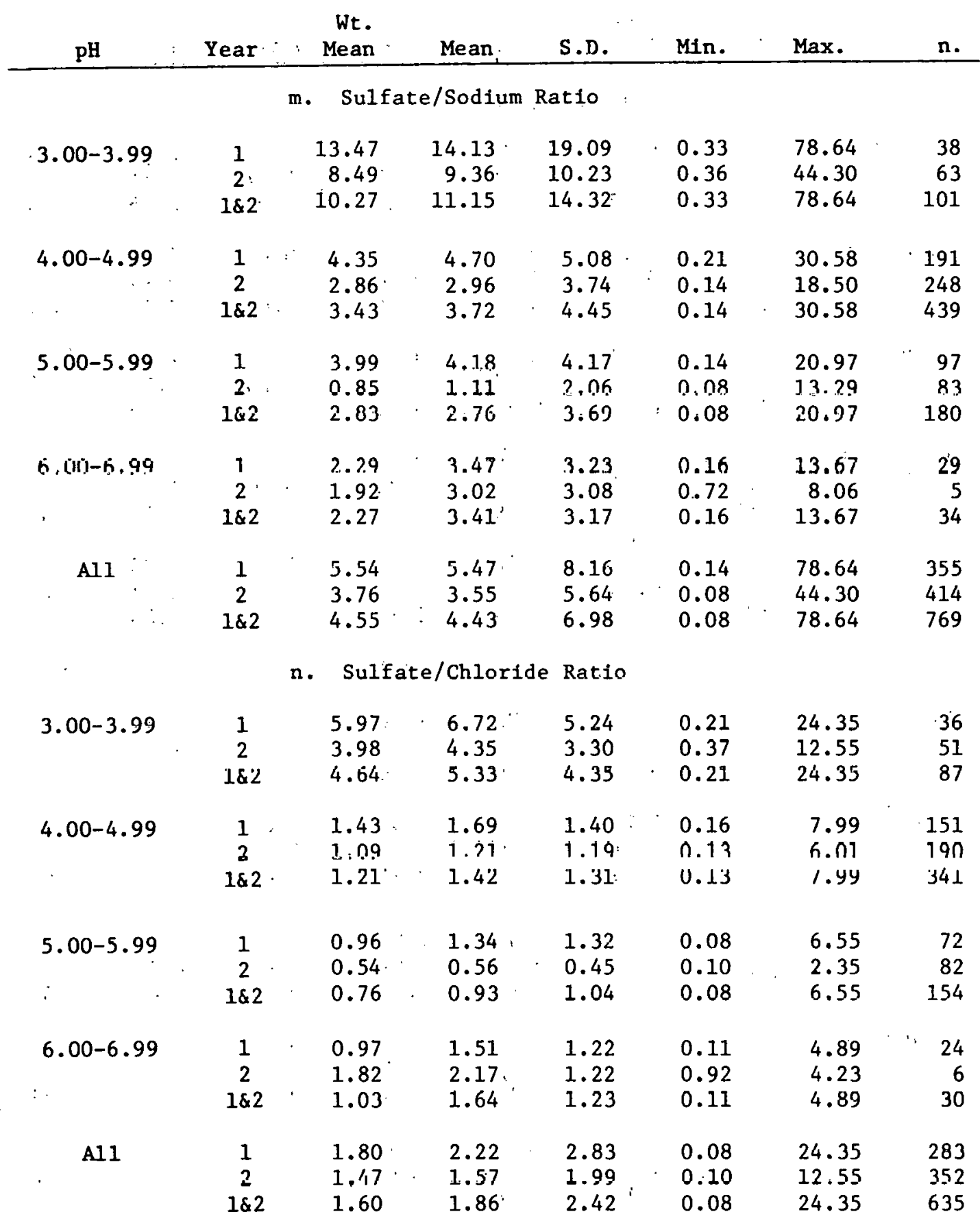


TABLE 2 (continued) $\mathrm{pH}$

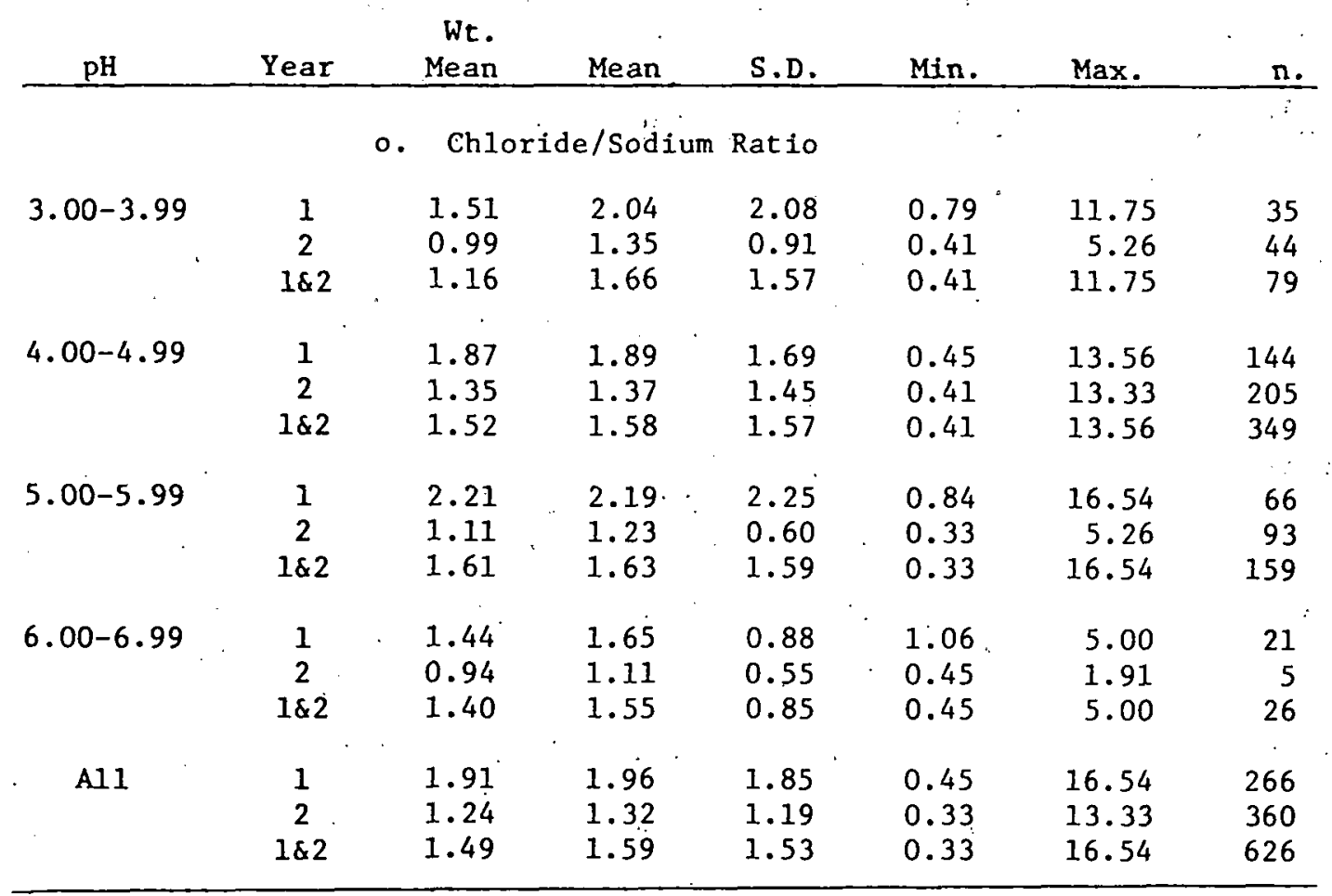




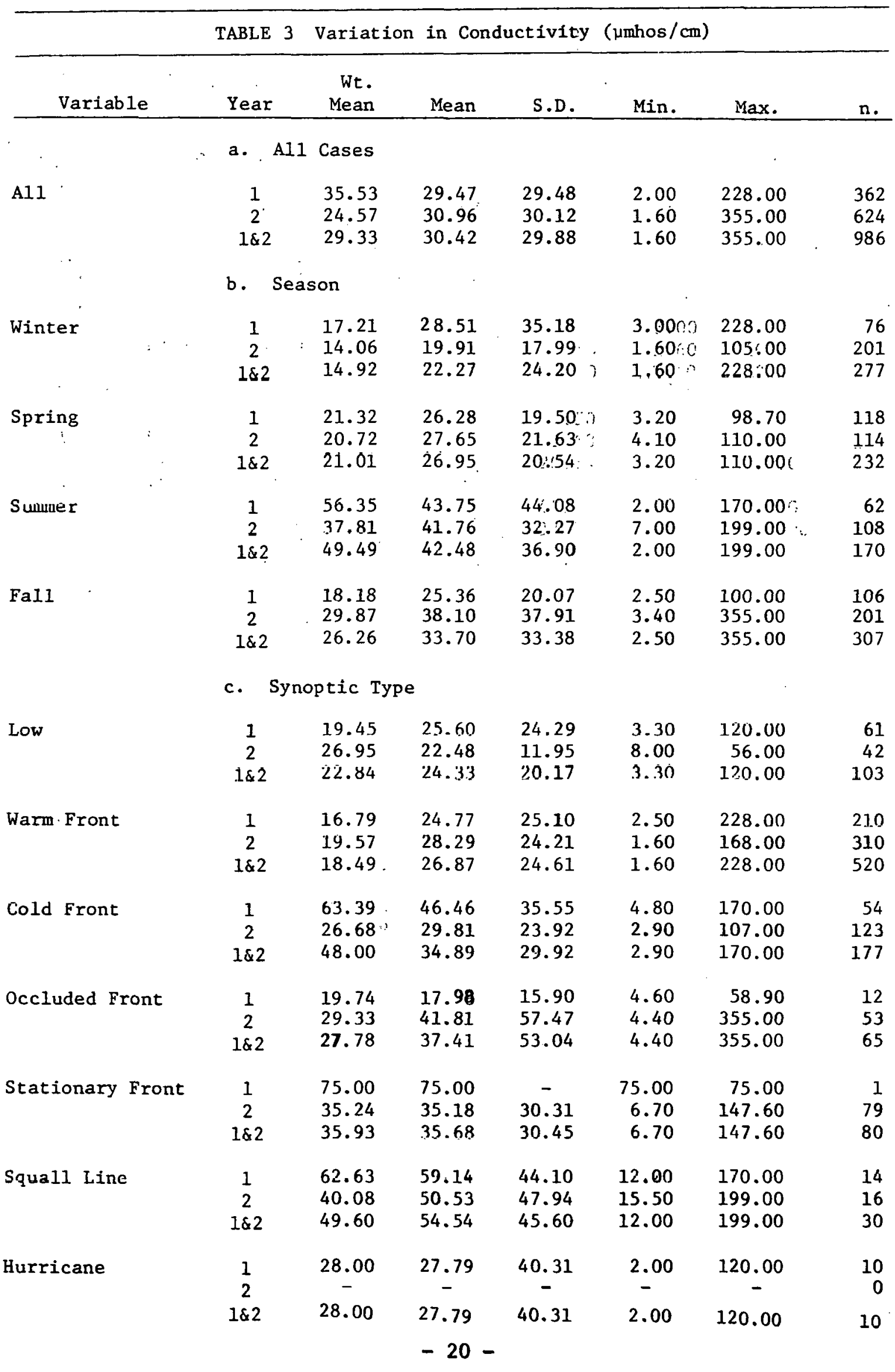


TABLE 3 (continued) Conductivity

Wt .

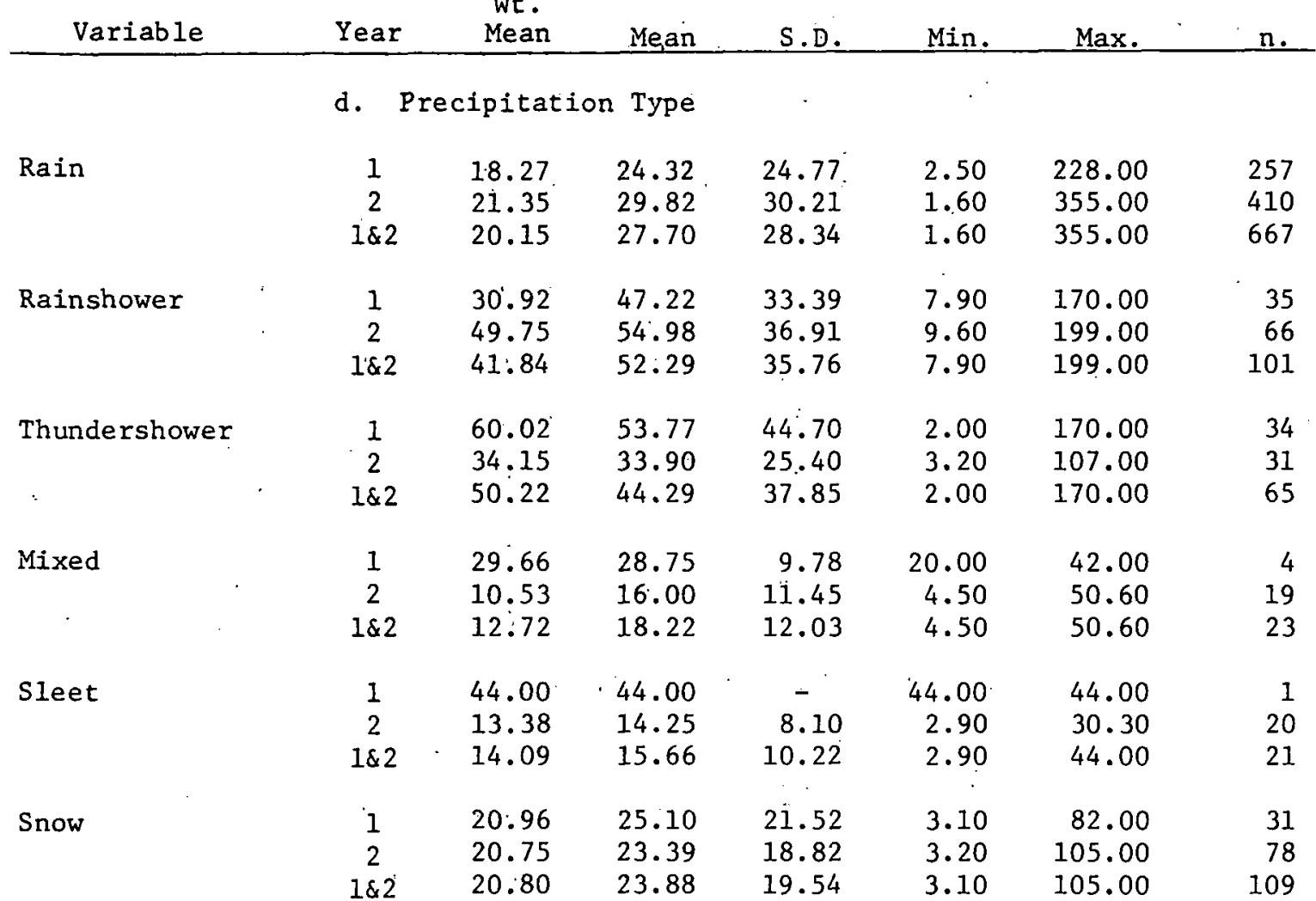

e: Precipitation Rate $(\mathrm{mm} / \mathrm{hr})$

\begin{tabular}{|c|c|c|c|c|c|c|c|}
\hline $0.0-1.0$ & $\begin{array}{c}1 \\
2 \\
1 \& 2\end{array}$ & $\begin{array}{l}34.99 \\
38.93 \\
37.52\end{array}$ & $\begin{array}{l}35.09 \\
38.87 \\
37.54\end{array}$ & $\begin{array}{l}30.40 \\
36.31 \\
34.36\end{array}$ & $\begin{array}{l}3.10 \\
3.10 \\
3.10\end{array}$ & $\begin{array}{l}170.00 \\
355.00 \\
355.00\end{array}$ & $\begin{array}{l}158 \\
290 \\
448\end{array}$ \\
\hline $1.1-2.5$ & $\begin{array}{c}1 \\
2 \\
1 \& 2\end{array}$ & $\begin{array}{l}27.02 \\
27.41 \\
27.26\end{array}$ & $\begin{array}{l}28.61 \\
28.36 \\
28.46\end{array}$ & $\begin{array}{l}30.56 \\
24.18 \\
26.83\end{array}$ & $\begin{array}{l}2.00 \\
2.90 \\
2.00\end{array}$ & $\begin{array}{l}228.00 \\
137.30 \\
228.00\end{array}$ & $\begin{array}{l}113 \\
174 \\
287\end{array}$ \\
\hline $2.6-6.0$ & $\begin{array}{c}1 \\
2 \\
1 \& 2\end{array}$ & $\begin{array}{l}17.06 \\
20.11 \\
19.11\end{array}$ & $\begin{array}{l}17.63 \\
20.23 \\
19.35\end{array}$ & $\begin{array}{l}16.69 \\
16.38 \\
16.48\end{array}$ & $\begin{array}{l}3.60 \\
1.60 \\
1.60\end{array}$ & $\begin{array}{r}100.00 \\
86.60 \\
100.00\end{array}$ & $\begin{array}{r}56 \\
110 \\
166\end{array}$ \\
\hline $6.1-12.0$ & $\begin{array}{c}1 \\
2 \\
1 \& 2\end{array}$ & $\begin{array}{l}50.42 \\
15.40 \\
35.92\end{array}$ & $\begin{array}{l}25.03 \\
15.05 \\
18.92\end{array}$ & $\begin{array}{l}33.22 \\
12.09 \\
23.03\end{array}$ & $\begin{array}{l}2.50 \\
2.70 \\
2.50\end{array}$ & $\begin{array}{r}120.00 \\
53.30 \\
120.00\end{array}$ & $\begin{array}{l}26 \\
41 \\
67\end{array}$ \\
\hline$>12.0$ & $\begin{array}{c}1 \\
2 \\
1 \& 2\end{array}$ & $\begin{array}{l}27.99 \\
32.64 \\
30.58\end{array}$ & $\begin{array}{l}28.04 \\
30.43 \\
29.24\end{array}$ & $\begin{array}{l}31.08 \\
26.19 \\
27.91\end{array}$ & $\begin{array}{l}3.00 \\
4.90 \\
3.00\end{array}$ & $\begin{array}{l}80.00 \\
80.50 \\
80.50\end{array}$ & $\begin{array}{r}9 \\
9 \\
18\end{array}$ \\
\hline
\end{tabular}


TABLE 3 (continued) Conductivity

Wt.

\begin{tabular}{|c|c|c|c|c|c|c|c|}
\hline Variable & Year & Mean & Mean & S.D. & Min. & Max. & n. \\
\hline & f. & Speed & (m $\sec ^{-}$ & at 108 & & & \\
\hline $0-2$ & $\begin{array}{c}1 \\
2 \\
1 \& 2\end{array}$ & $\begin{array}{l}18.40 \\
29.12 \\
22.45\end{array}$ & $\begin{array}{l}26.87 \\
36.62 \\
31.87\end{array}$ & $\begin{array}{l}18.85 \\
24.49 \\
22.18\end{array}$ & $\begin{array}{r}9.70 \\
11.30 \\
9.70\end{array}$ & $\begin{array}{l}69.50 \\
96.60 \\
96.60\end{array}$ & $\begin{array}{l}18 \\
19 \\
37\end{array}$ \\
\hline $3-5$ & $\begin{array}{c}1 \\
2 \\
182\end{array}$ & $\begin{array}{l}50.01 \\
31.90 \\
44.55\end{array}$ & $\begin{array}{l}31.10 \\
35.41 \\
33.47\end{array}$ & $\begin{array}{l}31.21 \\
30.98 \\
31.11\end{array}$ & $\begin{array}{l}2.00 \\
1.60 \\
1.60\end{array}$ & $\begin{array}{l}170.00 \\
199.00 \\
199.00\end{array}$ & $\begin{array}{l}134 \\
164 \\
298\end{array}$ \\
\hline $6-9$ & $\begin{array}{c}1 \\
2 \\
182\end{array}$ & $\begin{array}{l}28.48 \\
26.46 \\
26.97\end{array}$ & $\begin{array}{l}30.95 \\
31.18 \\
31.1 \cap\end{array}$ & $\begin{array}{l}32.07 \\
33.65 \\
33.12\end{array}$ & $\begin{array}{l}3.10 \\
2.70 \\
2.7 n\end{array}$ & $\begin{array}{l}228.00 \\
355.00 \\
355 . \mathrm{n} n\end{array}$ & $\begin{array}{l}131 \\
282 \\
413\end{array}$ \\
\hline$\geq 10$ & $\begin{array}{c}1 \\
\dot{2} \\
1 \& 2\end{array}$ & $\begin{array}{l}17.01 \\
14.28 \\
18.35\end{array}$ & $\begin{array}{l}24.85 \\
24.84 \\
26.12\end{array}$ & $\begin{array}{l}23.23 \\
27.15 \\
22.45\end{array}$ & $\begin{array}{l}3.00 \\
2.911 \\
2.90\end{array}$ & $\begin{array}{l}120.00 \\
1 \text { ถิ. } \\
120.00\end{array}$ & $\begin{array}{r}79 \\
140 \\
219\end{array}$ \\
\hline
\end{tabular}

g. Wind Direction at $108 \mathrm{~m}$

\begin{tabular}{|c|c|c|c|c|c|c|c|c|c|}
\hline $\mathbf{N}$ & & & $\begin{array}{c}1 \\
2 \\
1 \& 2\end{array}$ & $\begin{array}{l}19.05 \\
26.29 \\
22.13\end{array}$ & $\begin{array}{l}21.66 \\
28.00 \\
25.03\end{array}$ & $\begin{array}{l}13.23 \\
17.18 \\
15.66\end{array}$ & $\begin{array}{l}4.70 \\
8.50 \\
4.70\end{array}$ & $\begin{array}{r}54.00 \\
107.00 \\
107.00\end{array}$ & $\begin{array}{l}29 \\
33 \\
62\end{array}$ \\
\hline $\mathrm{NE}$ & & & $\begin{array}{c}1 \\
2 \\
1 \& 2\end{array}$ & $\begin{array}{l}15.24 \\
18.01 \\
17.59\end{array}$ & $\begin{array}{l}23.52 \\
23.49 \\
23.50\end{array}$ & $\begin{array}{l}21.49 \\
20.79 \\
20.88\end{array}$ & $\begin{array}{l}2.00 \\
1.60 \\
1.60\end{array}$ & $\begin{array}{l}100.00 \\
107.00 \\
107.00\end{array}$ & $\begin{array}{r}47 \\
210 \\
257\end{array}$ \\
\hline$E$ & & & $\begin{array}{c}1 \\
2 \\
1 \& 2\end{array}$ & $\begin{array}{l}19.22 \\
27.02 \\
23.38\end{array}$ & $\begin{array}{l}25.21 \\
33.67 \\
30.72\end{array}$ & $\begin{array}{l}23.06 \\
24.61 \\
24.36\end{array}$ & $\begin{array}{l}3.10 \\
6.20 \\
3.10\end{array}$ & $\begin{array}{l}120.00 \\
128.30 \\
128.30\end{array}$ & $\begin{array}{r}70 \\
131 \\
201\end{array}$ \\
\hline SE & & · & $\begin{array}{c}\frac{1}{2} \\
1 \& 2\end{array}$ & $\begin{array}{l}49.20 \\
28.31 \\
42.22\end{array}$ & $\begin{array}{r}35.42 \\
42.84 \\
39.45\end{array}$ & $\begin{array}{l}39.55 \\
38.88 \\
39.22\end{array}$ & $\begin{array}{l}2.50 \\
4.50 \\
2.50\end{array}$ & $\begin{array}{l}228.00 \\
235.00 \\
235.00\end{array}$ & $\begin{array}{r}64 \\
76 \\
140\end{array}$ \\
\hline $\mathbf{S}$ & '. & $\begin{array}{r}. \\
. \\
.\end{array}$ & $\begin{array}{c}1 \\
2 \\
1 \& 2\end{array}$ & $\begin{array}{l}20.53 \\
17.58 \\
18.49\end{array}$ & $\begin{array}{l}28.37 \\
30.83 \\
29.86\end{array}$ & $\begin{array}{l}29.30 \\
45.44 \\
39.76\end{array}$ & $\begin{array}{l}4.80 \\
2.90 \\
2.90\end{array}$ & $\begin{array}{l}170.00 \\
355.00 \\
355.00\end{array}$ & $\begin{array}{r}54 \\
83 \\
137\end{array}$ \\
\hline SW & & & $\begin{array}{c}1 \\
2 \\
1 \& 2\end{array}$ & $\begin{array}{l}39.73 \\
35.67 \\
36.83\end{array}$ & $\begin{array}{l}44.32 \\
31.04 \\
38.25\end{array}$ & $\begin{array}{l}34.38 \\
25.45 \\
29.71\end{array}$ & $\begin{array}{l}3.30 \\
3.40 \\
3.30\end{array}$ & $\begin{array}{l}170.00 \\
1 \cap 6, n \\
170.00\end{array}$ & $\begin{array}{r}43 \\
6 ? \\
105\end{array}$ \\
\hline W & & '. & $\begin{array}{c}1 \\
2 \\
1 \& 2\end{array}$ & $\begin{array}{l}48.99 \\
51.61 \\
50.70\end{array}$ & $\begin{array}{l}31.41 \\
44.26 \\
38.42\end{array}$ & $\begin{array}{l}31.79 \\
44.23 \\
39.04\end{array}$ & $\begin{array}{l}7.60 \\
4.80 \\
4.80\end{array}$ & $\begin{array}{l}120.00 \\
199.00 \\
199.00\end{array}$ & $\begin{array}{l}15 \\
18 \\
33\end{array}$ \\
\hline NW & $\cdot$ & & $\begin{array}{c}\frac{1}{2} \\
1 \& 2\end{array}$ & $\begin{array}{l}49.02 \\
28.20 \\
45.02\end{array}$ & $\begin{array}{l}24.86 \\
30.04 \\
25.97\end{array}$ & $\begin{array}{l}25.68 \\
19.34 \\
24.37\end{array}$ & $\begin{array}{l}3.10 \\
9.00 \\
3.10\end{array}$ & $\begin{array}{r}120.00 \\
57.40 \\
120.00\end{array}$ & $\begin{array}{l}40 \\
11 \\
51\end{array}$ \\
\hline
\end{tabular}


TABLE 3 (continued) Conductivity

\begin{tabular}{|c|c|c|c|c|c|c|c|}
\hline Variable & Year & $\begin{array}{l}\text { Wt. } \\
\text { Mean }\end{array}$ & Mean & S.D. & Min. & Max. & n. \\
\hline " & h. & nperatur & $\left({ }^{\circ} \mathrm{C}\right)$ & : & & & \\
\hline-10.0 to -5.1 & $\begin{array}{c}1 \\
2 \\
1 \& 2\end{array}$ & $\begin{array}{l}63.00 \\
77.30: \\
63.09\end{array}$ & $\begin{array}{l}63.00 \\
77.30 \\
70.15\end{array}$ & $\frac{-}{10.11}$ & $\begin{array}{l}63.00 \\
77.30 \\
63.00\end{array}$ & $\begin{array}{l}63.00 \\
77.30 \\
77.30\end{array}$ & $\begin{array}{l}1 \\
1 \\
2\end{array}$ \\
\hline-5.0 to -0.1 & $\begin{array}{c}1 \\
2 \\
1 \& 2\end{array}$ & $\begin{array}{l}27.25 \\
16.58 \\
18.33\end{array}$ & $\begin{array}{l}30.26 \\
18.31 \\
20.82\end{array}$ & $\begin{array}{l}21.31 \\
12.44 \\
15.42\end{array}$ & $\begin{array}{l}8.00 \\
2.90 \\
2.90\end{array}$ & $\begin{array}{l}82.00 \\
70.40 \\
82.00\end{array}$ & $\begin{array}{r}21 \\
79 \\
100\end{array}$ \\
\hline 0.0 to 4.9 & $\begin{array}{c}1 \\
2 \\
1 \& 2\end{array}$ & $\begin{array}{l}19.01 \\
17.21 \\
17.92\end{array}$ & $\begin{array}{l}23.83 \\
21.35 \\
22.24\end{array}$ & $\begin{array}{l}16.38 \\
17.88 \\
17.35\end{array}$ & $\begin{array}{l}3.10 \\
4.50 \\
3.10\end{array}$ & $\begin{array}{r}90.00 \\
105.00 \\
105.00\end{array}$ & $\begin{array}{r}56 \\
100 \\
156 \\
\ldots\end{array}$ \\
\hline 5.0 to 9.9 & $\begin{array}{c}1 \\
2 \\
1 \& 2\end{array}$ & $\begin{array}{l}15.14 \\
21.29 \\
18.78\end{array}$ & $\begin{array}{l}23.25 \\
36.06 \\
30.52\end{array}$ & $\begin{array}{l}26.81 \\
42.44 \\
36.99\end{array}$ & $\begin{array}{l}3.20 \\
1.60 \\
1.60\end{array}$ & $\begin{array}{l}228.00 \\
355.00 \\
355.00\end{array}$ & $\begin{array}{r}96 \\
126 \\
222\end{array}$ \\
\hline 10.0 to 14.9 & $\begin{array}{c}1 \\
2 \\
1 \& 2\end{array}$ & $\begin{array}{l}24.23 \\
20.62 \\
21.59\end{array}$ & $\begin{array}{l}31.12 \\
27.65 \\
28.69\end{array}$ & $\begin{array}{l}27.58 \\
22.22 \\
23.94\end{array}$ & $\begin{array}{l}3.00 \\
2.90 \\
2.90\end{array}$ & $\begin{array}{l}163.00 \\
128.30 \\
163.00\end{array}$ & $\begin{array}{r}75 \\
176 \\
251\end{array}$ \\
\hline 15.0 to 19.9 & $\begin{array}{c}1 \\
2 \\
1 \& 2\end{array}$ & $\begin{array}{l}23.47 \\
30.18 \\
28.08\end{array}$ & $\begin{array}{l}35.59 \\
41.35 \\
39.57\end{array}$ & $\begin{array}{l}30.59 \\
29.86 \\
30.07\end{array}$ & $\begin{array}{l}2.00 \\
3.40 \\
2.00\end{array}$ & $\begin{array}{l}100.00 \\
147.60 \\
147.60\end{array}$ & $\begin{array}{r}34 \\
76 \\
110\end{array}$ \\
\hline 20.0 to 24.9 & $\begin{array}{c}1 \\
2 \\
1 \& 2\end{array}$ & $\begin{array}{l}52.74 \\
41.64 \\
49.44\end{array}$ & $\begin{array}{l}34.93 \\
44.31 \\
39.11\end{array}$ & $\begin{array}{l}38.99 \\
32.42 \\
36.38\end{array}$ & $\begin{array}{l}2.50 \\
8.00 \\
2.50\end{array}$ & $\begin{array}{l}170.00 \\
168.00 \\
170.00\end{array}$ & $\begin{array}{r}77 \\
62 \\
139\end{array}$ \\
\hline 25.0 to 29.9 & $\begin{array}{c}1 \\
2 \\
1 \& 2\end{array}$ & $\begin{array}{l}56.91 \\
49.92 \\
53.81\end{array}$ & $\begin{array}{l}84.70 \\
90.70 \\
88.70\end{array}$ & $\begin{array}{l}49.92 \\
73.47 \\
61.21\end{array}$ & $\begin{array}{l}49.40 \\
43.30 \\
43.30\end{array}$ & $\begin{array}{l}120.00 \\
199.00 \\
199.00\end{array}$ & $\begin{array}{l}2 \\
4 \\
6\end{array}$ \\
\hline
\end{tabular}


TABLE 4 Variation in Excess Conductivity ( $\mu$ mhos $/ \mathrm{cm}$ )

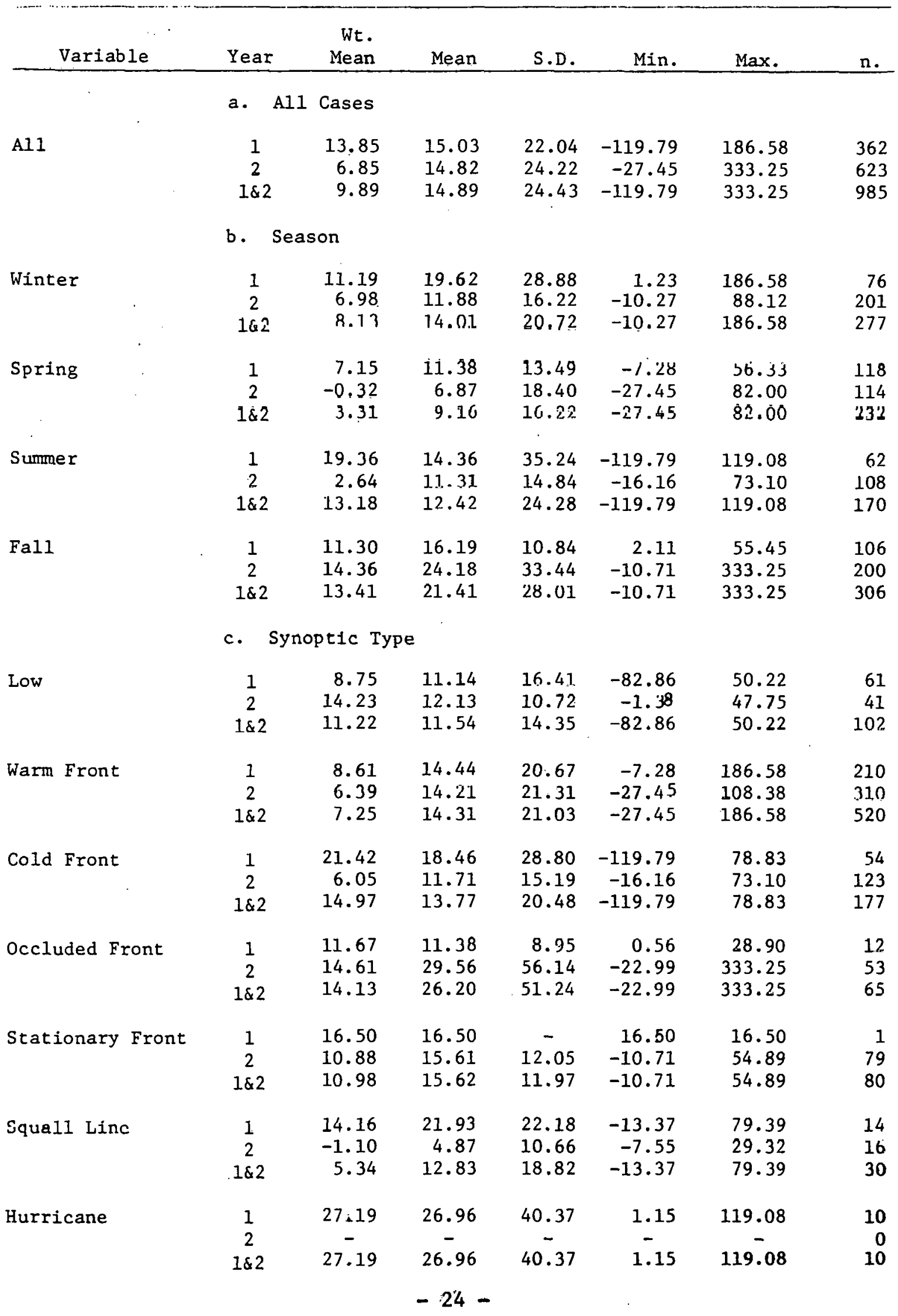


TABLE 4 (continued) Excess Conductivity

\begin{tabular}{|c|c|c|c|c|c|c|c|}
\hline Variable & Year & $\begin{array}{l}\text { Wt. } \\
\text { Mean }\end{array}$ & Mean & S.D. & Min. & Max. & $\mathrm{n}$. \\
\hline & \multicolumn{7}{|c|}{ Precipitation Type } \\
\hline Rain & $\begin{array}{c}1 \\
2 \\
1 \& 2\end{array}$ & $\begin{array}{l}9.05 \\
8.35 \\
8.62\end{array}$ & $\begin{array}{l}14.45 \\
16.85 \\
15.92\end{array}$ & $\begin{array}{l}19.44 \\
27.16 \\
24.48\end{array}$ & $\begin{array}{r}-7.28 \\
-27.45 \\
-27.45\end{array}$ & $\begin{array}{l}186.58 \\
333.25 \\
333.25\end{array}$ & $\begin{array}{l}257 \\
409 \\
666\end{array}$ \\
\hline Rainshower & $\begin{array}{c}1 \\
2 \\
1 \& 2\end{array}$ & $\begin{array}{r}13.84 \\
8.69 \\
10.85\end{array}$ & $\begin{array}{l}23.43 \\
16.75 \\
19.07\end{array}$ & $\begin{array}{l}16.77 \\
18.43 \\
18.07\end{array}$ & $\begin{array}{r}-0.50 \\
-16.16 \\
-16.16\end{array}$ & $\begin{array}{l}79.39 \\
73.10 \\
79.39\end{array}$ & $\begin{array}{r}35 \\
66 \\
101\end{array}$ \\
\hline Thundershower & $\begin{array}{c}1 \\
2 \\
1 \& 2\end{array}$ & $\begin{array}{r}20.33 \\
1.47 \\
13.19\end{array}$ & $\begin{array}{r}10.89 \\
3.94 \\
7.57\end{array}$ & $\begin{array}{l}42.03 \\
12.10 \\
31.49\end{array}$ & $\begin{array}{r}-119.79 \\
-11.13 \\
-119.79\end{array}$ & $\begin{array}{l}83.88 \\
45.74 \\
83.88\end{array}$ & $\begin{array}{l}34 \\
31 \\
65\end{array}$ \\
\hline Mixed & $\begin{array}{c}1 \\
2 \\
1 \& 2\end{array}$ & $\begin{array}{l}6.63 \\
2.33 \\
2.82\end{array}$ & $\begin{array}{l}6.54 \\
6.03 \\
6.12\end{array}$ & $\begin{array}{l}5.35 \\
7.42 \\
7.00\end{array}$ & $\begin{array}{l}-0.82 \\
-4.70 \\
-4.70\end{array}$ & $\begin{array}{l}12.00 \\
28.93 \\
28.93\end{array}$ & $\begin{array}{r}4 \\
19 \\
23\end{array}$ \\
\hline Sleet & $\begin{array}{c}1 \\
2 \\
1 \& 2\end{array}$ & $\begin{array}{r}37.29 \\
2.27 \\
3.09\end{array}$ & $\begin{array}{r}37.29 \\
2.90 \\
4.54\end{array}$ & $\begin{array}{r}- \\
8.30 \\
11.03\end{array}$ & $\begin{array}{r}37.29 \\
-10.27 \\
-10.27\end{array}$ & $\begin{array}{l}37.29 \\
22.35 \\
37.29\end{array}$ & $\begin{array}{r}1 \\
20 \\
21\end{array}$ \\
\hline Snow & $\begin{array}{c}1 \\
2 \\
1 \& 2\end{array}$ & $\begin{array}{l}12.24 \\
10.02 \\
10.60\end{array}$ & $\begin{array}{l}15.25 \\
12.05 \\
12.96\end{array}$ & $\begin{array}{l}14.91 \\
17.87 \\
17.08\end{array}$ & $\begin{array}{r}0.56 \\
-22.99 \\
-22.99\end{array}$ & $\begin{array}{l}65.58 \\
88.12 \\
88.12\end{array}$ & $\begin{array}{r}31 \\
78 \\
109\end{array}$ \\
\hline & e. $F$ & ipitat & Rate & $\mathrm{n} / \mathrm{hr} \mathbf{r}$ & & & \\
\hline $0.0-1.0$ & $\begin{array}{c}1 \\
2 \\
1 \& 2\end{array}$ & $\begin{array}{l}18.06 \\
22.76 \\
21.08\end{array}$ & $\begin{array}{l}18.50 \\
22.92 \\
21.36\end{array}$ & $\begin{array}{l}23.46 \\
30.12 \\
28.00\end{array}$ & $\begin{array}{r}-119.79 \\
-27.45 \\
-119.79\end{array}$ & $\begin{array}{l}138.03 \\
333.25 \\
333.25\end{array}$ & $\begin{array}{l}158 \\
289 \\
447\end{array}$ \\
\hline $1.1-2.5$ & $\begin{array}{c}1 \\
2 \\
1 \& 2\end{array}$ & $\begin{array}{l}14.35 \\
10.93 \\
12.24\end{array}$ & $\begin{array}{l}15.64 \\
11.58 \\
13.18\end{array}$ & $\begin{array}{l}24.99 \\
16.63 \\
20.39\end{array}$ & $\begin{array}{l}-82.86 \\
-19.08 \\
-82.86\end{array}$ & $\begin{array}{r}186.58 \\
88.12 \\
186.58\end{array}$ & $\begin{array}{l}113 \\
174 \\
287\end{array}$ \\
\hline $2.6-6.0$ & $\begin{array}{c}1 \\
2 \\
1 \& 2\end{array}$ & $\begin{array}{l}7.10 \\
4.30 \\
5.22\end{array}$ & $\begin{array}{l}7.47 \\
4.83 \\
5.72\end{array}$ & $\begin{array}{r}8.56 \\
10.76 \\
10.12\end{array}$ & $\begin{array}{r}-5.44 \\
-22.99 \\
-22.99\end{array}$ & $\begin{array}{l}34.90 \\
45.30 \\
45.30\end{array}$ & $\begin{array}{r}56 \\
110 \\
166\end{array}$ \\
\hline $6.1-12.0$ & $\begin{array}{c}1 \\
2 \\
1 \& 2\end{array}$ & $\begin{array}{r}17.80 \\
1.57 \\
11.08\end{array}$ & $\begin{array}{r}10.13 \\
1.89 \\
5.09\end{array}$ & $\begin{array}{r}18.11 \\
6.51 \\
12.90\end{array}$ & $\begin{array}{r}-7.28 \\
-10.18 \\
-10.18\end{array}$ & $\begin{array}{l}83.64 \\
25.73 \\
83.64\end{array}$ & $\begin{array}{l}26 \\
41 \\
67\end{array}$ \\
\hline$>12.0$ & $\begin{array}{c}1 \\
2 \\
1 \& 2\end{array}$ & $\begin{array}{r}7.41 \\
-2.14 \\
2.09\end{array}$ & $\begin{array}{r}7.35 \\
-1.82 \\
2.76\end{array}$ & $\begin{array}{l}7.16 \\
5.19 \\
7.69\end{array}$ & $\begin{array}{r}-3.04 \\
-11.13 \\
-11.13\end{array}$ & $\begin{array}{r}17.33 \\
4.70 \\
17.33\end{array}$ & $\begin{array}{r}9 \\
9 \\
18\end{array}$ \\
\hline
\end{tabular}


TABLE 4 (continued) Excess Conductivity

Wt.

\begin{tabular}{|c|c|c|c|c|c|c|c|}
\hline Variable & Year & Mean & Mean & S.D. & Min. & Max. & $\mathrm{n}$. \\
\hline & f. & Speed & $\sec ^{-1}$ & t 108 & & & \\
\hline \multirow[t]{3}{*}{$0-2$} & 1 & 5.46 & 11.47 & 9.93 & -4.97 & 32.74 & 18 \\
\hline & 2 & -1.23 & 13.06 & 19.34 & -22.99 & 54.42 & 19 \\
\hline & $1 \& 2$ & 2.93 & 12.25 & 15.31 & -22.99 & 54.42 & 37 \\
\hline \multirow[t]{3}{*}{$3-5$} & 1 & 15.88 & 13.31 & 16.66 & -82.86 & 79.39 & 134 \\
\hline & 2 & 6.61 & 13.76 & 16.34 & -19.08 & 80.88 & 163 \\
\hline & $1 \& 2$ & 13.09 & 13.56 & 16.46 & -82.86 & 80.88 & 297 \\
\hline \multirow[t]{3}{*}{$6-9$} & 1 & 13.04 & 15.62 & 27.62 & -119.79 & $186: 58$ & 131 \\
\hline & 2 & 6.76 & $15: 51$ & 29.73 & $-1 \kappa .16$ & 333.75 & 282 \\
\hline & $1 \dot{\&} 2$ & 8.34. & 15.34 & 29.04 & $-1 \perp y^{\prime} . / y$ & 333.23 & 413 \\
\hline \multirow{3}{*}{$\geq 10$} & 1 & 11.79 & 17.76 & 21.55 & -5.44 & 119.08 & .79 \\
\hline & 2 & 8.31 & 16.51 & 21.17 & -27.45 & 104.57 & 140 \\
\hline & $1 \& 2$ & 9.74 & 16.96 & 21.27 & -27.45 & 119.08 & 219 \\
\hline
\end{tabular}

g. Wind Direction at $108 \mathrm{~m}$

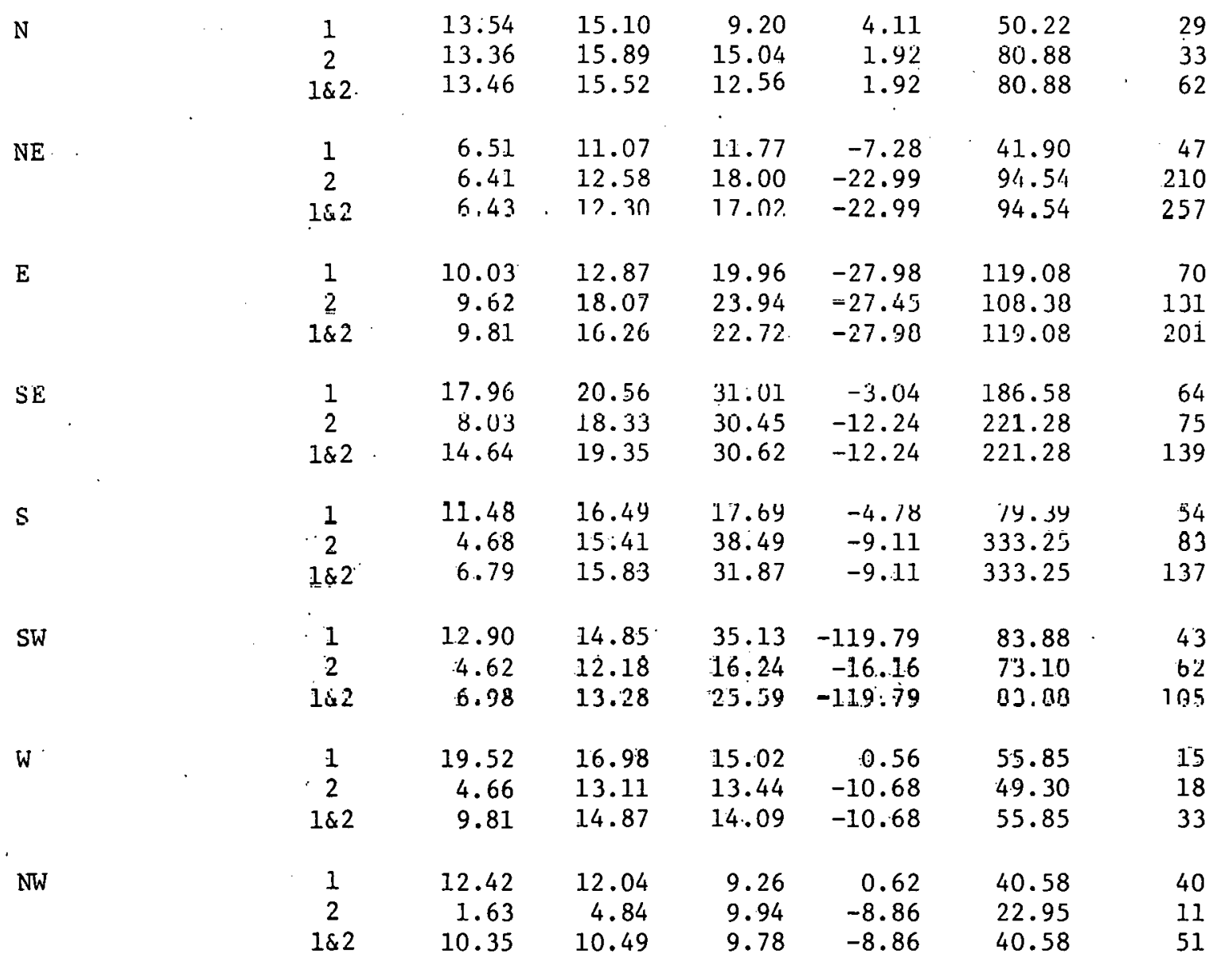


TABLE 4 (continued) Excess Conductivity

\begin{tabular}{|c|c|c|c|c|c|c|c|}
\hline Variable & Year & $\begin{array}{r}\text { Wt. } \\
\text { Mean }\end{array}$ & Mean & S.D. & Min. & Max. & n. \\
\hline & \multicolumn{7}{|c|}{ Temperature $\left({ }^{\circ} \mathrm{C}\right)$} \\
\hline-10.0 to -5.1 & $\begin{array}{c}1 \\
2 \\
1 \& 2\end{array}$ & $\begin{array}{l}33.69 \\
49.30 \\
33.78\end{array}$ & $\begin{array}{l}33.69 \\
49.30 \\
41.49\end{array}$ & $\begin{array}{c}- \\
- \\
11.04\end{array}$ & $\begin{array}{l}33.69 \\
49.30 \\
33.69\end{array}$ & $\begin{array}{l}33.69 \\
49.30 \\
49.30\end{array}$ & $\begin{array}{l}1 \\
1 \\
2\end{array}$ \\
\hline-5.0 to -0.1 & $\begin{array}{c}1 \\
2 \\
1 \& 2\end{array}$ & $\begin{array}{r}16.34 \\
9.03 \\
10.23\end{array}$ & $\begin{array}{r}18.85 \\
9.22 \\
11.24\end{array}$ & $\begin{array}{l}15.58 \\
11.16 \\
12.76\end{array}$ & $\begin{array}{r}3.90 \\
-10.27 \\
-10.27\end{array}$ & $\begin{array}{l}65.58 \\
52.73 \\
65.58\end{array}$ & $\begin{array}{r}21 \\
79 \\
100\end{array}$ \\
\hline 0.0 to 4.9 & $\begin{array}{c}1 \\
2 \\
1 \& 2\end{array}$ & $\begin{array}{r}10.31 \\
4.68 \\
6.89\end{array}$ & $\begin{array}{l}14.32 \\
10.61 \\
11.94\end{array}$ & $\begin{array}{l}12.63 \\
17.59 \\
16.04\end{array}$ & $\begin{array}{r}-0.90 \\
-22.99 \\
-22.99\end{array}$ & $\begin{array}{l}57.86 \\
88.12 \\
88.12\end{array}$ & $\begin{array}{r}56 \\
100 \\
156\end{array}$ \\
\hline 5.0 to 9.9 & $\begin{array}{c}1 \\
2 \\
1 \& 2\end{array}$ & $\begin{array}{l}5.10 \\
7.51 \\
6.53\end{array}$ & $\begin{array}{l}11.32 \\
21.82 \\
17.28\end{array}$ & $\begin{array}{l}21.45 \\
41.93 \\
34.92\end{array}$ & $\begin{array}{r}-7.28 \\
-27.45 \\
-27.45\end{array}$ & $\begin{array}{l}186.58 \\
333.25 \\
333.25\end{array}$ & $\begin{array}{r}96 \\
126 \\
222\end{array}$ \\
\hline 10.0 to 14.9 & $\begin{array}{c}1 \\
2 \\
1 \& 2\end{array}$ & $\begin{array}{r}12.41 \\
9.00 \\
9.91\end{array}$ & $\begin{array}{l}17.94 \\
14.84 \\
15.77\end{array}$ & $\begin{array}{l}20.51 \\
17.87 \\
18.71\end{array}$ & $\begin{array}{r}-4.78 \\
-10.18 \\
-10.18\end{array}$ & $\begin{array}{l}138.03 \\
108.38 \\
138.03\end{array}$ & $\begin{array}{r}75 \\
176 \\
251\end{array}$ \\
\hline 15.0 to 19.9 & $\begin{array}{c}1 \\
2 \\
1 \& 2\end{array}$ & $\begin{array}{r}13.29 \\
5.63 \\
8.02\end{array}$ & $\begin{array}{l}20.48 \\
14.49 \\
16.36\end{array}$ & $\begin{array}{l}17.71 \\
16.36 \\
16.94\end{array}$ & $\begin{array}{r}1.15 \\
-16.16 \\
-16.16\end{array}$ & $\begin{array}{l}83.88 \\
59.24 \\
83.88\end{array}$ & $\begin{array}{r}34 \\
75 \\
109\end{array}$ \\
\hline 20.0 to 24.9 & $\begin{array}{c}1 \\
2 \\
1 \& 2\end{array}$ & $\begin{array}{r}18.41 \\
3.06 \\
13.85\end{array}$ & $\begin{array}{l}13.10 \\
13.96 \\
13.49\end{array}$ & $\begin{array}{l}30.53 \\
16.44 \\
25.16\end{array}$ & $\begin{array}{r}-119.79 \\
-11.13 \\
-119.79\end{array}$ & $\begin{array}{r}119.08 \\
73.10 \\
119.08\end{array}$ & $\begin{array}{r}77 \\
62 \\
139\end{array}$ \\
\hline 25.0 to 29.9 & $\begin{array}{c}1 \\
2 \\
1 \& 2\end{array}$ & $\begin{array}{r}19.30 \\
8.15 \\
114.36\end{array}$ & $\begin{array}{l}35.40 \\
19.25 \\
24.63\end{array}$ & $\begin{array}{l}28.92 \\
15.13 \\
19.34\end{array}$ & $\begin{array}{r}14.95 \\
0.31 \\
0.31\end{array}$ & $\begin{array}{l}55.85 \\
33.36 \\
55.85\end{array}$ & $\begin{array}{l}2 \\
4 \\
6\end{array}$ \\
\hline
\end{tabular}


TABLE 5 Variation in $\mathrm{pH}$

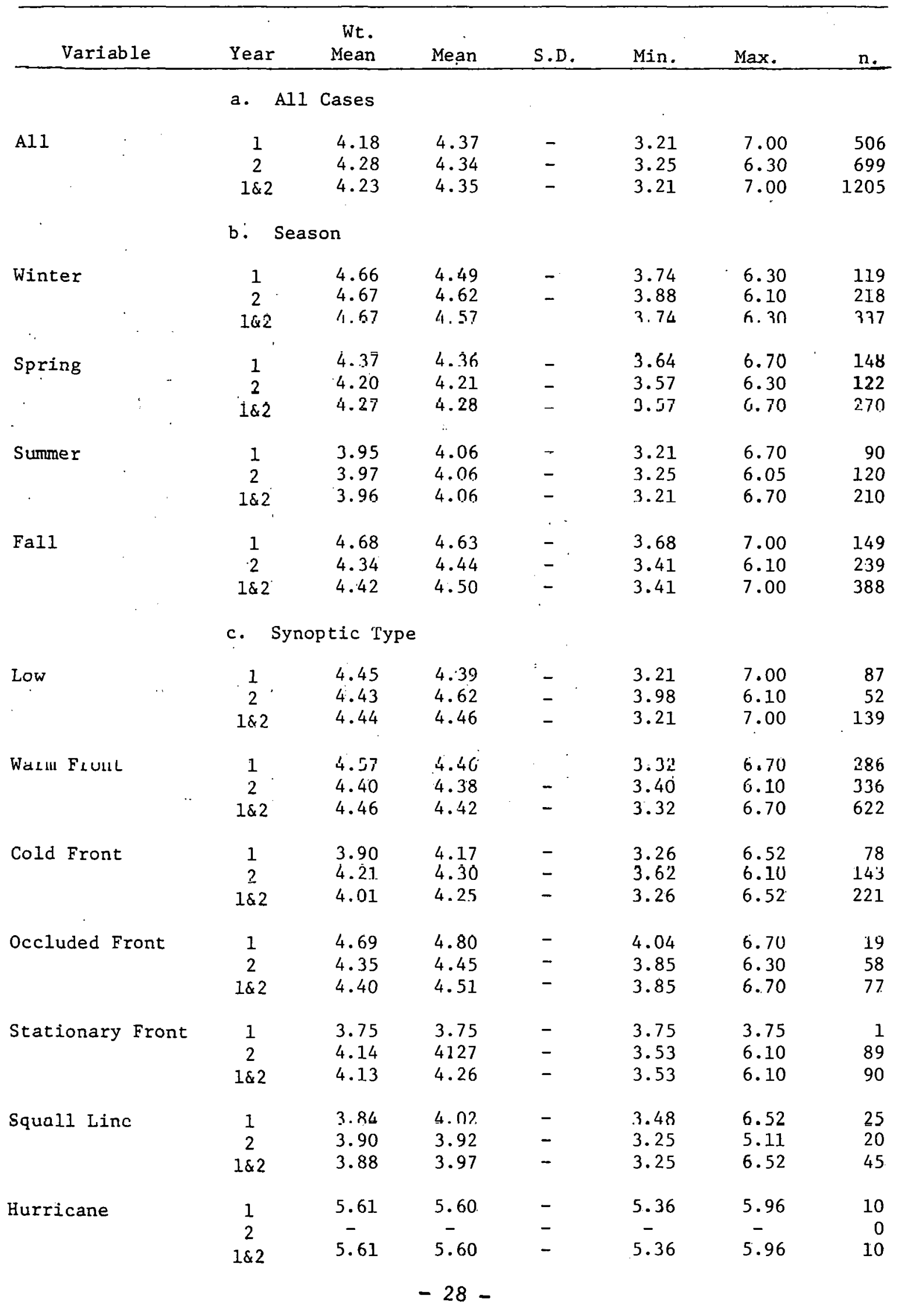


TABLE 5 (continued) pH

\begin{tabular}{|c|c|c|c|c|c|c|c|}
\hline Variable & Year & $\begin{array}{l}\text { Wt. } \\
\text { Mean }\end{array}$ & Mean & S.D. & Min. & Max. & $\mathrm{n}$. \\
\hline & \multicolumn{3}{|c|}{ Precipitation Type } & & & & \\
\hline Rain & $\begin{array}{c}1 \\
2 \\
1 \& 2\end{array}$ & $\begin{array}{l}4.53 \\
4.41 \\
4.45\end{array}$ & $\begin{array}{l}4.50 \\
4.44 \\
4.46\end{array}$ & $\begin{array}{l}- \\
- \\
-\end{array}$ & $\begin{array}{l}3.32 \\
3.55 \\
3.32\end{array}$ & $\begin{array}{l}7.00 \\
6.10 \\
7.00\end{array}$ & $\begin{array}{r}346 \\
458 \\
804\end{array}$ \\
\hline Rainshower & $\begin{array}{c}1 \\
2 \\
1 \& 2\end{array}$ & $\begin{array}{l}4.28 \\
3.92 \\
4.04\end{array}$ & $\begin{array}{l}4.17 \\
3.99 \\
4.06\end{array}$ & $\begin{array}{l}- \\
-\end{array}$ & $\begin{array}{l}3.56 \\
3.25 \\
3.25\end{array}$ & $\begin{array}{l}6.70 \\
6.30 \\
6.70\end{array}$ & $\begin{array}{r}59 \\
80 \\
139\end{array}$ \\
\hline Thundershower & $\begin{array}{c}1 \\
2 \\
1 \& 2\end{array}$ & $\begin{array}{l}3.92 \\
4.00 \\
3.95\end{array}$ & $\begin{array}{l}3.89 \\
4.04 \\
3.95\end{array}$ & $\begin{array}{l}- \\
-\end{array}$ & $\begin{array}{l}3.21 \\
3.63 \\
3.21\end{array}$ & $\begin{array}{l}5.96 \\
4.88 \\
5.96\end{array}$ & $\begin{array}{l}35 \\
31 \\
66\end{array}$ \\
\hline Mixed & $\begin{array}{c}1 \\
2 \\
1 \& 2\end{array}$ & $\begin{array}{l}4.18 \\
4.61 \\
4.53\end{array}$ & $\begin{array}{l}4.26 \\
4.53 \\
4.46\end{array}$ & $\begin{array}{l}- \\
-\end{array}$ & $\begin{array}{l}4.04 \\
3.90 \\
3.90\end{array}$ & $\begin{array}{l}5.16 \\
5.92 \\
5.92\end{array}$ & $\begin{array}{r}5 \\
21 \\
26\end{array}$ \\
\hline Sleet & $\begin{array}{c}1 \\
2 \\
1 \& 2\end{array}$ & $\begin{array}{l}4.69 \\
4.47 \\
4.48\end{array}$ & $\begin{array}{l}4.69 \\
4.46 \\
4.47\end{array}$ & $\begin{array}{l}- \\
-\end{array}$ & $\begin{array}{l}4.69 \\
4.11 \\
4.11\end{array}$ & $\begin{array}{l}4.69 \\
5.54 \\
5.54\end{array}$ & $\begin{array}{r}1 \\
20 \\
21\end{array}$ \\
\hline Snow & $\begin{array}{c}1 \\
2 \\
1 \& 2\end{array}$ & $\begin{array}{l}4.52 \\
4.52 \\
4.52\end{array}$ & $\begin{array}{l}4.50 \\
4.49 \\
4.49\end{array}$ & $\begin{array}{l}- \\
- \\
-\end{array}$ & $\begin{array}{l}3.82 \\
3.85 \\
3.82\end{array}$ & $\begin{array}{l}6.10 \\
5.64 \\
6.10\end{array}$ & $\begin{array}{r}60 \\
89 \\
149\end{array}$ \\
\hline & e. & pitat & Rate & $/ \mathrm{hr}$ ) & & & \\
\hline $0.0-1.0$ & $\begin{array}{c}1 \\
2 \\
1 \& 2\end{array}$ & $\begin{array}{l}4.32 \\
4.34 \\
4.33\end{array}$ & $\begin{array}{l}4.36 \\
4.37 \\
4.37\end{array}$ & $\begin{array}{l}- \\
-\end{array}$ & $\begin{array}{l}3.26 \\
3.25 \\
3.25\end{array}$ & $\begin{array}{l}7.00 \\
6.30 \\
7.00\end{array}$ & $\begin{array}{l}291 \\
361 \\
652\end{array}$ \\
\hline $1.1-2.5$ & $\begin{array}{c}1 \\
2 \\
1 \& 2\end{array}$ & $\begin{array}{l}4.38 \\
4.30 \\
4.33\end{array}$ & $\begin{array}{l}4.36 \\
4.30 \\
4.32\end{array}$ & $\begin{array}{l}- \\
-\end{array}$ & $\begin{array}{l}3.21 \\
3.41 \\
3.21\end{array}$ & $\begin{array}{l}6.40 \\
5.64 \\
6.40\end{array}$ & $\begin{array}{l}122 \\
176 \\
298\end{array}$ \\
\hline $2.6=6.0$ & $\begin{array}{c}1 \\
2 \\
1 \& 2\end{array}$ & $\begin{array}{l}4.52 \\
4.33 \\
4.38\end{array}$ & $\begin{array}{l}4.51 \\
4.34 \\
4.39\end{array}$ & $\begin{array}{l}- \\
- \\
-\end{array}$ & $\begin{array}{l}3.66 \\
3.69 \\
3.66\end{array}$ & $\begin{array}{l}6.70 \\
5.46 \\
6.70\end{array}$ & $\begin{array}{r}57 \\
112 \\
169\end{array}$ \\
\hline $6.1-12.0$ & $\begin{array}{c}1 \\
2 \\
1 \& 2\end{array}$ & $\begin{array}{l}4.00 \\
4.38 \\
4.12\end{array}$ & $\begin{array}{l}4.34 \\
4.40 \\
4.38\end{array}$ & $\begin{array}{l}- \\
-\end{array}$ & $\begin{array}{l}3.48 \\
3.77 \\
3.48\end{array}$ & $\begin{array}{l}5.96 \\
5.28 \\
5.96\end{array}$ & $\begin{array}{l}26 \\
41 \\
67\end{array}$ \\
\hline$>12.0$ & $\begin{array}{c}1 \\
2 \\
1 \& 2\end{array}$ & $\begin{array}{l}4.17 \\
3.98 \\
4.05\end{array}$ & $\begin{array}{l}4.16 \\
4.01 \\
4.08\end{array}$ & $\begin{array}{l}- \\
-\end{array}$ & $\begin{array}{l}1.72 \\
3.63 \\
3.63\end{array}$ & $\begin{array}{l}5.44^{\circ} \\
5.12 \\
5.46\end{array}$ & $\begin{array}{r}10 \\
9 \\
19\end{array}$ \\
\hline
\end{tabular}


TABLE 5 (continued) $\mathrm{pH}$

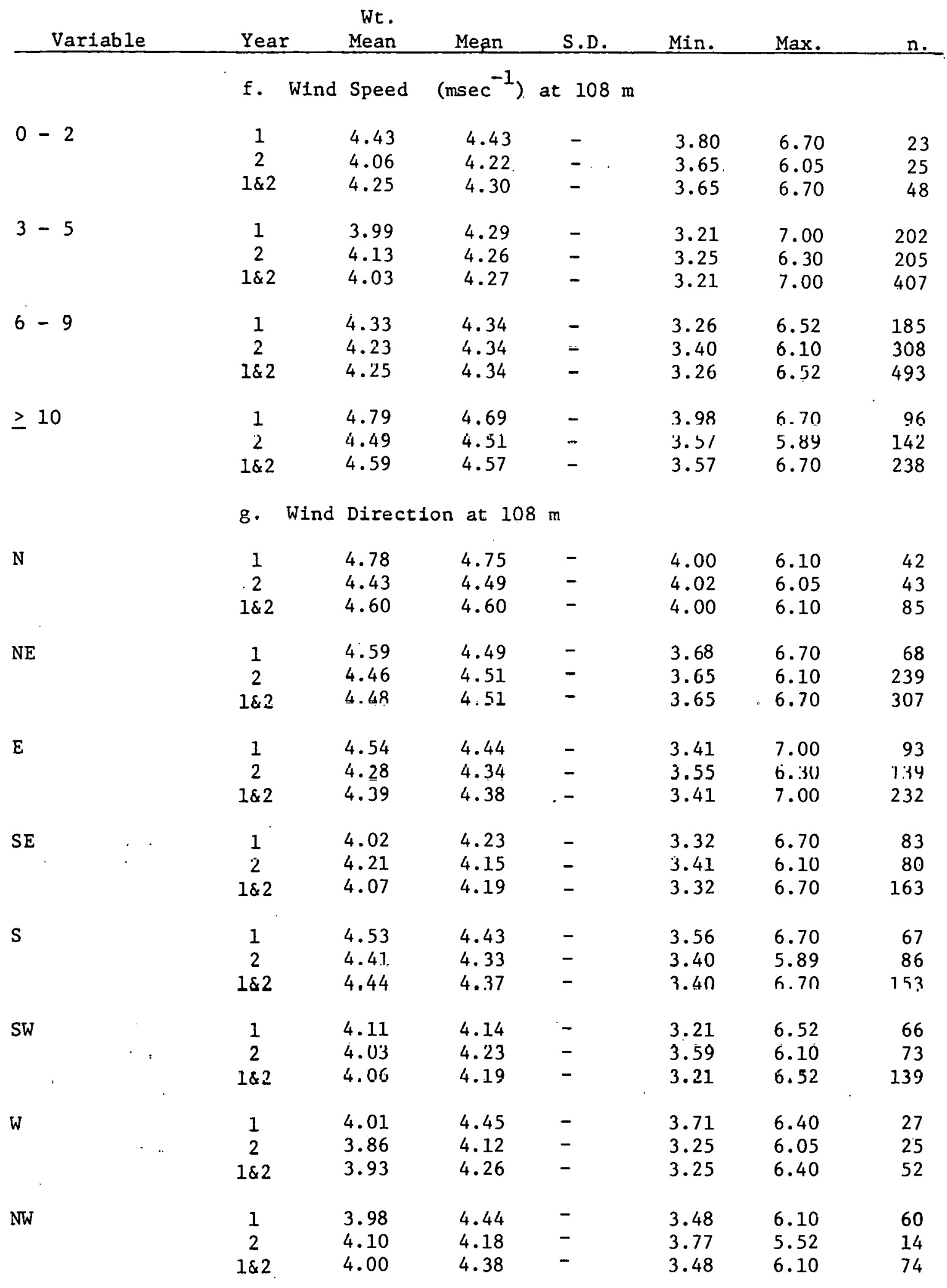


TABLE 5 (continued) $\mathrm{pH}$

\begin{tabular}{|c|c|c|c|c|c|c|c|}
\hline Variable & Year & $\begin{array}{l}\text { Wt. } \\
\text { Mean }\end{array}$ & Mean & S.D. & Min. & Max. & n. \\
\hline \multicolumn{8}{|c|}{ h. Temperature $\left({ }^{\circ} \mathrm{C}\right)$} \\
\hline \multirow{3}{*}{-10.0 to -5.1} & 1 & 4.25 & 4.30 & - & 3.98 & 4.97 & 6 \\
\hline & 2 & 4.07 & 4.07 & - & 4.07 & 4.07 & 1 \\
\hline & $1 \& 2$ & 4.25 & 4.26 & - & 3.98 & 4.97 & 7 \\
\hline \multirow[t]{3}{*}{-5.0 to -0.1} & 1 & 4.50 & 4.51 & - & 3.82 & 6.10 & 44 \\
\hline & 2 & 4.66 & 4.57 & - & 3.88 & 5.64 & 91 \\
\hline & $1 \& 2$ & 4.62 & 4.55 & - & 3.82 & 6.10 & 135 \\
\hline \multirow[t]{3}{*}{0.0 to 4.9} & 1 & 4.53 & 4.45 & - & 3.74 & 5.92 & 80 \\
\hline & 2 & 4.43 & 4.53 & - & 3.85 & 6.10 & 115 \\
\hline & $1 \& 2$ & 4.47 & 4.50 & - & 3.74 & 6.10 & 195 \\
\hline \multirow[t]{3}{*}{5.0 to 9.9} & 1 & 4.52 & 4.48 & - & 3.80 & 6.70 & 120 \\
\hline & 2 & 4.38 & 4.39 & - & 3.57 & 6.10 & 141 \\
\hline & $1 \& 2$ & .4 .43 & 4.43 & - & 3.57 & 6.70 & 261 \\
\hline \multirow[t]{3}{*}{10.0 to 14.9} & 1 & 4.45 & 4.44 & - & 3.64 & 6.70 & 94 \\
\hline & 2 & 4.45 & 4.43 & - & 3.55 & 6.30 & 191 \\
\hline & $1 \& 2$ & 4.45 & 4.43 & - & 3.55 & 6.70 & 285 \\
\hline \multirow[t]{3}{*}{15.0 to 19.9} & 1 & 4.36 & 4.38 & - & 3.66 & 6.40 & 53 \\
\hline & 2 & 4.13 & 4.13 & - & 3.53 & 6.10 & 84 \\
\hline & $1 \& 2$ & 4.20 & 4.21 & - & 3.53 & 6.40 & 137 \\
\hline \multirow[t]{3}{*}{20.0 to 24.9} & 1 & 3.98 & 4.17 & - & 3.21 & 7.00 & 107 \\
\hline & 2 & 3.94 & 4.08 & - & 3.40 & 6.05 & 71 \\
\hline & $1 \& 2$ & 3.97 & 4.13 & - & 3.21 & 7.00 & 178 \\
\hline \multirow[t]{3}{*}{25.0 to 29.9} & 1 & 3.94 & 3.82 & - & 3.71 & 3.98 & 2 \\
\hline & 2 & 3.89 & 3.69 & - & 3.25 & 4.52 & 5 \\
\hline & $1 \& 2$ & 3.92 & 3.73 & - & 3.25 & 4.52 & 7 \\
\hline
\end{tabular}




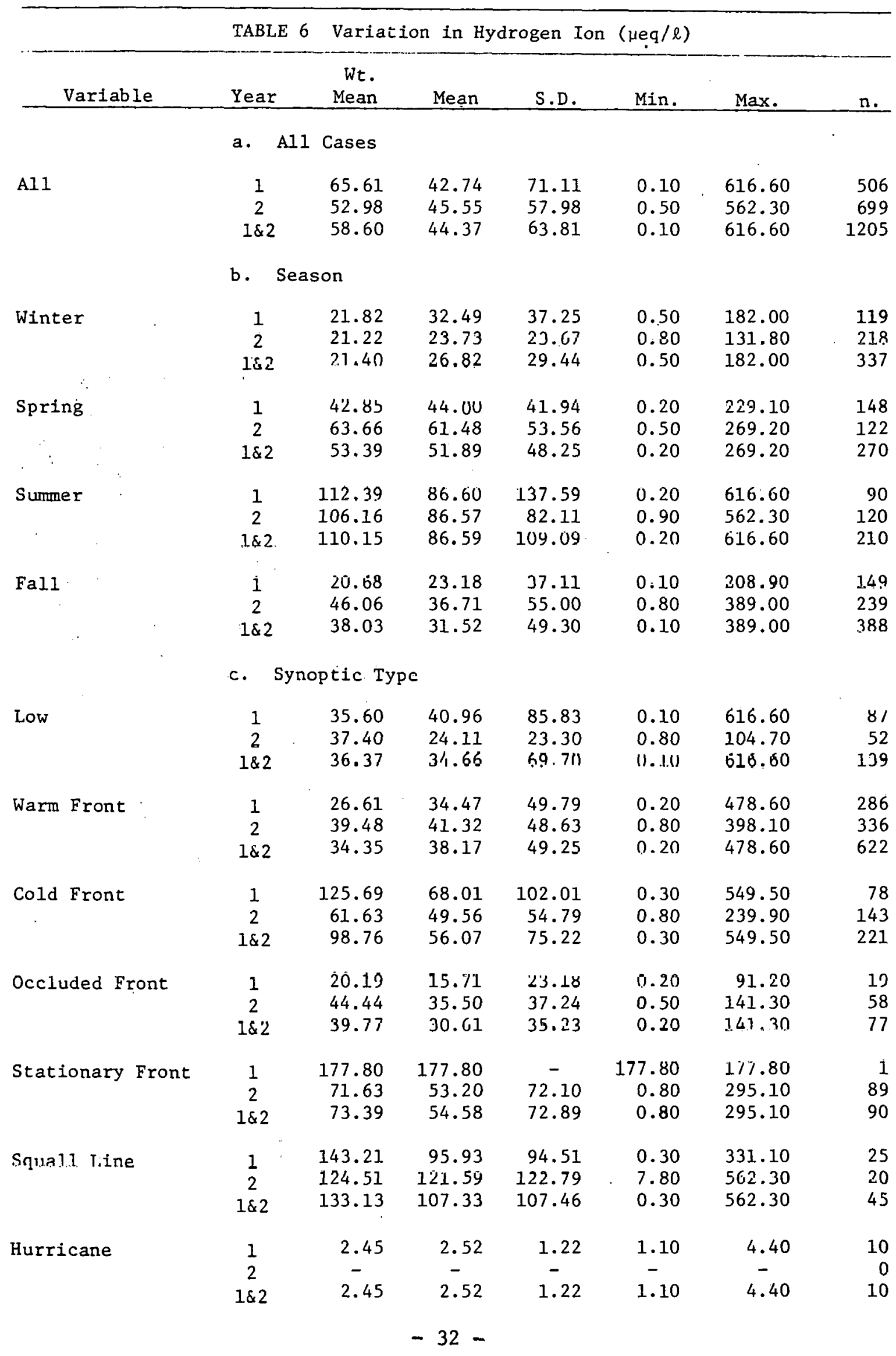


TABLE 6 (continued) $\mathrm{H}$ Ion

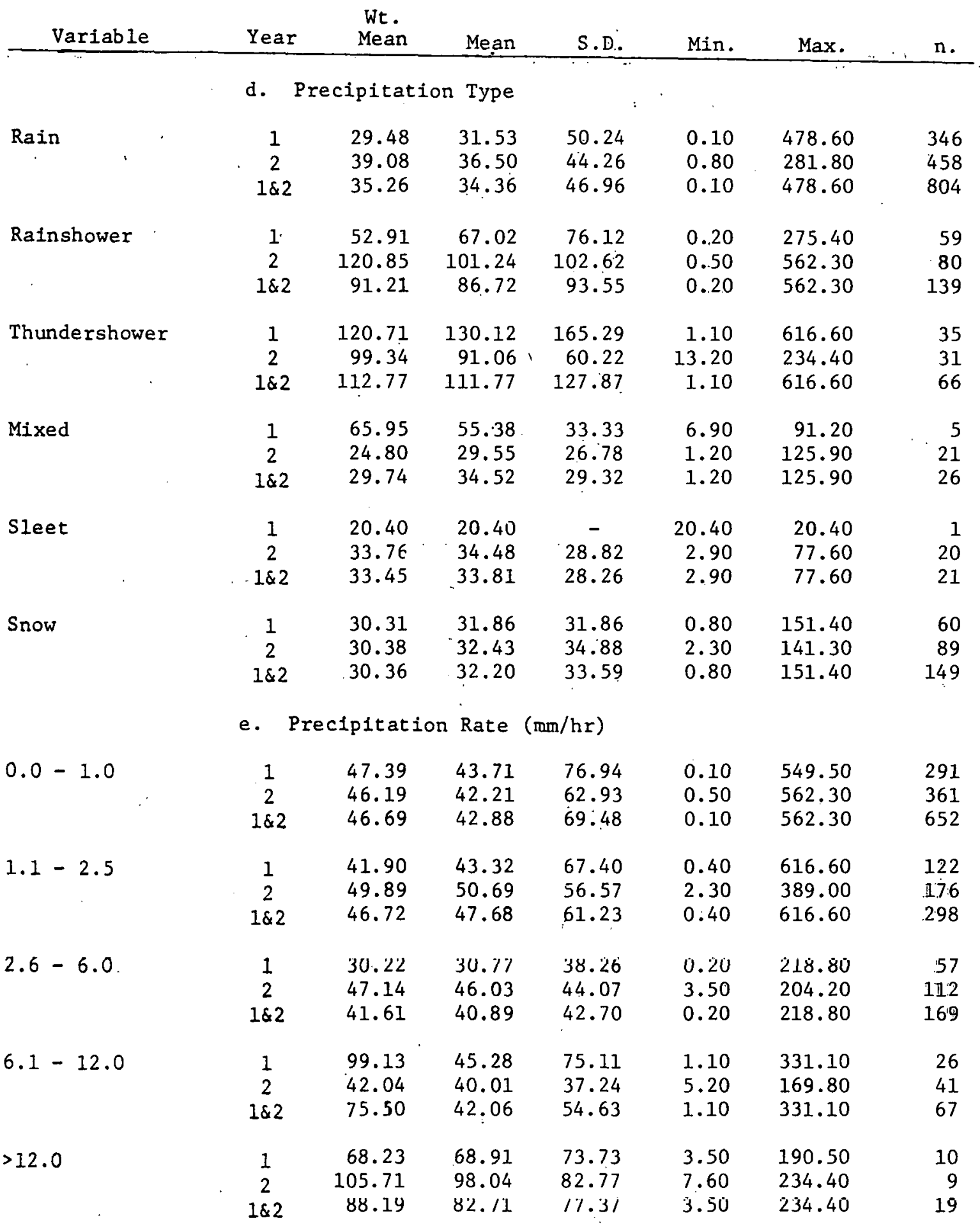


TABLE 6 (continued) H Ion

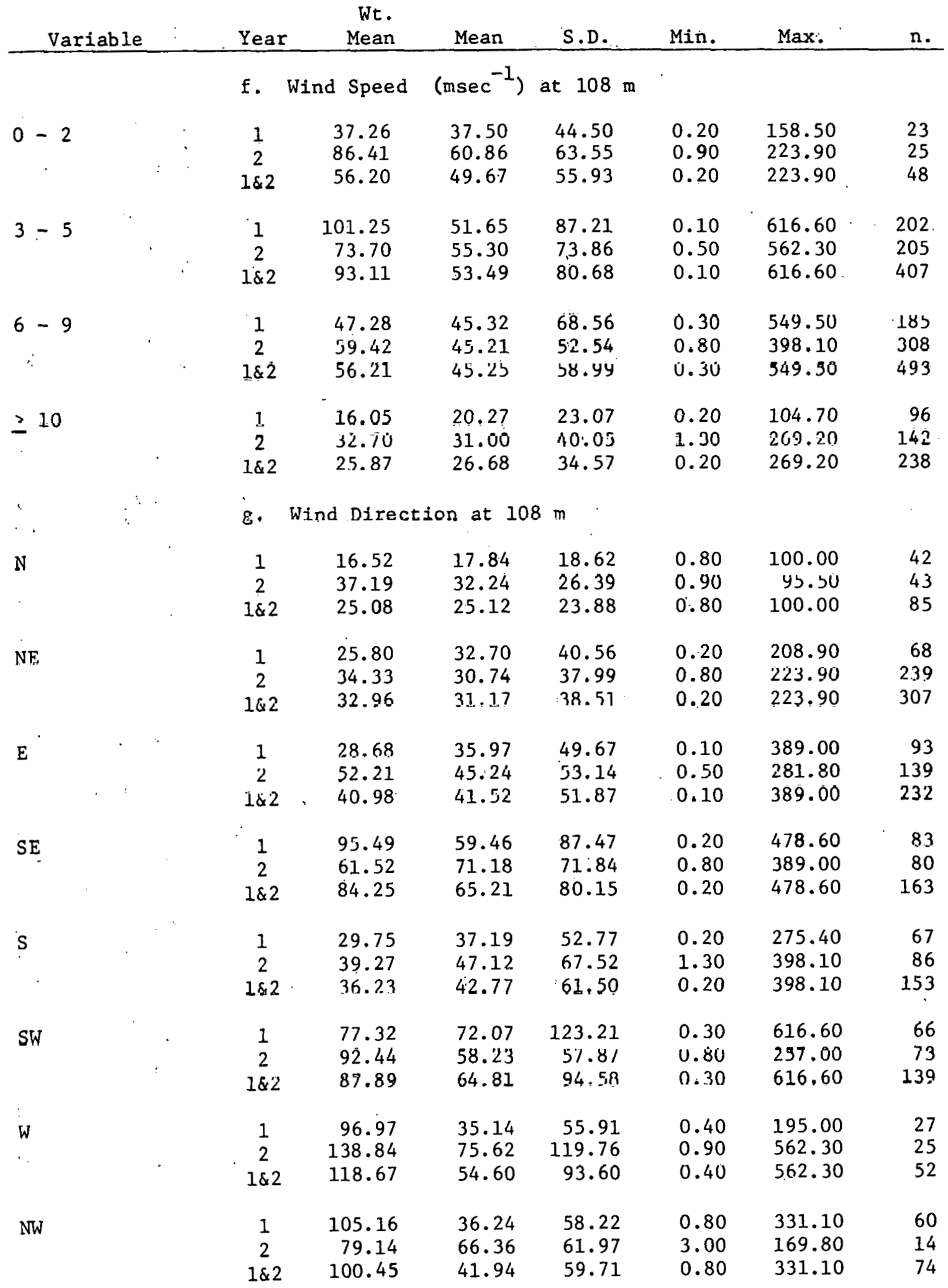


TABLE 6 (continued) H Ion

\begin{tabular}{|c|c|c|c|c|c|c|c|}
\hline Variable & Year & $\begin{array}{l}\text { Wt. } \\
\text { Mean }\end{array}$ & Mean & S.D. & Min. & Max. & $\mathrm{n}$. \\
\hline & h. I & nperature & $\left({ }^{\circ} \mathrm{C}\right)$ & & & & \\
\hline-10.0 to -5.1 & $\begin{array}{c}1 \\
2 \\
1 \& 2\end{array}$ & $\begin{array}{l}56.58 \\
85.10 \\
56.61\end{array}$ & $\begin{array}{l}49.85 \\
85.10 \\
54.89\end{array}$ & $\begin{array}{c}38.62 \\
- \\
37.69\end{array}$ & $\begin{array}{l}10.70 \\
85.10 \\
10.70\end{array}$ & $\begin{array}{r}104.70 \\
85.10 \\
104.70\end{array}$ & $\begin{array}{l}6 \\
1 \\
7\end{array}$ \\
\hline-5.0 to -0.1 & $\begin{array}{c}1 \\
2 \\
1 \& 2\end{array}$ & $\begin{array}{l}31.87 \\
21.92 \\
24.23\end{array}$ & $\begin{array}{l}30.89 \\
26.66 \\
28.04\end{array}$ & $\begin{array}{l}28.49 \\
28.75 \\
28.63\end{array}$ & $\begin{array}{l}0.80 \\
2.30 \\
0.80\end{array}$ & $\begin{array}{l}151.40 \\
131.80 \\
151.40\end{array}$ & $\begin{array}{r}44 \\
91 \\
135\end{array}$ \\
\hline 0.0 to 4.9 & $\begin{array}{c}1 \\
2 \\
1 \& 2\end{array}$ & $\begin{array}{l}29.36 \\
37.16 \\
33.94\end{array}$ & $\begin{array}{l}35.15 \\
29.70 \\
31.94\end{array}$ & $\begin{array}{l}37.91 \\
29.03 \\
32.98\end{array}$ & $\begin{array}{l}1.20 \\
0.80 \\
0.80\end{array}$ & $\begin{array}{l}182.00 \\
141.30 \\
182.00\end{array}$ & $\begin{array}{r}80 \\
115 \\
195\end{array}$ \\
\hline 5.0 to 9.9 & $\begin{array}{c}1 \\
2 \\
1 \& 2\end{array}$ & $\begin{array}{l}30.25 \\
41.68 \\
36.96\end{array}$ & $\begin{array}{l}33.42 \\
40.48 \\
37.23\end{array}$ & $\begin{array}{l}32.93 \\
48.55 \\
42.16\end{array}$ & $\begin{array}{l}0.20 \\
0.80 \\
0.20\end{array}$ & $\begin{array}{l}158.50 \\
269.20 \\
269.20\end{array}$ & $\begin{array}{l}120 \\
141 \\
261\end{array}$ \\
\hline 10.0 to 14.9 & $\begin{array}{c}1 \\
2 \\
1 \& 2\end{array}$ & $\begin{array}{l}35.56 \\
35.19 \\
35.29\end{array}$ & $\begin{array}{l}36.32 \\
37.32 \\
36.99\end{array}$ & $\begin{array}{l}48.09 \\
42.76 \\
44.51\end{array}$ & $\begin{array}{l}0.20 \\
0.50 \\
0.20\end{array}$ & $\begin{array}{l}229.10 \\
281.80 \\
281.80\end{array}$ & $\begin{array}{r}94 \\
191 \\
285\end{array}$ \\
\hline 15.0 to 19.9 & $\begin{array}{c}1 \\
2 \\
1 \& 2\end{array}$ & $\begin{array}{l}43.41 \\
73.40 \\
62.88\end{array}$ & $\begin{array}{l}41.97 \\
73.50 \\
61.30\end{array}$ & $\begin{array}{l}57.40 \\
74.23 \\
69.71\end{array}$ & $\begin{array}{l}0.40 \\
0.80 \\
0.40\end{array}$ & $\begin{array}{l}218.80 \\
295.10 \\
295.10\end{array}$ & $\begin{array}{r}53 \\
84 \\
137\end{array}$ \\
\hline 20.0 to 24.9 & $\begin{array}{c}1 \\
2 \\
1 \& 2\end{array}$ & $\begin{array}{l}104.00 \\
115.80 \\
107.48\end{array}$ & $\begin{array}{l}67.35 \\
82.88 \\
73.55\end{array}$ & $\begin{array}{r}129.05 \\
84.69 \\
113.44\end{array}$ & $\begin{array}{l}0.10 \\
0.90 \\
0.10\end{array}$ & $\begin{array}{l}616.60 \\
398.10 \\
616.60\end{array}$ & $\begin{array}{r}107 \\
71 \\
178\end{array}$ \\
\hline 25.0 to 29.9 & $\begin{array}{c}1 \\
2 \\
1 \& 2\end{array}$ & $\begin{array}{l}114.31 \\
127.75 \\
120.40\end{array}$ & $\begin{array}{l}149.85 \\
203.32 \\
188.04\end{array}$ & $\begin{array}{r}63.85 \\
206.42 \\
172.53\end{array}$ & $\begin{array}{r}104.70 \\
30.20 \\
30.20\end{array}$ & $\begin{array}{l}195.00 \\
562.30 \\
562.30\end{array}$ & $\begin{array}{l}2 \\
5 \\
7\end{array}$ \\
\hline
\end{tabular}




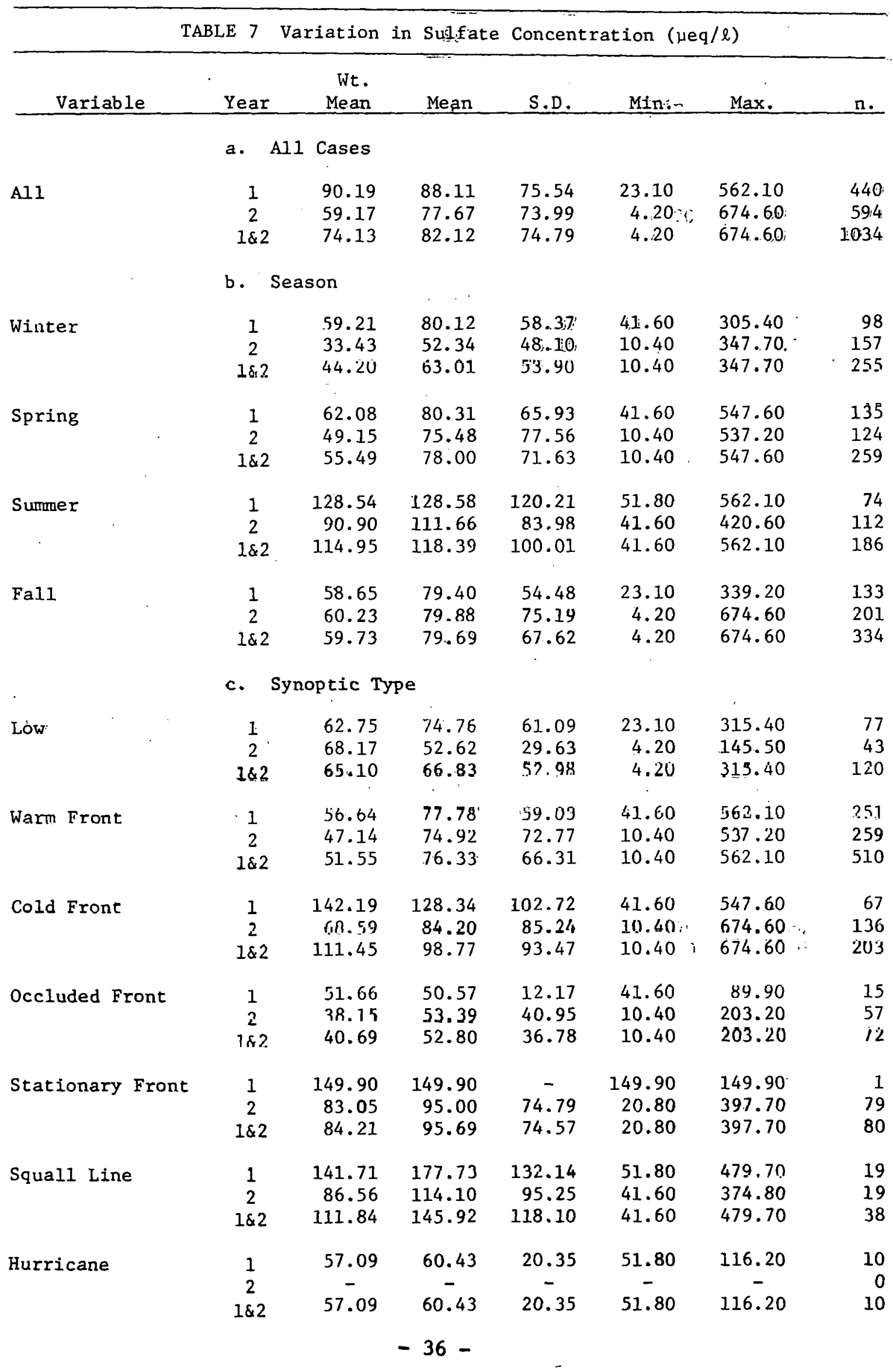


TABLE 7 (continued) Sulfate

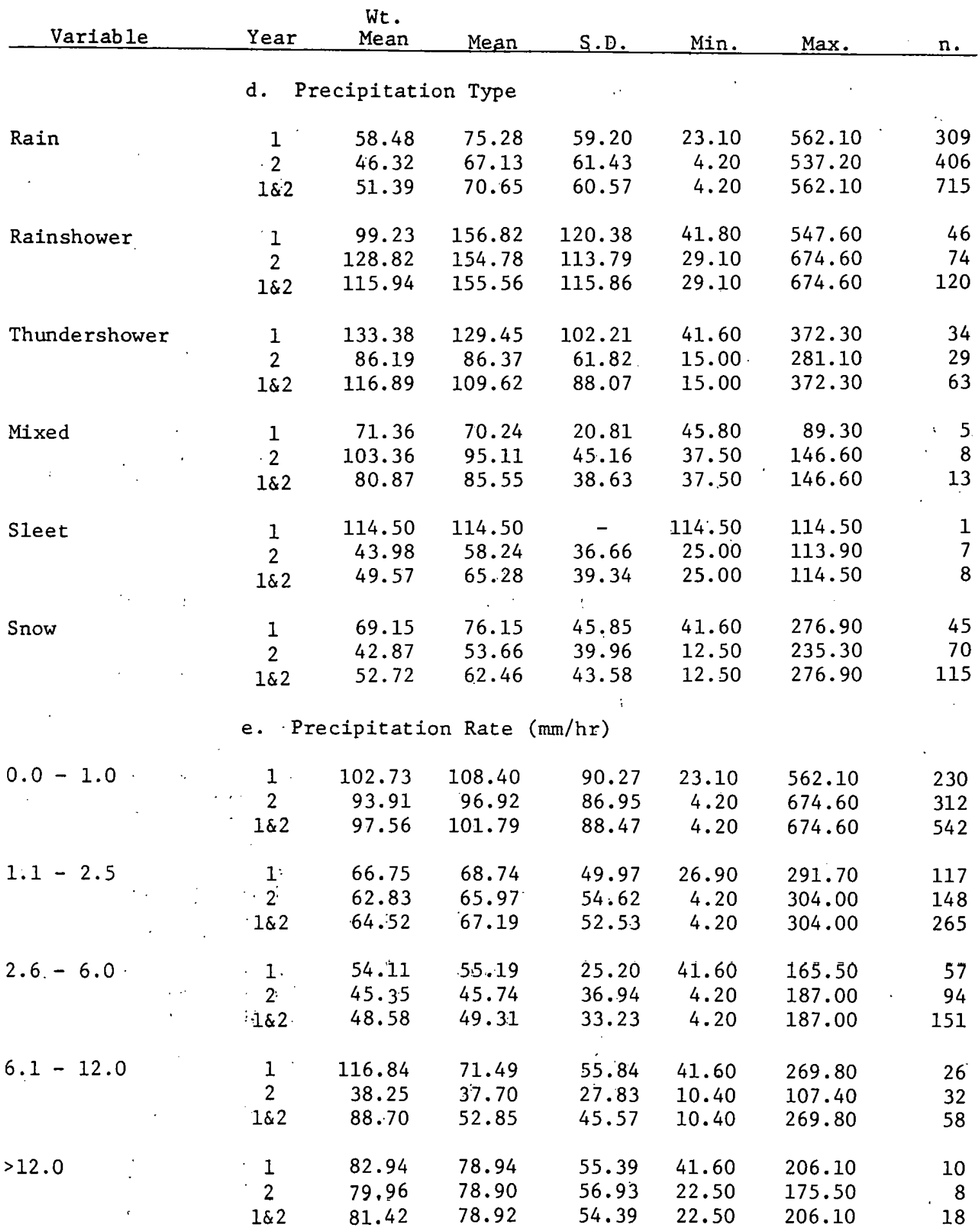


TABLE 7 (continued) Sulfate

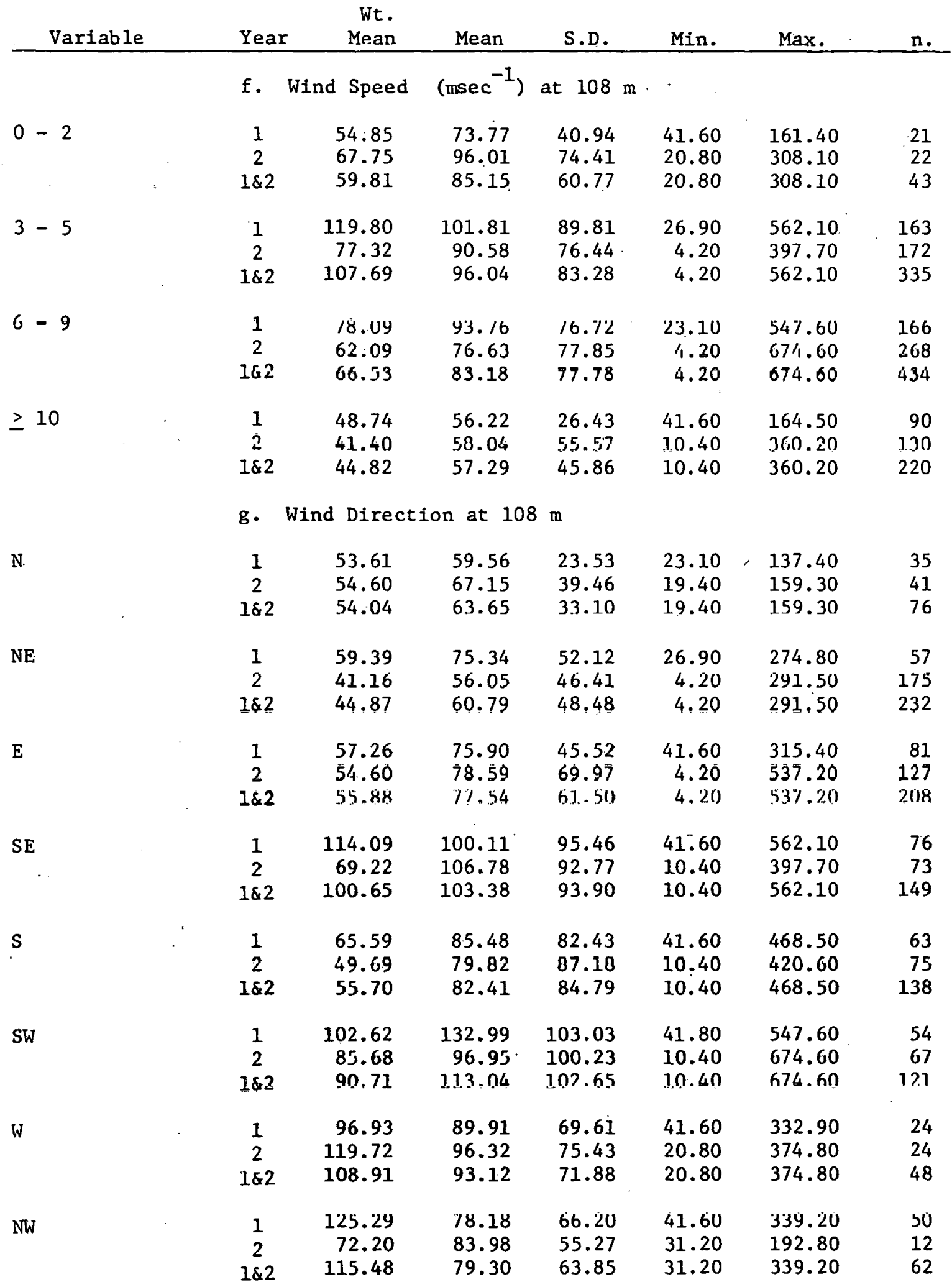




\begin{tabular}{|c|c|c|c|c|c|c|c|}
\hline Variable & Year & $\begin{array}{l}\text { Wt. } \\
\text { Mean }\end{array}$ & Mean & S.D. & Min. & Max. & n. \\
\hline & h. T & peratur & $\left({ }^{\circ} \mathrm{C}\right)$ & & & & \\
\hline \multirow[t]{2}{*}{-10.0 to -5.1} & $\begin{array}{l}1 \\
2\end{array}$ & $\begin{array}{l}128.41 \\
101.82\end{array}$ & $\begin{array}{l}123.90 \\
115.87\end{array}$ & $\begin{array}{l}57.42 \\
18.97\end{array}$ & $\begin{array}{l}83.30 \\
95.80\end{array}$ & $\begin{array}{l}164.50 \\
133.50\end{array}$ & $\begin{array}{l}2 \\
3\end{array}$ \\
\hline & $1 \& 2$ & 123.53 & 119.08 & 31.99 & 83.30 & 164.50 & 5 \\
\hline-5.0 to -0.1 & $\begin{array}{c}1 \\
2 \\
1 \& 2\end{array}$ & $\begin{array}{l}75.97 \\
40.53 \\
52.53\end{array}$ & $\begin{array}{l}80.71 \\
51.83 \\
62.66\end{array}$ & $\begin{array}{l}47.80 \\
40.73 \\
45.47\end{array}$ & $\begin{array}{l}41.60 \\
12.50 \\
12.50\end{array}$ & $\begin{array}{l}276.90 \\
235.30 \\
276.90\end{array}$ & $\begin{array}{l}33 \\
55 \\
88\end{array}$ \\
\hline 0.0 to 4.9 & $\begin{array}{c}1 \\
2 \\
1 \& 2\end{array}$ & $\begin{array}{l}60.13 \\
45.81 \\
53.21\end{array}$ & $\begin{array}{l}75.22 \\
64.22 \\
68.90\end{array}$ & $\begin{array}{l}49.45 \\
66.13 \\
59.69\end{array}$ & $\begin{array}{l}41.60 \\
10.40 \\
10.40\end{array}$ & $\begin{array}{l}305.40 \\
537.20 \\
537.20\end{array}$ & $\begin{array}{r}68 \\
92 \\
160\end{array}$ \\
\hline 5.0 to 9.9 & $\begin{array}{c}1 \\
2 \\
1 \& 2\end{array}$ & $\begin{array}{l}53.65 \\
39.92 \\
45.69\end{array}$ & $\begin{array}{l}73.35 \\
64.18 \\
68.44\end{array}$ & $\begin{array}{l}53.01 \\
60.09 \\
56.98\end{array}$ & $\begin{array}{l}41.60 \\
10.40 \\
10.40\end{array}$ & $\begin{array}{l}295.60 \\
360.20 \\
360.20\end{array}$ & $\begin{array}{l}110 \\
127 \\
237\end{array}$ \\
\hline 10.0 to 14.9 & $\begin{array}{c}1 \\
2 \\
1 \& 2\end{array}$ & $\begin{array}{l}63.37 \\
54.27 \\
57.08\end{array}$ & $\begin{array}{l}80.34 \\
75.93 \\
77.39\end{array}$ & $\begin{array}{l}54.52 \\
75.27 \\
69.02\end{array}$ & $\begin{array}{l}23.10 \\
10.40 \\
10.40\end{array}$ & $\begin{array}{l}266.50 \\
674.60 \\
674.60\end{array}$ & $\begin{array}{r}86 \\
174 \\
260\end{array}$ \\
\hline 15.0 to 19.9 & $\begin{array}{c}1 \\
2 \\
1 \& 2\end{array}$ & $\begin{array}{l}70.00 \\
68.78 \\
69.21\end{array}$ & $\begin{array}{l}113.56 \\
104.59 \\
108.04\end{array}$ & $\begin{array}{r}107.35 \\
86.90 \\
94.97\end{array}$ & $\begin{array}{l}41.80 \\
20.80 \\
20.80\end{array}$ & $\begin{array}{l}547.60 \\
397.70 \\
547.60\end{array}$ & $\begin{array}{r}47 \\
75 \\
122\end{array}$ \\
\hline 20.0 to 24.9 & $\begin{array}{c}1 \\
2 \\
1 \& 2\end{array}$ & $\begin{array}{r}123.82 \\
93.96 \\
115.05\end{array}$ & $\begin{array}{l}108.53 \\
108.54 \\
108.53\end{array}$ & $\begin{array}{r}104.94 \\
84.29 \\
96.71\end{array}$ & $\begin{array}{r}41.80 \\
4.20 \\
4.20\end{array}$ & $\begin{array}{l}562.10 \\
420.60 \\
562.10\end{array}$ & $\begin{array}{r}92 \\
64 \\
156\end{array}$ \\
\hline 25.0 to 29.9 & $\begin{array}{c}1 \\
2 \\
1 \& 2\end{array}$ & $\begin{array}{l}133.27 \\
139.71 \\
136.12\end{array}$ & $\begin{array}{l}221.20 \\
219.40 \\
220.00\end{array}$ & $\begin{array}{l}157.97 \\
114.26 \\
113.25\end{array}$ & $\begin{array}{l}109.50 \\
127.00 \\
109.50\end{array}$ & $\begin{array}{l}332.90 \\
374.80 \\
374.80\end{array}$ & $\begin{array}{l}2 \\
4 \\
6\end{array}$ \\
\hline
\end{tabular}




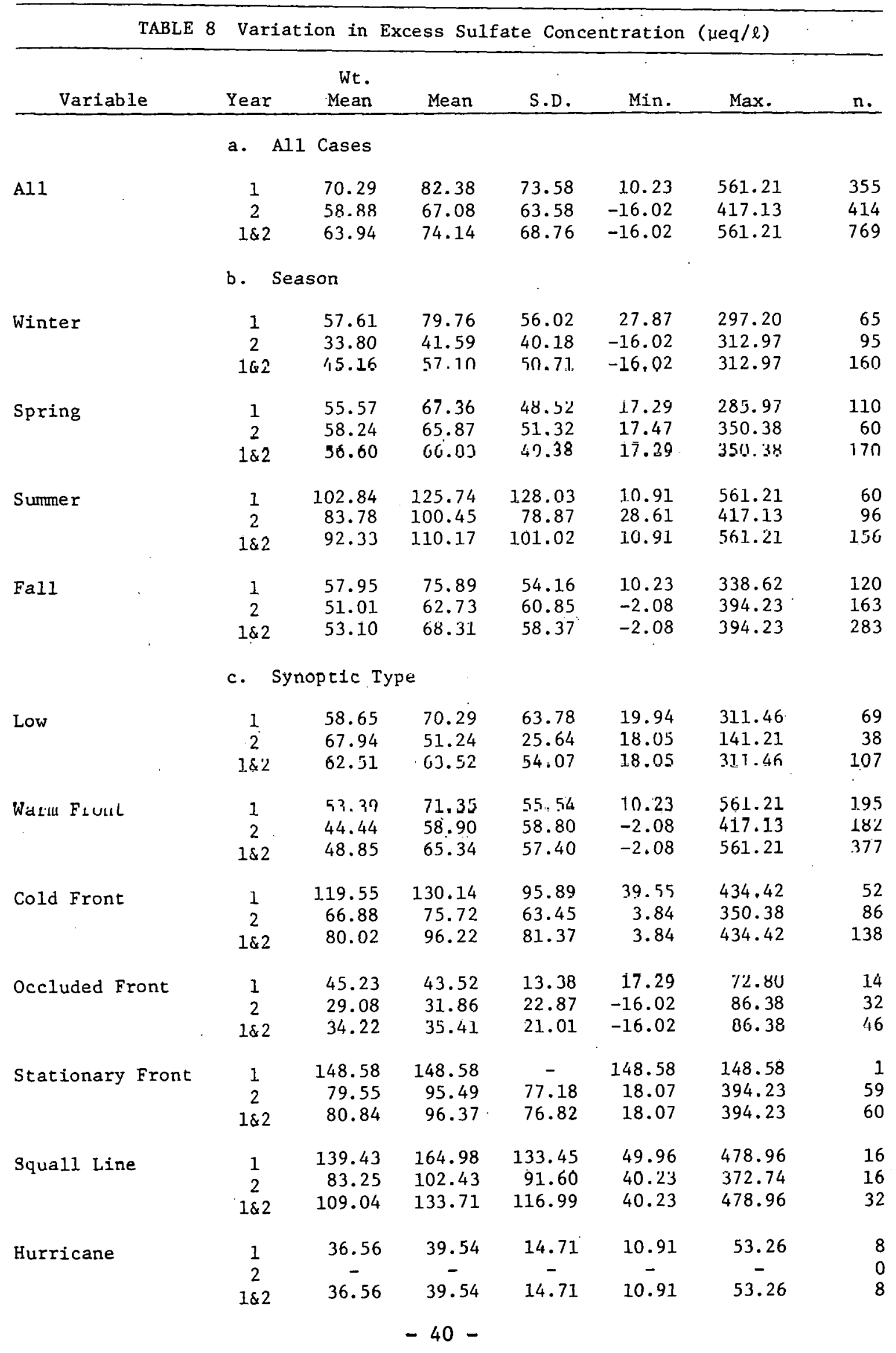


TABLE 8 (continued) Excess Sulfate

\begin{tabular}{|c|c|c|c|c|c|c|c|}
\hline Variable & Year & $\begin{array}{l}\text { Wt. } \\
\text { Mean }\end{array}$ & Mean & S.D. & Min. & Max: & $\mathrm{n}$. \\
\hline & \multicolumn{7}{|c|}{ d. Precipitation Type } \\
\hline Rain & $\begin{array}{c}1 \\
2 \\
1 \& 2\end{array}$ & $\begin{array}{l}53.62 \\
45.93 \\
49.37\end{array}$ & $\begin{array}{l}66.76 \\
57.71 \\
61.98\end{array}$ & $\begin{array}{l}55.74 \\
52.03 \\
53.96\end{array}$ & $\begin{array}{l}10.23 \\
-2.08 \\
-2.08\end{array}$ & $\begin{array}{l}561.21 \\
394.23 \\
561.21\end{array}$ & $\begin{array}{r}263 \\
294 \\
557\end{array}$ \\
\hline Rainshower & $\begin{array}{c}1 \\
2 \\
1 \& 2\end{array}$ & $\begin{array}{r}94.88 \\
120.57 \\
108.37\end{array}$ & $\begin{array}{l}147.07 \\
140.43 \\
143.40\end{array}$ & $\begin{array}{r}105.71 \\
94.97 \\
99.36\end{array}$ & $\begin{array}{l}39.33 \\
34.91 \\
34.91\end{array}$ & $\begin{array}{l}478.96 \\
417.13 \\
478.96\end{array}$ & $\begin{array}{l}38 \\
47 \\
85\end{array}$ \\
\hline Thundershower & $\begin{array}{c}1 \\
2 \\
1 \& 2\end{array}$ & $\begin{array}{r}113.36 \\
84.85 \\
97.24\end{array}$ & $\begin{array}{r}145.74 \\
87.71 \\
117.32\end{array}$ & $\begin{array}{r}111.65 \\
60.66 \\
94.10\end{array}$ & $\begin{array}{l}10.91 \\
20.35 \\
10.91\end{array}$ & $\begin{array}{l}369.36 \\
272.90 \\
369.36\end{array}$ & $\begin{array}{l}25 \\
24 \\
49\end{array}$ \\
\hline Mixed & $\begin{array}{c}1 \\
2 \\
1 \& 2\end{array}$ & $\begin{array}{r}70.24 \\
110.85 \\
82.98\end{array}$ & $\begin{array}{l}73.77 \\
88.81 \\
81.29\end{array}$ & $\begin{array}{l}21.49 \\
62.68 \\
42.71\end{array}$ & $\begin{array}{l}48.95 \\
18.62 \\
18.62\end{array}$ & $\begin{array}{r}86.26 \\
139.18 \\
139.18\end{array}$ & $\begin{array}{l}3 \\
3 \\
6\end{array}$ \\
\hline Sleet & $\begin{array}{c}1 \\
2 \\
1 \& 2\end{array}$ & $\begin{array}{c}- \\
37.98 \\
37.98\end{array}$ & $\begin{array}{c}- \\
54.30 \\
54.30\end{array}$ & $\begin{array}{c}- \\
40.47 \\
40.47\end{array}$ & $\begin{array}{c}- \\
14.63 \\
14.63\end{array}$ & $\begin{array}{c}- \\
112.74 \\
112.74\end{array}$ & $\begin{array}{l}0 \\
6 \\
6\end{array}$ \\
\hline Snow & $\begin{array}{c}1 \\
2 \\
1 \& 2\end{array}$ & $\begin{array}{l}81.71 \\
32.98 \\
52.67\end{array}$ & $\begin{array}{l}85.87 \\
37.67 \\
56.66\end{array}$ & $\begin{array}{l}50.95 \\
30.00 \\
45.84\end{array}$ & $\begin{array}{r}35.91 \\
-16.02 \\
-16.02\end{array}$ & $\begin{array}{l}271.06 \\
128.28 \\
271.06\end{array}$ & $\begin{array}{l}26 \\
40 \\
66\end{array}$ \\
\hline & e. & cipitat & on Rate & $(\mathrm{mm} / \mathrm{hr})$ & & & \\
\hline $\begin{array}{c}0.0-1.0 \\
1\end{array}$ & $\begin{array}{c}1 \\
2 \\
1 \& 2\end{array}$ & $\begin{array}{l}98.42 \\
78.48 \\
87.17\end{array}$ & $\begin{array}{r}101.41 \\
77.96 \\
89.14\end{array}$ & $\begin{array}{l}87.70 \\
74.90 \\
82.00\end{array}$ & $\begin{array}{r}10.23 \\
-16.02 \\
-16.02\end{array}$ & $\begin{array}{l}561.21 \\
417.13 \\
561.21\end{array}$ & $\begin{array}{l}186 \\
204 \\
390\end{array}$ \\
\hline $1.1-2.5$ & $\begin{array}{c}1 \\
2 \\
1 \& 2\end{array}$ & $\begin{array}{l}61.39 \\
60.07 \\
60.67\end{array}$ & $\begin{array}{l}63.16 \\
61.53 \\
62.26\end{array}$ & $\begin{array}{l}48.35 \\
54.78 \\
51.86\end{array}$ & $\begin{array}{r}14.75 \\
7.65 \\
7.65\end{array}$ & $\begin{array}{l}283.15 \\
300.26 \\
300.26\end{array}$ & $\begin{array}{r}98 \\
119 \\
217\end{array}$ \\
\hline $2.6-0.0$ & $\begin{array}{c}1 \\
2 \\
1 \& 2\end{array}$ & $\begin{array}{l}49.64 \\
50.31 \\
50.02\end{array}$ & $\begin{array}{l}51.01 \\
50.42 \\
50.67\end{array}$ & $\begin{array}{l}23.35 \\
36.87 \\
31.68\end{array}$ & $\begin{array}{l}29.39 \\
-2.08 \\
-2.08\end{array}$ & $\begin{array}{l}164.08 \\
168.58 \\
168.58\end{array}$ & $\begin{array}{r}17 \\
63 \\
110\end{array}$ \\
\hline $6.1-12.0$ & $\begin{array}{c}1 \\
2 \\
1 \& 2\end{array}$ & $\begin{array}{l}69.11 \\
44.18 \\
54.87\end{array}$ & $\begin{array}{l}69.45 \\
43.44 \\
54.39\end{array}$ & $\begin{array}{l}65.37 \\
29.09 \\
48.80\end{array}$ & $\begin{array}{r}10.91 \\
7.13 \\
7.13\end{array}$ & $\begin{array}{l}266.96 \\
105.83 \\
266.96\end{array}$ & $\begin{array}{l}16 \\
22 \\
38\end{array}$ \\
\hline$>12.0$ & $\begin{array}{c}1 \\
2 \\
1 \& 2\end{array}$ & $\begin{array}{l}91.48 \\
72.59 \\
81.76\end{array}$ & $\begin{array}{l}85.59 \\
68.97 \\
78.46\end{array}$ & $\begin{array}{l}5.9 .98 \\
58.36 \\
57.61\end{array}$ & $\begin{array}{l}38.33 \\
20.35 \\
20.35\end{array}$ & $\begin{array}{l}205.00 \\
174.87 \\
205.00\end{array}$ & $\begin{array}{r}8 \\
6 \\
14\end{array}$ \\
\hline
\end{tabular}




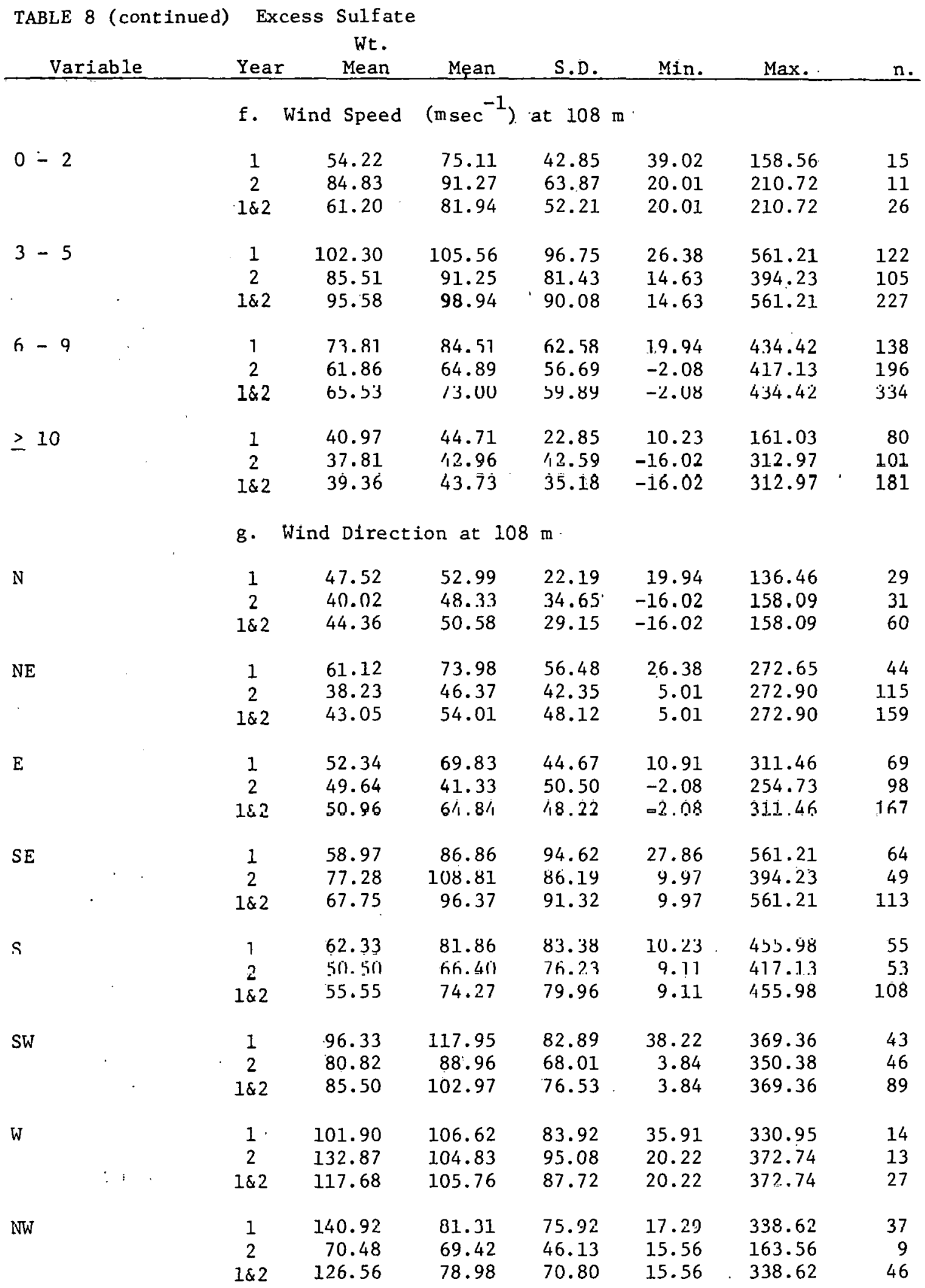


TABLE 8 (continued) Excess Sulfate

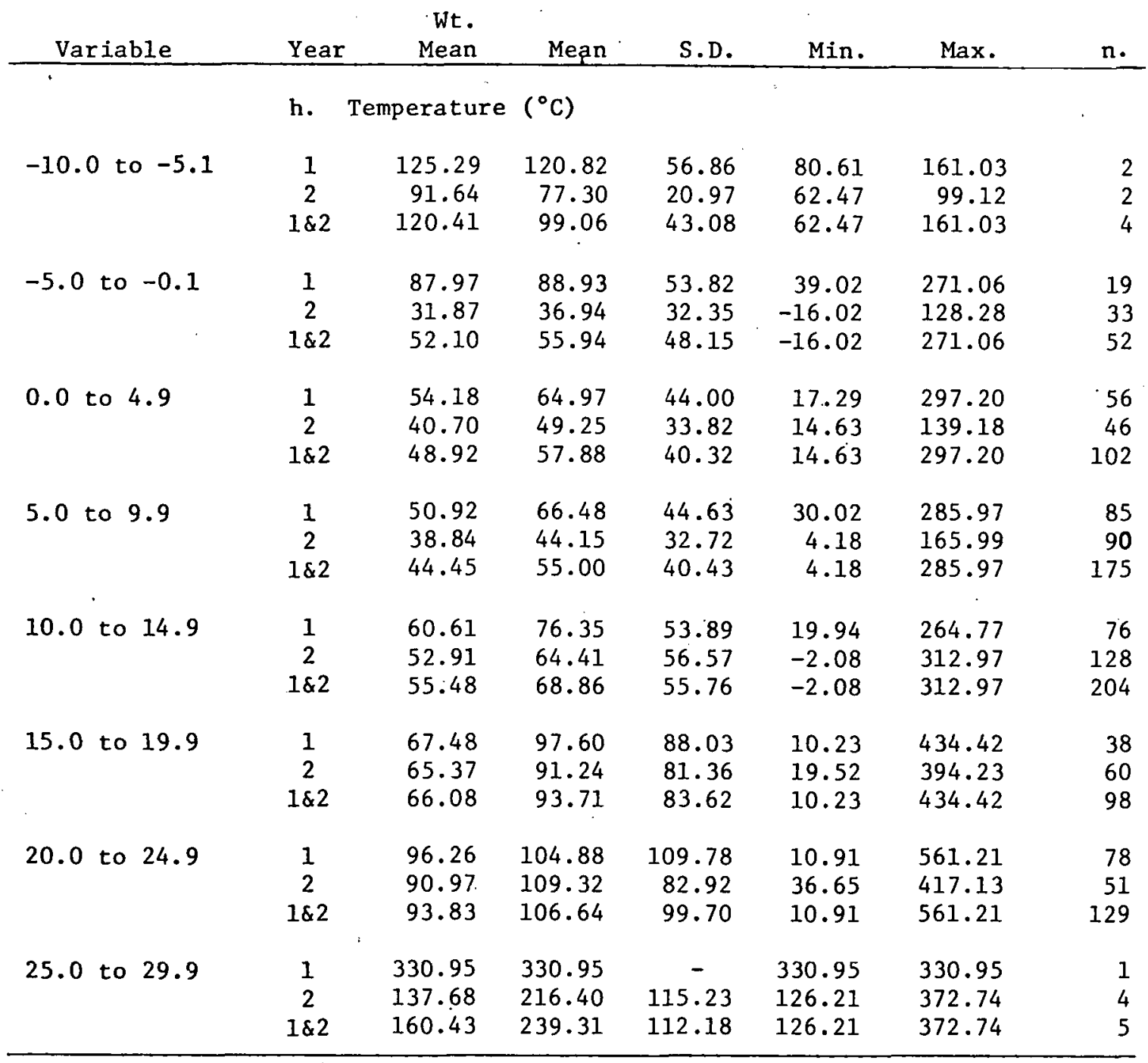




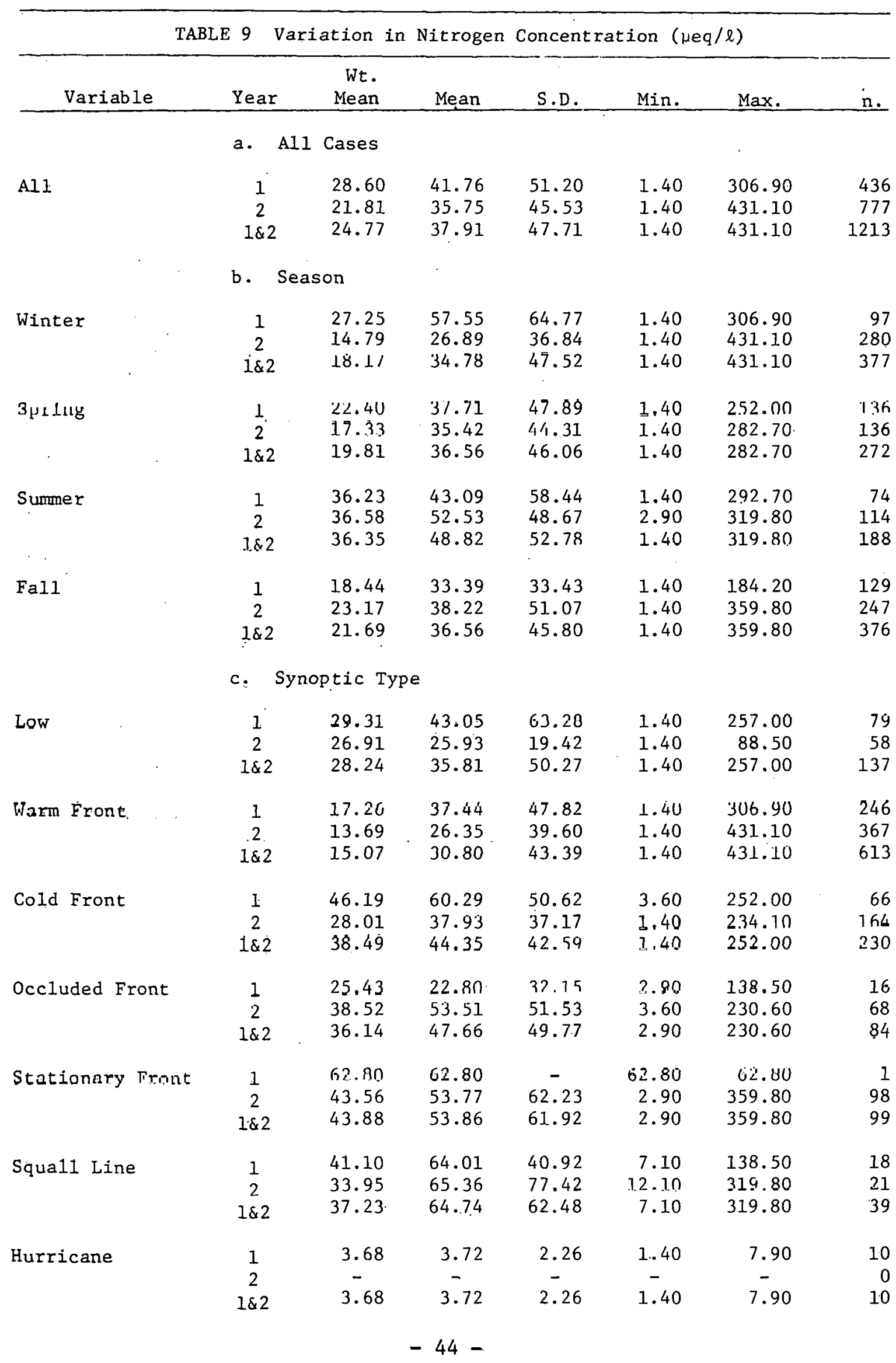


TABLE 9 (continued) Nitrogen

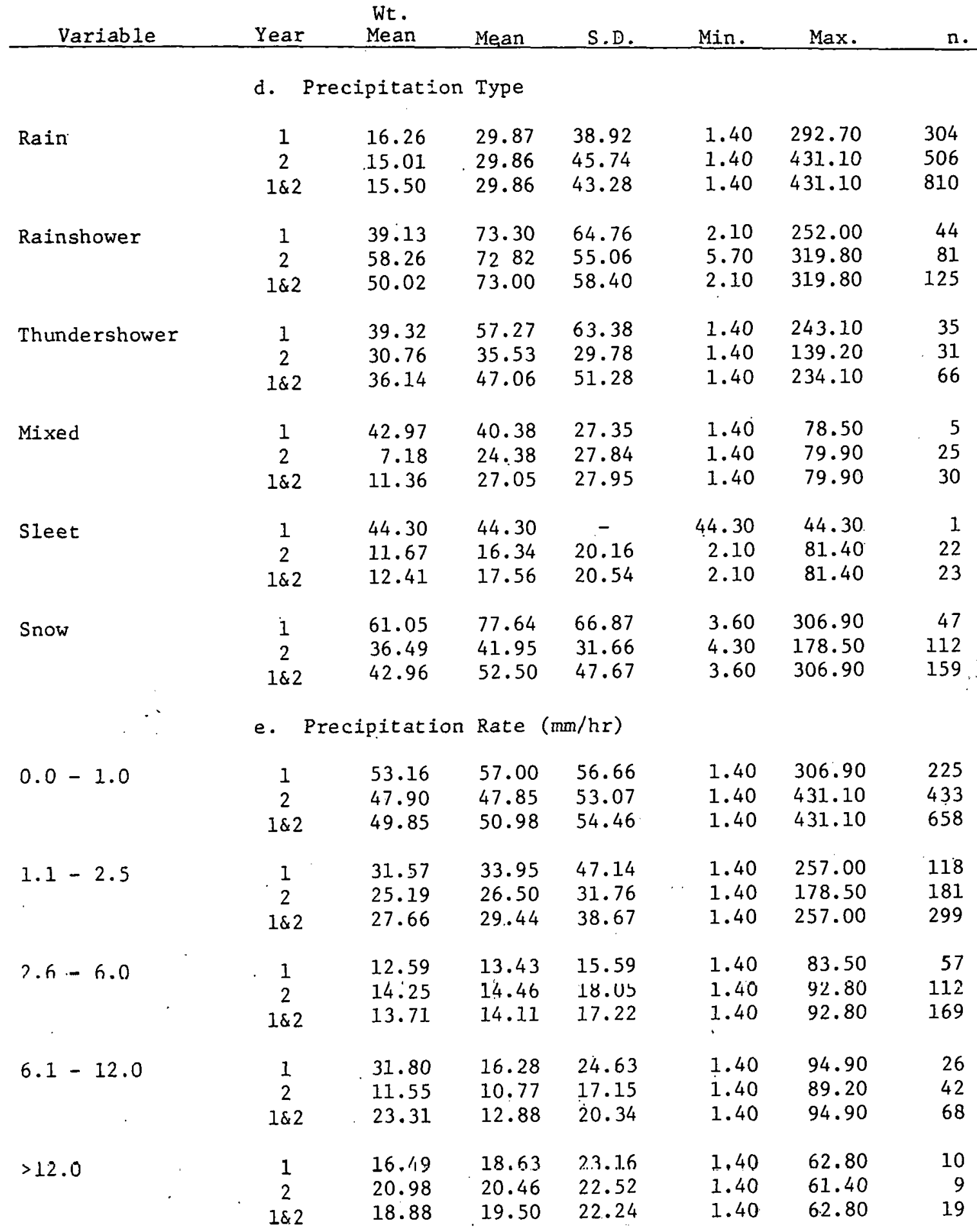


TABLE 9 (continued) Nitrogen

Wt.

\begin{tabular}{|c|c|c|c|c|c|c|c|}
\hline Variable & Year & Mean & Mean & S.D. & Min. & Max. & $\mathrm{n}$. \\
\hline & f. & Speed & (msec $^{-1}$ & at $108 \mathrm{Im}$ & & & \\
\hline $0-2$ & $\begin{array}{c}1 \\
2 \\
1 \& 2\end{array}$ & $\begin{array}{l}26.76 \\
52.91 \\
37.85\end{array}$ & $\begin{array}{l}59.12 \\
64.70 \\
62.70\end{array}$ & $\begin{array}{l}69.55 \\
60.77 \\
63.44\end{array}$ & $\begin{array}{l}1.40 \\
8.60 \\
1.40\end{array}$ & $\begin{array}{l}246.30 \\
282.70 \\
282.70\end{array}$ & $\begin{array}{l}19 \\
34 \\
53\end{array}$ \\
\hline $3-5$ & $\begin{array}{c}1 \\
2 \\
1 \& 2\end{array}$ & $\begin{array}{l}37.82 \\
33.88 \\
36.62\end{array}$ & $\begin{array}{l}46.96 \\
46.62 \\
46.76\end{array}$ & $\begin{array}{l}50.91 \\
48.95 \\
49.72\end{array}$ & $\begin{array}{l}1.40 \\
1.40 \\
1.40\end{array}$ & $\begin{array}{l}292.70 \\
359.80 \\
359.80\end{array}$ & $\begin{array}{l}163 \\
227 \\
390\end{array}$ \\
\hline $6-9$ & $\begin{array}{c}1 \\
2 \\
1 \& 2\end{array}$ & $\begin{array}{l}32.86 \\
23.90 \\
26.21\end{array}$ & $\begin{array}{l}47.43 \\
34.35 \\
38.68\end{array}$ & $\begin{array}{l}53.44 \\
47.22 \\
49.70\end{array}$ & $\begin{array}{l}1.40 \\
1.40 \\
1.40\end{array}$ & $\begin{array}{l}306.90 \\
431.10 \\
431.10\end{array}$ & $\begin{array}{l}165 \\
333 \\
498\end{array}$ \\
\hline$\geq 10$ & $\begin{array}{c}1 \\
2 \\
1 \& 2\end{array}$ & $\begin{array}{r}8.19 \\
11.86 \\
10.37\end{array}$ & $\begin{array}{l}18.02 \\
18.74 \\
18.48\end{array}$ & $\begin{array}{l}33.60 \\
22.99 \\
27.21\end{array}$ & $\begin{array}{l}1.40 \\
1.40 \\
1.40\end{array}$ & $\begin{array}{l}214.10 \\
155.60 \\
214.1 U\end{array}$ & $\begin{array}{r}89 \\
159 \\
248\end{array}$ \\
\hline
\end{tabular}

g. Wind Direction at $108 \mathrm{~m}$

\begin{tabular}{|c|c|c|c|c|c|c|c|}
\hline $\mathrm{N}$ & $\begin{array}{c}1 \\
2 \\
1 \& 2\end{array}$ & $\begin{array}{l}27.13 \\
31.01 \\
28.89\end{array}$ & $\begin{array}{l}41.36 \\
41.52 \\
41.45\end{array}$ & $\begin{array}{l}38.06 \\
42.28 \\
40.29\end{array}$ & $\begin{array}{l}1.40 \\
6.40 \\
1.40\end{array}$ & $\begin{array}{l}173.50 \\
234.10 \\
234.10\end{array}$ & $\begin{array}{l}36 \\
48 \\
84\end{array}$ \\
\hline NE & $\begin{array}{c}1 \\
2 \\
1 \& 2\end{array}$ & $\begin{array}{l}20.52 \\
19.12 \\
19.33\end{array}$ & $\begin{array}{l}37.90 \\
30.55 \\
31.83\end{array}$ & $\begin{array}{l}43.34 \\
40.40 \\
40.96\end{array}$ & $\begin{array}{l}1.40 \\
1.40 \\
1.40\end{array}$ & $\begin{array}{l}196.30 \\
431.10 \\
431.10\end{array}$ & $\begin{array}{r}57 \\
269 \\
326\end{array}$ \\
\hline E & $\begin{array}{c}1 \\
2 \\
1 \& 2\end{array}$ & $\begin{array}{l}12.60 \\
16.71 \\
14.78\end{array}$ & $\begin{array}{l}28.85 \\
30.46 \\
2 \dot{9} .90\end{array}$ & $\begin{array}{l}45.01 \\
45.75 \\
42.41\end{array}$ & $\begin{array}{l}1.40 \\
1.40 \\
1.40\end{array}$ & $\begin{array}{l}306.90 \\
359.80 \\
359.80\end{array}$ & $\begin{array}{r}83 \\
154 \\
237\end{array}$ \\
\hline $\mathrm{SE}$ & $\begin{array}{c}1 \\
2 \\
1 \& 2\end{array}$ & $\begin{array}{l}32.20 \\
23.24 \\
29.21\end{array}$ & $\begin{array}{l}38.87 \\
48.08 \\
43.95\end{array}$ & $\begin{array}{l}54.55 \\
60.19 \\
57.74\end{array}$ & $\begin{array}{l}1.40 \\
1.40 \\
1.40\end{array}$ & $\begin{array}{l}292.70 \\
253.40 \\
292.70\end{array}$ & $\begin{array}{r}74 \\
91 \\
165\end{array}$ \\
\hline$S$ & $\begin{array}{c}1 \\
2 \\
1 \& 2\end{array}$ & $\begin{array}{l}21.69 \\
13.95 \\
16.41\end{array}$ & $\begin{array}{l}38.22 \\
33.59 \\
35.46\end{array}$ & $\begin{array}{l}57.88 \\
45.93 \\
50.98\end{array}$ & $\begin{array}{l}2.10 \\
1.40 \\
1.40\end{array}$ & $\begin{array}{l}257.00 \\
207.00 \\
257.00\end{array}$ & $\begin{array}{r}62 \\
91 \\
153\end{array}$ \\
\hline SW & $\begin{array}{c}1 \\
2 \\
1 \& 2\end{array}$ & $\begin{array}{l}56.64 \\
36.68 \\
42.58\end{array}$ & $\begin{array}{l}74.08 \\
41.75 \\
54.54\end{array}$ & $\begin{array}{l}56.34 \\
36.08 \\
47.69\end{array}$ & $\begin{array}{l}2.10 \\
1.40 \\
1.40\end{array}$ & $\begin{array}{l}234.10 \\
148.50 \\
234.10\end{array}$ & $\begin{array}{r}51 \\
78 \\
129\end{array}$ \\
\hline $\mathrm{W}$ & $\begin{array}{c}1 \\
2 \\
1 \& 2\end{array}$ & $\begin{array}{l}47.60 \\
50.41 \\
49.10\end{array}$ & $\begin{array}{l}52.07 \\
47.18 \\
49.17\end{array}$ & $\begin{array}{l}49.31 \\
56.64 \\
53.34\end{array}$ & $\begin{array}{r}13.60 \\
2.10 \\
2.10\end{array}$ & $\begin{array}{l}214.10 \\
319.80 \\
319.80\end{array}$ & $\begin{array}{l}22 \\
32 \\
54\end{array}$ \\
\hline NW & $\begin{array}{c}1 \\
2 \\
1 \& 2\end{array}$ & $\begin{array}{l}42.99 \\
36.20 \\
41.73\end{array}$ & $\begin{array}{l}39.08 \\
48.23 \\
41.05\end{array}$ & $\begin{array}{l}47.92 \\
33.56 \\
45.14\end{array}$ & $\begin{array}{l}3.60 \\
5.00 \\
3.60\end{array}$ & $\begin{array}{l}214.10 \\
118.50 \\
214.10\end{array}$ & $\begin{array}{l}51 \\
14 \\
65\end{array}$ \\
\hline
\end{tabular}


TABLE 9 (continued) Nitrogen

Wt.

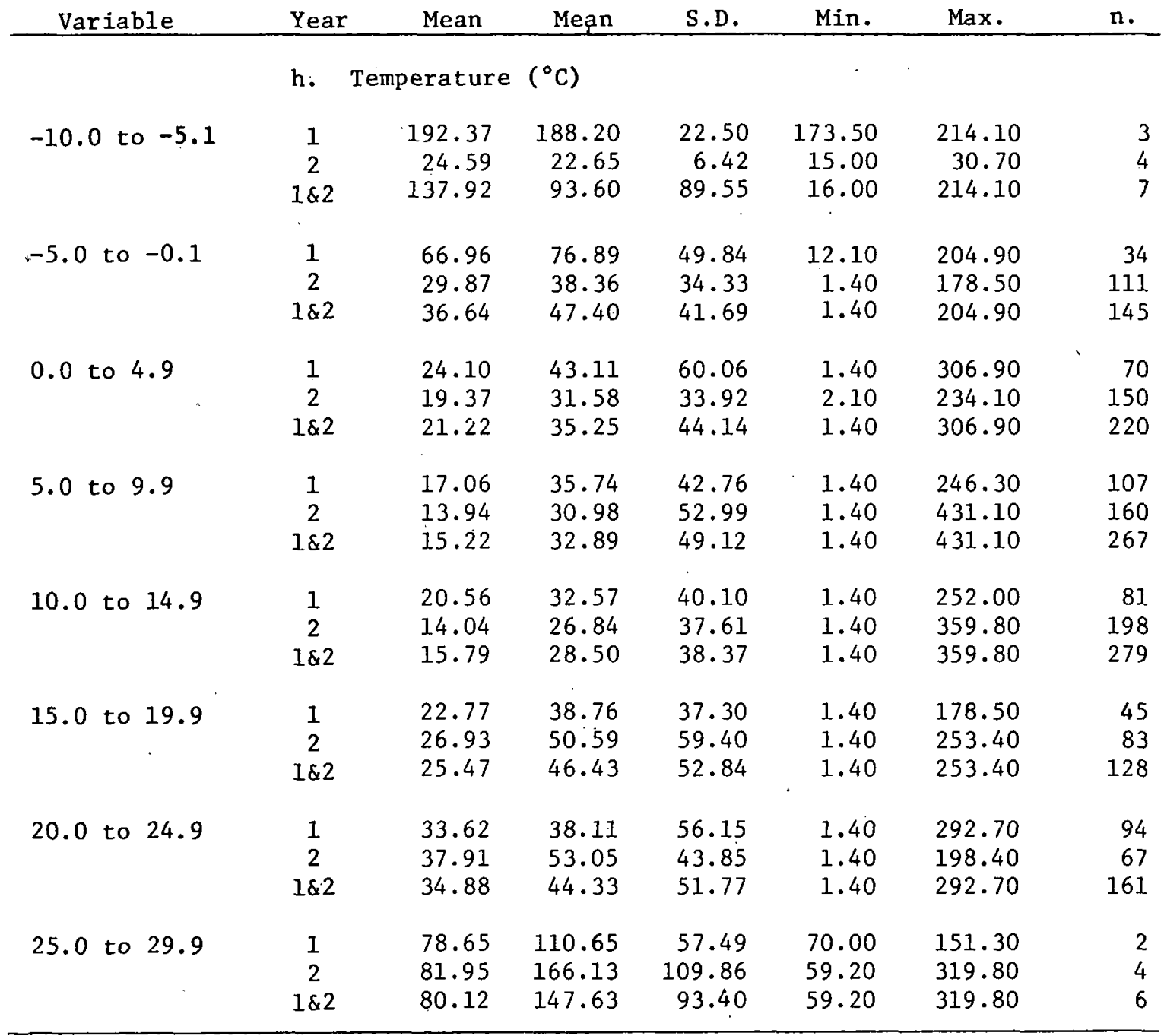




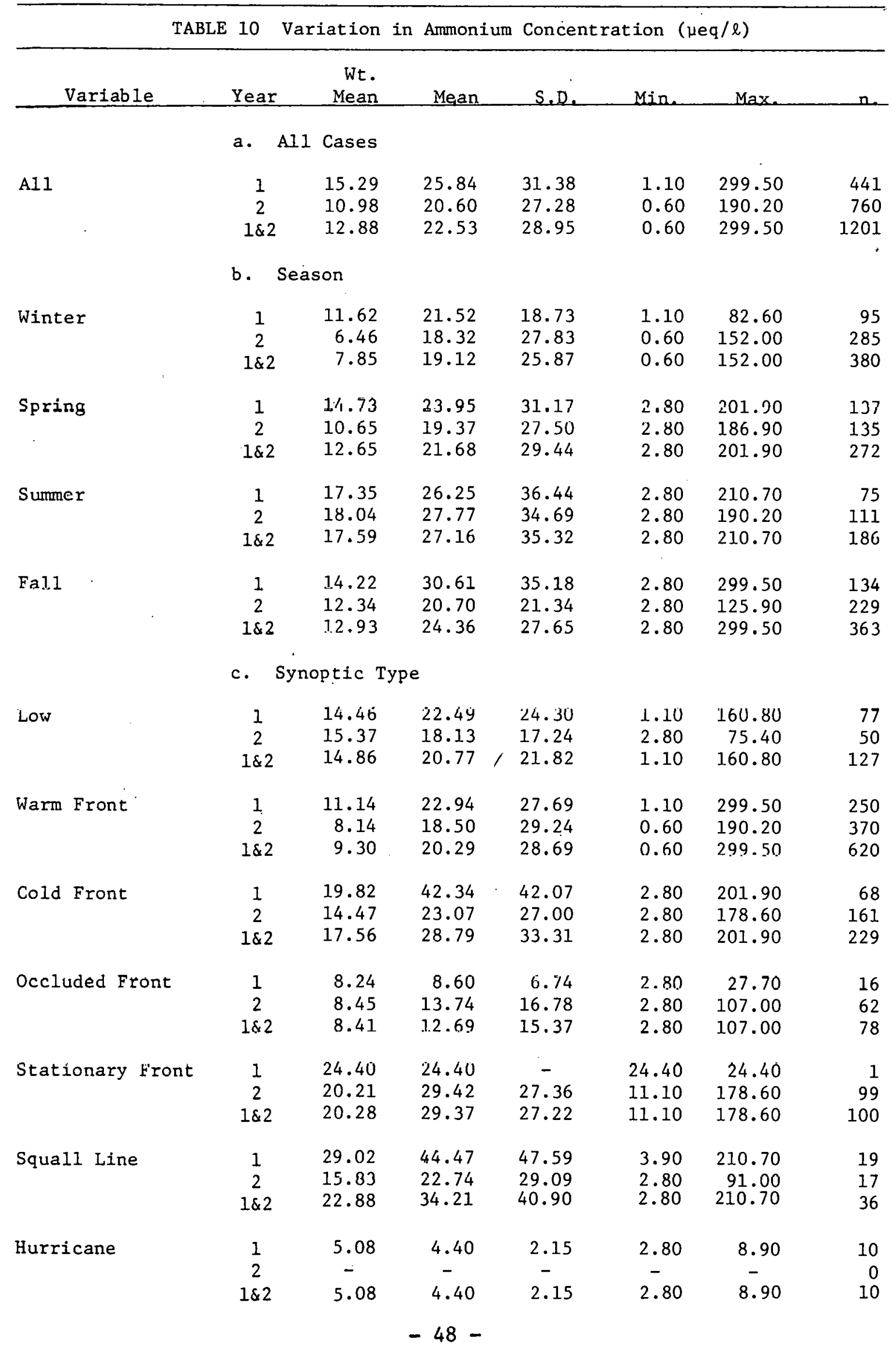


TABLE 10 (continued) Ammonium

Wt.

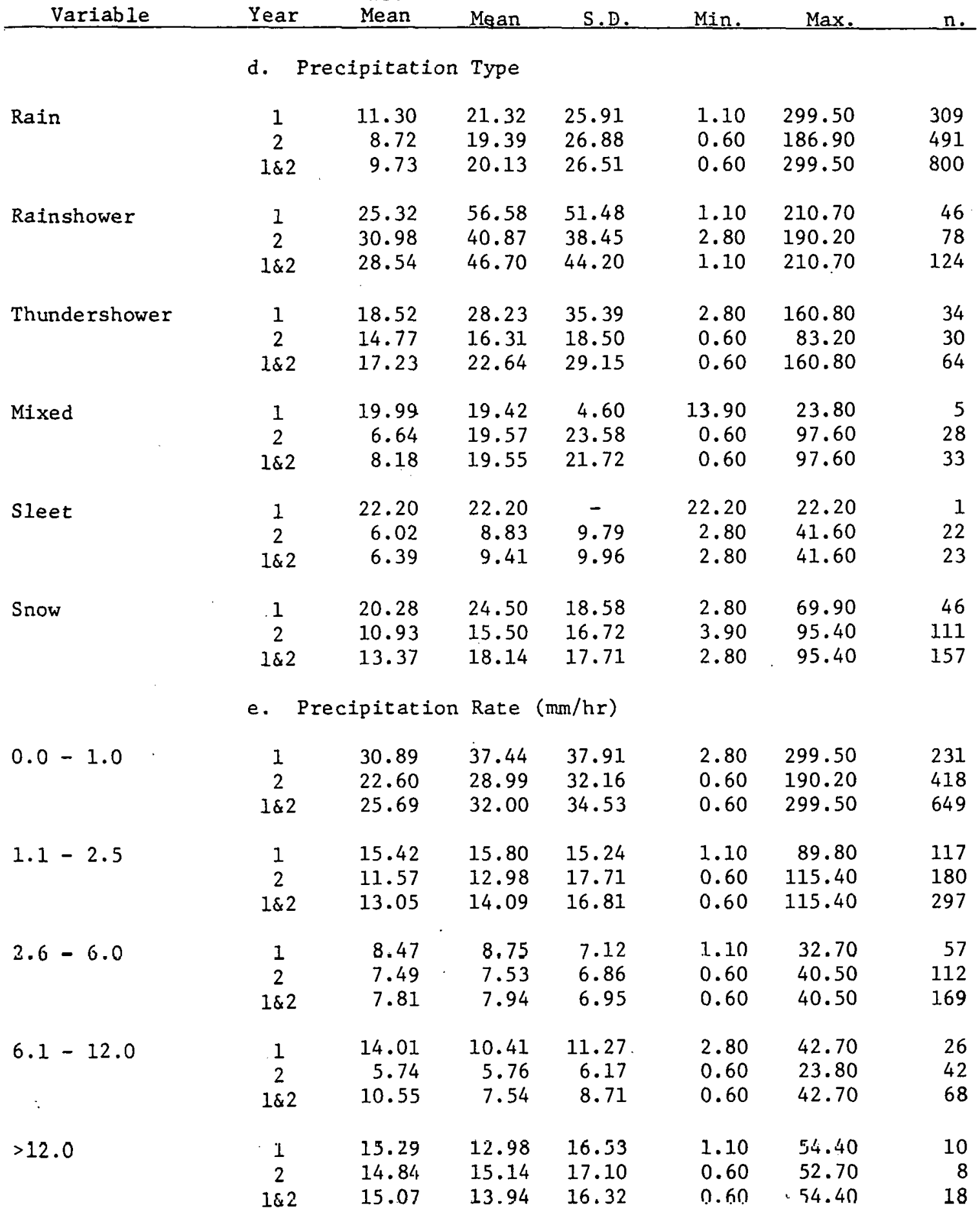




\begin{tabular}{|c|c|c|c|c|c|c|c|}
\hline Variable & Year & $\begin{array}{l}\text { Wt. } \\
\text { Mean }\end{array}$ & Mean & S.D. & Min. & Max. & $\mathrm{n}$. \\
\hline & f. & 1 Speed & (msec $^{-1}$ & at $108 \mathrm{~m}$ & & & \\
\hline $0-2$ & $\begin{array}{c}1 \\
2 \\
1 \& 2\end{array}$ & $\begin{array}{l}11.44 \\
24.08 \\
16.63\end{array}$ & $\begin{array}{l}25.52 \\
41.02 \\
34.64\end{array}$ & $\begin{array}{l}26.39 \\
38.36 \\
34.52\end{array}$ & $\begin{array}{l}2.80 \\
2.80 \\
2.80\end{array}$ & $\begin{array}{r}99.80 \\
186.90 \\
186.90\end{array}$ & $\begin{array}{l}21 \\
30 \\
51\end{array}$ \\
\hline $3-5$ & $\begin{array}{c}1 \\
2 \\
1 \& 2\end{array}$ & $\begin{array}{l}18.72 \\
18.74 \\
18.72\end{array}$ & $\begin{array}{l}29.36 \\
24.89 \\
26.80\end{array}$ & $\begin{array}{l}31.01 \\
26.68 \\
28.66\end{array}$ & $\begin{array}{l}2.80 \\
0.60 \\
0.60\end{array}$ & $\begin{array}{l}210.70 \\
178.60 \\
210.70\end{array}$ & $\begin{array}{l}165 \\
221 \\
386\end{array}$ \\
\hline $6-9$ & $\begin{array}{c}1 \\
2 \\
1 \& 2\end{array}$ & $\begin{array}{l}19.19 \\
11.33 \\
13.42\end{array}$ & $\begin{array}{l}29.93 \\
19.43 \\
22.97\end{array}$ & $\begin{array}{l}36.87 \\
25.99 \\
30.47\end{array}$ & $\begin{array}{l}1.10 \\
0.60 \\
0.60\end{array}$ & $\begin{array}{l}299.50 \\
190.20 \\
299.50\end{array}$ & $\begin{array}{l}165 \\
324 \\
489\end{array}$ \\
\hline$\geq 10$ & $\begin{array}{c}1 \\
2 \\
1 \& 2\end{array}$ & $\begin{array}{l}6.24 \\
6.38 \\
6.32\end{array}$ & $\begin{array}{l}11.98 \\
15.05 \\
13.96\end{array}$ & $\begin{array}{l}13.70 \\
27.15 \\
23.30\end{array}$ & $\begin{array}{l}1.10 \\
0.60 \\
0.60\end{array}$ & $\begin{array}{r}66.60 \\
152.00 \\
152.00\end{array}$ & $\begin{array}{r}90 \\
164 \\
254\end{array}$ \\
\hline
\end{tabular}

g. Wind Direction at $108 \mathrm{~m}$

\begin{tabular}{|c|c|c|c|c|c|c|c|}
\hline $\mathrm{N}$ & $\begin{array}{c}1 \\
2 \\
1 \& 2\end{array}$ & $\begin{array}{l}13.98 \\
10.21 \\
12.28\end{array}$ & $\begin{array}{l}19.16 \\
15.93 \\
17.35\end{array}$ & $\begin{array}{l}11.77 \\
19.56 \\
16.58\end{array}$ & $\begin{array}{l}2.80 \\
2.80 \\
2.80\end{array}$ & $\begin{array}{r}56.00 \\
100.40 \\
100.40\end{array}$ & $\begin{array}{l}36 \\
46 \\
82\end{array}$ \\
\hline $\mathrm{NE}$ & $\begin{array}{c}1 \\
2 \\
1 \& 2\end{array}$ & $\begin{array}{r}12.40 \\
9.49 \\
9.93\end{array}$ & $\begin{array}{l}24.30 \\
18.67 \\
19.69\end{array}$ & $\begin{array}{l}24.21 \\
24.71 \\
24.68\end{array}$ & $\begin{array}{l}2.80 \\
2.80 \\
2.80\end{array}$ & $\begin{array}{r}99.80 \\
178.60 \\
178.60\end{array}$ & $\begin{array}{r}58 \\
263 \\
321\end{array}$ \\
\hline$E$ & $\begin{array}{c}1 \\
2 \\
1 \& 2\end{array}$ & $\begin{array}{r}9.84 \\
11.08 \\
10.50\end{array}$ & $\begin{array}{l}19.49 \\
20.24 \\
19.98\end{array}$ & $\begin{array}{l}22.64 \\
28.22 \\
26.35\end{array}$ & $\begin{array}{l}2.80 \\
2.80 \\
2.80\end{array}$ & $\begin{array}{l}160.80 \\
186.90 \\
186.90\end{array}$ & $\begin{array}{r}82 \\
152 \\
234\end{array}$ \\
\hline$S E$ & $\begin{array}{c}1 \\
2 \\
1 \& 2\end{array}$ & $\begin{array}{l}14.25 \\
10.49 \\
13.00\end{array}$ & $\begin{array}{l}21.45 \\
22.76 \\
22.15\end{array}$ & $\begin{array}{l}26.89 \\
32.00 \\
29.63\end{array}$ & $\begin{array}{l}2.20 \\
0.60 \\
0.60\end{array}$ & $\begin{array}{l}201.90 \\
190.20 \\
201.90\end{array}$ & $\begin{array}{r}75 \\
85 \\
160\end{array}$ \\
\hline 3 & $\begin{array}{c}1 \\
2 \\
1 \& 2\end{array}$ & $\begin{array}{r}14.88 \\
8.04 \\
10.22\end{array}$ & $\begin{array}{l}33.03 \\
22.99 \\
27.06\end{array}$ & $\begin{array}{l}42.78 \\
32.93 \\
37.43\end{array}$ & $\begin{array}{l}1.10 \\
0.60 \\
0.60\end{array}$ & $\begin{array}{l}299.50 \\
152.00 \\
299.50\end{array}$ & $\begin{array}{r}64 \\
94 \\
158\end{array}$ \\
\hline SW & $\begin{array}{c}1 \\
2 \\
1 \& 2\end{array}$ & $\begin{array}{l}27.44 \\
14.09 \\
18.57\end{array}$ & $\begin{array}{l}45.94 \\
21.74 \\
31.89\end{array}$ & $\begin{array}{l}45.48 \\
26.01 \\
37.31\end{array}$ & $\begin{array}{l}1.10 \\
0.60 \\
0.60\end{array}$ & $\begin{array}{l}210.70 \\
178.60 \\
210.70\end{array}$ & $\begin{array}{r}52 \\
72 \\
124\end{array}$ \\
\hline $\mathrm{W}$ & $\begin{array}{c}1 \\
2 \\
1 \& 2\end{array}$ & $\begin{array}{l}22.44 \\
31.65 \\
27.38\end{array}$ & $\begin{array}{l}33.64 \\
26.22 \\
29.32\end{array}$ & $\begin{array}{l}37.48 \\
24.73 \\
30.61\end{array}$ & $\begin{array}{l}5.00 \\
2.80 \\
2.80\end{array}$ & $\begin{array}{l}143.10 \\
104.80 \\
143.10\end{array}$ & $\begin{array}{l}23 \\
32 \\
55\end{array}$ \\
\hline $\mathrm{NW}$ & $\begin{array}{c}1 \\
2 \\
1 \& 2\end{array}$ & $\begin{array}{l}27.44 \\
21.98 \\
26.40\end{array}$ & $\begin{array}{l}15.98 \\
27.42 \\
18.71\end{array}$ & $\begin{array}{l}14.91 \\
24.19 \\
18.04\end{array}$ & $\begin{array}{l}2.80 \\
2.80 \\
2.80\end{array}$ & $\begin{array}{l}69.90 \\
75.40 \\
75.40\end{array}$ & $\begin{array}{l}51 \\
16 \\
67\end{array}$ \\
\hline
\end{tabular}


TABLE 10 (continued) Ammonium

Wt.

\begin{tabular}{|c|c|c|c|c|c|c|c|}
\hline Variable & Year & Mean & Mean & S.D. & Min. & Max. & n. \\
\hline & h. & Temperatur & $\left({ }^{\circ} \mathrm{C}\right)$ & & & & \\
\hline-10.0 to -5.1 & $\begin{array}{c}1 \\
2 \\
1 \& 2\end{array}$ & $\begin{array}{l}32.71 \\
24.02 \\
29.23\end{array}$ & $\begin{array}{l}31.60 \\
24.52 \\
26.54\end{array}$ & $\begin{array}{l}14.14 \\
15.60 \\
14.41\end{array}$ & $\begin{array}{l}21.60 \\
10.00 \\
10.00\end{array}$ & $\begin{array}{l}41.60 \\
50.50 \\
50.50\end{array}$ & $\begin{array}{l}2 \\
5 \\
7\end{array}$ \\
\hline-5.0 to -0.1 & $\begin{array}{c}1 \\
2 \\
1 \& 2\end{array}$ & $\begin{array}{r}23.53 \\
9.53 \\
12.09\end{array}$ & $\begin{array}{l}26.61 \\
12.77 \\
16.13\end{array}$ & $\begin{array}{l}17.58 \\
13.16 \\
15.48\end{array}$ & $\begin{array}{l}2.80 \\
2.80 \\
2.80\end{array}$ & $\begin{array}{l}69.90 \\
75.40 \\
75.40\end{array}$ & $\begin{array}{r}34 \\
106 \\
140\end{array}$ \\
\hline 0.0 to 4.9 & $\begin{array}{c}1 \\
2 \\
1 \& 2\end{array}$ & $\begin{array}{l}13.52 \\
12.26 \\
12.75\end{array}$ & $\begin{array}{l}18.79 \\
27.68 \\
24.98\end{array}$ & $\begin{array}{l}15.98 \\
31.14 \\
27.70\end{array}$ & $\begin{array}{l}2.80 \\
0.60 \\
0.60\end{array}$ & $\begin{array}{r}77.60 \\
150.30 \\
150.30\end{array}$ & $\begin{array}{r}68 \\
156 \\
224\end{array}$ \\
\hline 5.0 to 9.9 & $\begin{array}{c}1 \\
2 \\
1 \& 2\end{array}$ & $\begin{array}{r}10.89 \\
7.45 \\
8.86\end{array}$ & $\begin{array}{l}20.99 \\
15.22 \\
17.64\end{array}$ & $\begin{array}{l}21.65 \\
24.81 \\
23.67\end{array}$ & $\begin{array}{l}2.80 \\
0.60 \\
0.60\end{array}$ & $\begin{array}{l}143.10 \\
186.90 \\
186.90\end{array}$ & $\begin{array}{l}109 \\
151 \\
260\end{array}$ \\
\hline 10.0 to 14.9 & $\begin{array}{c}1 \\
2 \\
1 \& 2\end{array}$ & $\begin{array}{r}14.80 \\
8.77 \\
10.40\end{array}$ & $\begin{array}{l}27.37 \\
17.90 \\
20.78\end{array}$ & $\begin{array}{l}31.52 \\
24.26 \\
26.98\end{array}$ & $\begin{array}{l}1.10 \\
0.60 \\
0.60\end{array}$ & $\begin{array}{l}201.90 \\
152.00 \\
201.90\end{array}$ & $\begin{array}{r}86 \\
196 \\
282\end{array}$ \\
\hline 15.0 to 19.9 & $\begin{array}{c}1 \\
2 \\
1 \& 2\end{array}$ & $\begin{array}{l}12.28 \\
12.66 \\
12.53\end{array}$ & $\begin{array}{l}32.58 \\
22.83 \\
26.50\end{array}$ & $\begin{array}{l}38.68 \\
24.96 \\
31.05\end{array}$ & $\begin{array}{l}2.80 \\
2.80 \\
2.80\end{array}$ & $\begin{array}{l}194.10 \\
109.80 \\
194.10\end{array}$ & $\begin{array}{r}47 \\
78 \\
125\end{array}$ \\
\hline 20.0 to 24.9 & $\begin{array}{c}1 \\
2 \\
1 \& 2\end{array}$ & $\begin{array}{l}17.17 \\
19.01 \\
17.66\end{array}$ & $\begin{array}{l}30.47 \\
31.65 \\
30.95\end{array}$ & $\begin{array}{l}44.64 \\
40.22 \\
42.76\end{array}$ & $\begin{array}{l}2.80 \\
2.80 \\
2.80\end{array}$ & $\begin{array}{l}299.50 \\
190.20 \\
299.50\end{array}$ & $\begin{array}{r}93 \\
64 \\
157\end{array}$ \\
\hline 25.0 to 29.9 & $\begin{array}{c}1 \\
2 \\
1 \& 2\end{array}$ & $\begin{array}{l}37.68 \\
32.48 \\
35.37\end{array}$ & $\begin{array}{l}72.40 \\
62.95 \\
66.10\end{array}$ & $\begin{array}{l}62.37 \\
30.59 \\
36.92\end{array}$ & $\begin{array}{l}28.30 \\
19.40 \\
19.40\end{array}$ & $\begin{array}{r}116.50 \\
91.00 \\
116.50\end{array}$ & $\begin{array}{l}2 \\
4 \\
6\end{array}$ \\
\hline
\end{tabular}




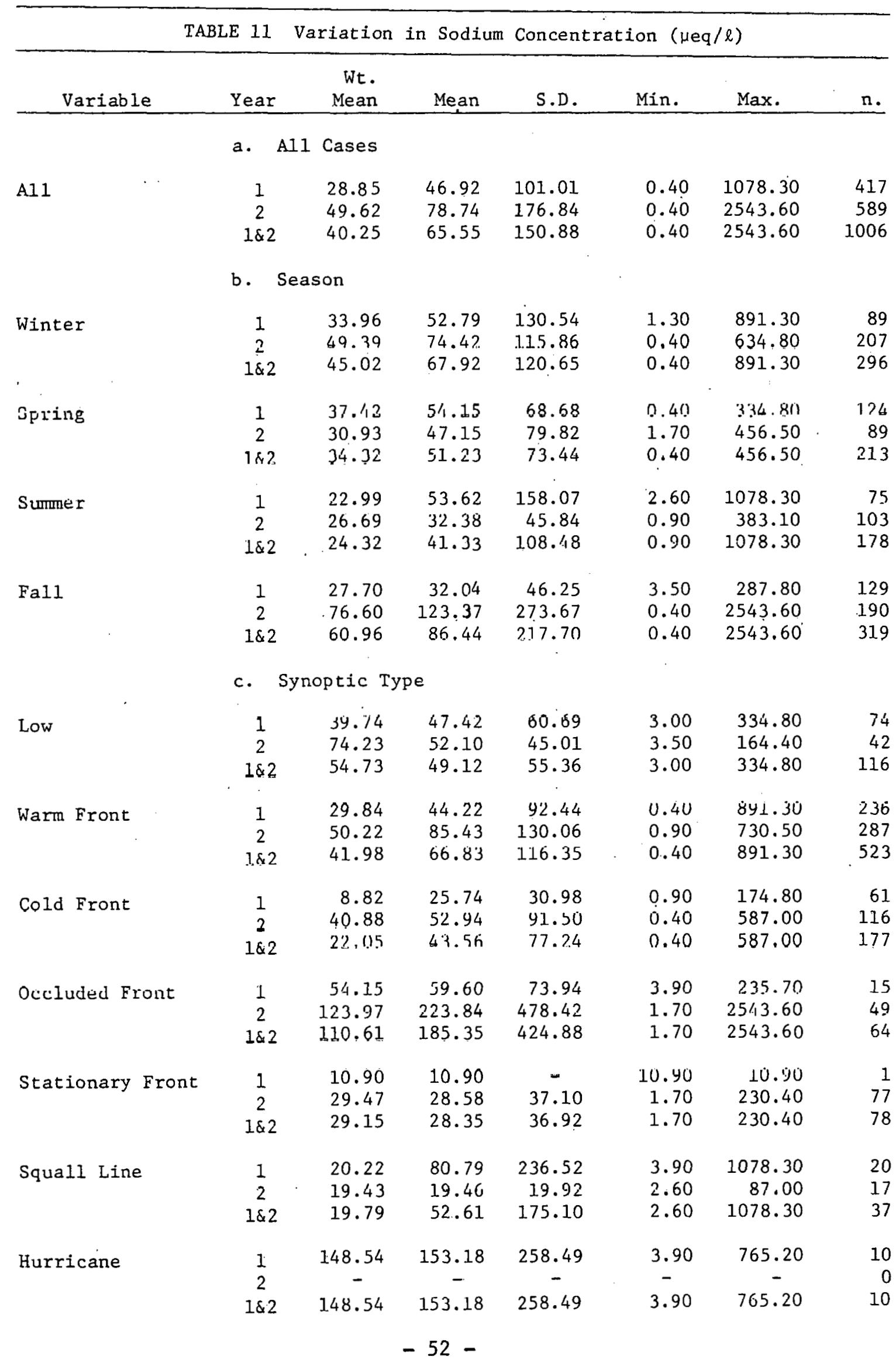


TABLE 11 (continued) Sodium

\begin{tabular}{|c|c|c|c|c|c|c|c|}
\hline Variable & Year & $\begin{array}{l}\text { Wt. } \\
\text { Mean }\end{array}$ & Mean: & S:D. & Min. & Max. & n. \\
\hline & \multicolumn{7}{|c|}{ Precipitation Type } \\
\hline Rain & $\begin{array}{c}1 \\
2 \\
1 \& 2\end{array}$ & $\begin{array}{l}35.20 \\
56.44 \\
47.84\end{array}$ & $\begin{array}{l}50.62 \\
91.40 \\
73.83\end{array}$ & $\begin{array}{r}97.43 \\
205.10 \\
168.54\end{array}$ & $\begin{array}{l}0.40 \\
0.40 \\
0.40\end{array}$ & $\begin{array}{r}891.30 \\
2543.60 \\
2543.60\end{array}$ & $\begin{array}{l}296 \\
391 \\
687\end{array}$ \\
\hline Rainshower & $\begin{array}{c}1 \\
2 \\
1 \& 2\end{array}$ & $\begin{array}{l}32.78 \\
39.22 \\
36.29\end{array}$ & $\begin{array}{l}55.43 \\
45.99 \\
50.32\end{array}$ & $\begin{array}{r}161.48 \\
73.61 \\
121.38\end{array}$ & $\begin{array}{l}0.90 \\
2.20 \\
0.90\end{array}$ & $\begin{array}{r}1078.30 \\
383.10 \\
1078.30\end{array}$ & $\begin{array}{l}44 \\
52 \\
96\end{array}$ \\
\hline Thundershower & $\begin{array}{c}1 \\
2 \\
1 \& 2\end{array}$ & $\begin{array}{l}21.35 \\
29.41 \\
24.32\end{array}$ & $\begin{array}{l}34.55 \\
28.77 \\
31.80\end{array}$ & $\begin{array}{l}83.56 \\
39.79 \\
65.98\end{array}$ & $\begin{array}{l}2.20 \\
0.90 \\
0.90\end{array}$ & $\begin{array}{l}482.60 \\
164.40 \\
482.60\end{array}$ & $\begin{array}{l}33 \\
30 \\
63\end{array}$ \\
\hline Mixed & $\begin{array}{c}1 \\
2 \\
1 \& 2\end{array}$ & $\begin{array}{r}6.14 \\
27.66 \\
25.07\end{array}$ & $\begin{array}{r}9.38 \\
38.43 \\
32.12\end{array}$ & $\begin{array}{l}10.09 \\
45.11 \\
41.7 .2\end{array}$ & $\begin{array}{l}1.30 \\
2.60 \\
.1 .30\end{array}$ & $\begin{array}{r}26.50 \\
195.70 \\
195.70\end{array}$ & $\begin{array}{r}5 \\
18 \\
23\end{array}$ \\
\hline Sleet & $\begin{array}{c}1 \\
2 \\
1 \& 2\end{array}$ & $\begin{array}{c}- \\
25.35 \\
25.35\end{array}$ & $\begin{array}{c}- \\
27.83 \\
27.83\end{array}$ & $\begin{array}{c}- \\
25.59 \\
25.59\end{array}$ & $\begin{array}{l}- \\
1.70 \\
1.70\end{array}$ & $\begin{array}{c}- \\
85.70 \\
85.70\end{array}$ & $\begin{array}{r}0 \\
21 \\
21\end{array}$ \\
\hline Snow & $\begin{array}{c}1 \\
2 \\
1 \& 2\end{array}$ & $\begin{array}{l}19.38 \\
69.68 \\
54.73\end{array}$ & $\begin{array}{l}24.57 \\
79.35 \\
60.93\end{array}$ & $\begin{array}{r}39.72 \\
132.58 \\
113.19\end{array}$ & $\begin{array}{l}2.60 \\
0.90 \\
0.90\end{array}$ & $\begin{array}{l}231.70 \\
634.80 \\
634.80\end{array}$ & $\begin{array}{r}39 \\
77 \\
116\end{array}$ \\
\hline & e. & cipita & n Rate & $\mathrm{mm} / \mathrm{hr}$ ) & & & \\
\hline $0.0-1.0$ & $\begin{array}{c}1 \\
2 \\
1 \& 2\end{array}$ & $\begin{array}{r}48.81 \\
113.92 \\
87.31\end{array}$ & $\begin{array}{r}52.37 \\
108.08 \\
83.66\end{array}$ & $\begin{array}{l}113.75 \\
242.72 \\
198.64\end{array}$ & $\begin{array}{l}0.90 \\
0.90 \\
0.90\end{array}$ & $\begin{array}{l}1078.30 \\
2543.60 \\
2543.60\end{array}$ & $\begin{array}{l}206 \\
264 \\
470\end{array}$ \\
\hline $1.1-2.5$ & $\begin{array}{c}1 \\
2 \\
1 \& 2\end{array}$ & $\begin{array}{l}47.65 \\
65.59 \\
58.43\end{array}$ & $\begin{array}{l}51.71 \\
70.83 \\
62.99\end{array}$ & $\begin{array}{l}103.31 \\
106.94 \\
105.71\end{array}$ & $\begin{array}{l}2.60 \\
0.40 \\
0.40\end{array}$ & $\begin{array}{l}891.30 \\
634.80 \\
891.30\end{array}$ & $\begin{array}{l}118 \\
170 \\
288\end{array}$ \\
\hline $2.6-6.0$ & $\begin{array}{c}1 \\
2 \\
1 \& 2\end{array}$ & $\begin{array}{l}26.17 \\
44.82 \\
38.43\end{array}$ & $\begin{array}{l}27.93 \\
44.84 \\
38.89\end{array}$ & $\begin{array}{l}35.03 \\
63.06 \\
55.33\end{array}$ & $\begin{array}{l}0.40 \\
0.40 \\
0.40\end{array}$ & $\begin{array}{l}190.40 \\
326.10 \\
326.10\end{array}$ & $\begin{array}{r}57 \\
105 \\
162\end{array}$ \\
\hline $6.1-12.0$ & $\begin{array}{c}1 \\
2 \\
1 \& 2\end{array}$ & $\begin{array}{l}22.55 \\
22.61 \\
22.58\end{array}$ & $\begin{array}{l}37.97 \\
23.79 \\
29.29\end{array}$ & $\begin{array}{l}95.53 \\
25.38 \\
62.41\end{array}$ & $\begin{array}{l}0.90 \\
0.90 \\
0.90\end{array}$ & $\begin{array}{l}482.60 \\
113.00 \\
482.60\end{array}$ & $\begin{array}{l}26 \\
41 \\
67\end{array}$ \\
\hline$>12.0$ & $\begin{array}{c}1 \\
2 \\
182\end{array}$ & $\begin{array}{r}9.65 \\
15.33 \\
12.68\end{array}$ & $\begin{array}{r}9.96 \\
13.52 \\
11.65\end{array}$ & $\begin{array}{r}7.72 \\
11.81 \\
9.75\end{array}$ & $\begin{array}{l}2.60 \\
0.90 \\
0.90\end{array}$ & $\begin{array}{l}28.70 \\
37.40 \\
37.40\end{array}$ & $\begin{array}{r}10 \\
9 \\
19\end{array}$ \\
\hline
\end{tabular}


TABLE 11 (continued) Sodium

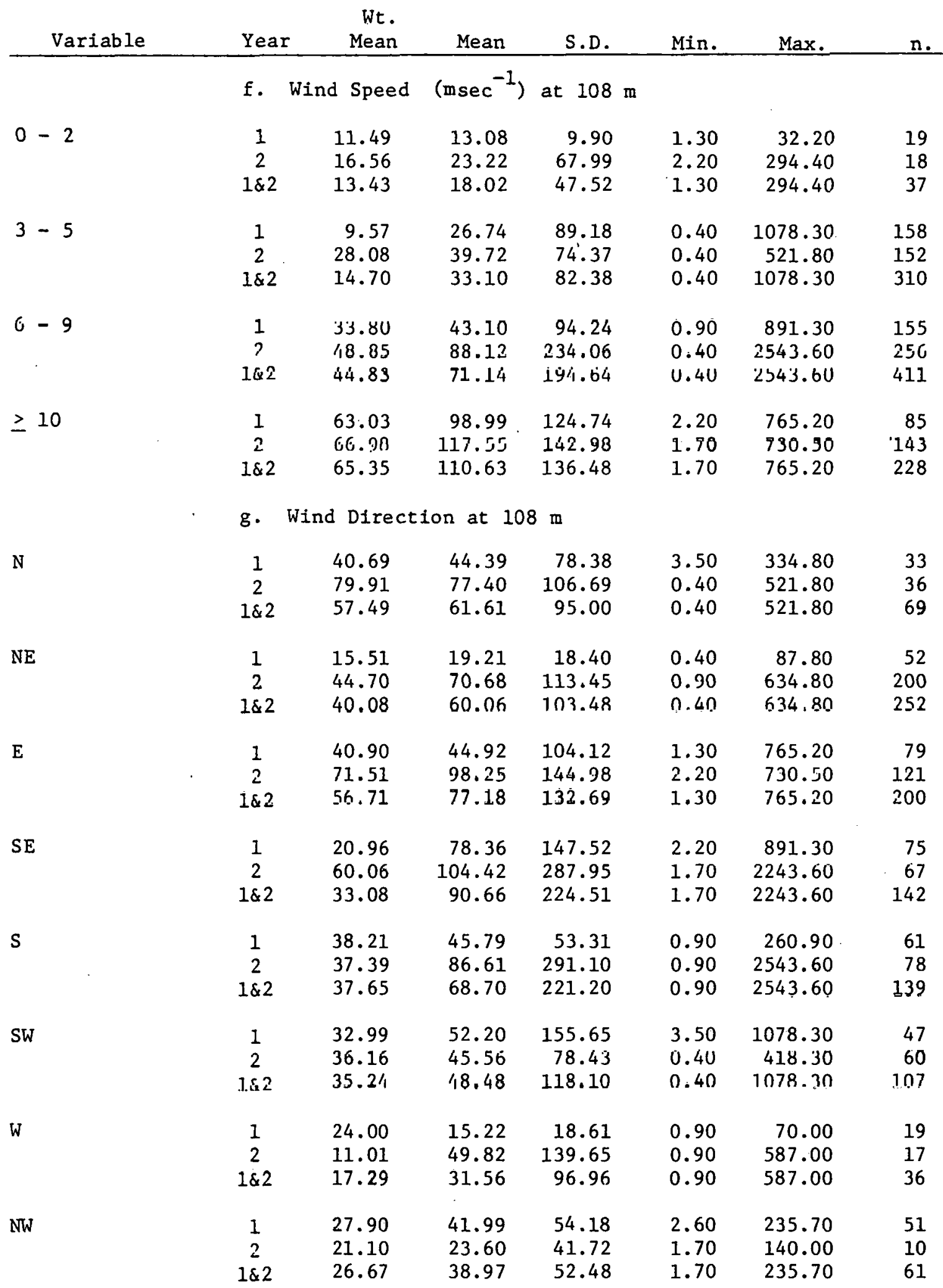


TABLE 11 (continued) Sodium

\begin{tabular}{|c|c|c|c|c|c|c|c|}
\hline Variable & Year & $\begin{array}{l}\text { Wt. } \\
\text { Mean }\end{array}$ & Mean & S.D. & Min. & Max. & $\mathrm{n}$. \\
\hline & \multicolumn{4}{|c|}{ Temperature $\left({ }^{\circ} \mathrm{C}\right)$} & & - & \\
\hline-10.0 to -5.1 & $\begin{array}{c}1 \\
2 \\
1 \& 2\end{array}$ & $\begin{array}{l}25.81 \\
39.52 \\
27.80\end{array}$ & $\begin{array}{r}25.45 \\
308.70 \\
167.08\end{array}$ & $\begin{array}{r}4.60 \\
393.58 \\
279.97\end{array}$ & $\begin{array}{l}22.20 \\
30.40 \\
22.20\end{array}$ & $\begin{array}{r}28.70 \\
587.00 \\
587.00\end{array}$ & $\begin{array}{l}2 \\
2 \\
4\end{array}$ \\
\hline-5.0 to -0.1 & $\begin{array}{c}1 \\
2 \\
1 \& 2\end{array}$ & $\begin{array}{l}24.49 \\
56.22 \\
50.28\end{array}$ & $\begin{array}{l}29.79 \\
58.82 \\
51.56\end{array}$ & $\begin{array}{l}47.14 \\
77.09 \\
71.70\end{array}$ & $\begin{array}{l}2.60 \\
0.90 \\
0.90\end{array}$ & $\begin{array}{l}231.70 \\
352.20 \\
352.20\end{array}$ & $\begin{array}{r}26 \\
78 \\
104\end{array}$ \\
\hline 0.0 to 4.9 & $\begin{array}{c}1 \\
2 \\
1 \& 2\end{array}$ & $\begin{array}{l}41.91 \\
35.76 \\
38.29\end{array}$ & $\begin{array}{l}56.92 \\
44.72 \\
49.69\end{array}$ & $\begin{array}{l}68.91 \\
94.20 \\
84.80\end{array}$ & $\begin{array}{l}1.30 \\
0.40 \\
0.40\end{array}$ & $\begin{array}{l}334.80 \\
634.80 \\
634.80\end{array}$ & $\begin{array}{r}68 \\
99 \\
167\end{array}$ \\
\hline 5.0 to 9.9 & $\begin{array}{c}1 \\
2 \\
1 \& 2\end{array}$ & $\begin{array}{l}24.57 \\
72.59 \\
52.61\end{array}$ & $\begin{array}{r}41.60 \\
142.04 \\
95.97\end{array}$ & $\begin{array}{l}101.20 \\
273.05 \\
217.69\end{array}$ & $\begin{array}{l}0.40 \\
0.90 \\
0.40\end{array}$ & $\begin{array}{r}891.30 \\
2543.60 \\
2543.60\end{array}$ & $\begin{array}{l}100 \\
118 \\
218\end{array}$ \\
\hline 10.0 to 14.9 & $\begin{array}{c}1 \\
2 \\
1 \& 2\end{array}$ & $\begin{array}{l}43.02 \\
58.46 \\
54.12\end{array}$ & $\begin{array}{l}51.89 . \\
91.27 \\
77.98\end{array}$ & $\begin{array}{r}99.28 \\
206.46 \\
178.41\end{array}$ & $\begin{array}{l}0.90 \\
0.90 \\
0.90\end{array}$ & $\begin{array}{r}669.60 \\
2243.60 \\
2243.60\end{array}$ & $\begin{array}{r}82 \\
161 \\
243\end{array}$ \\
\hline 15.0 to 19.9 & $\begin{array}{c}1 \\
2 \\
1 \& 2\end{array}$ & $\begin{array}{l}26.99 \\
29.34 \\
28.49\end{array}$ & $\begin{array}{l}33.48 \\
43.38 \\
39.41\end{array}$ & $\begin{array}{l}54.66 \\
57.59 \\
56.39\end{array}$ & $\begin{array}{l}3.50 \\
0.40 \\
0.40\end{array}$ & $\begin{array}{l}260.90 \\
294.40 \\
294.40\end{array}$ & $\begin{array}{r}45 \\
67 \\
112\end{array}$ \\
\hline 20.0 to 24.9 & $\begin{array}{c}1 \\
2 \\
1 \& 2\end{array}$ & $\begin{array}{l}24.22 \\
27.45 \\
25.16\end{array}$ & $\begin{array}{l}53.58 \\
38.07 \\
47.46\end{array}$ & $\begin{array}{r}144.17 \\
57.92 \\
117.87\end{array}$ & $\begin{array}{l}2.60 \\
0.90 \\
0.90\end{array}$ & $\begin{array}{r}1078.30 \\
383.10 \\
1078.30\end{array}$ & $\begin{array}{r}92 \\
60 \\
152\end{array}$ \\
\hline 25.0 to $29.9^{\circ}$ & $\begin{array}{c}1 \\
2 \\
1 \& 2\end{array}$ & $\begin{array}{r}5.20 \\
16.76 \\
10.33\end{array}$ & $\begin{array}{l}10.00 \\
24.80 \\
19.87\end{array}$ & $\begin{array}{r}8.63 \\
25.31 \\
21.40\end{array}$ & $\begin{array}{l}3.90 \\
6.50 \\
3.90\end{array}$ & $\begin{array}{l}16.10 \\
62.20 \\
62.20\end{array}$ & $\begin{array}{l}2 \\
4 \\
6\end{array}$ \\
\hline
\end{tabular}




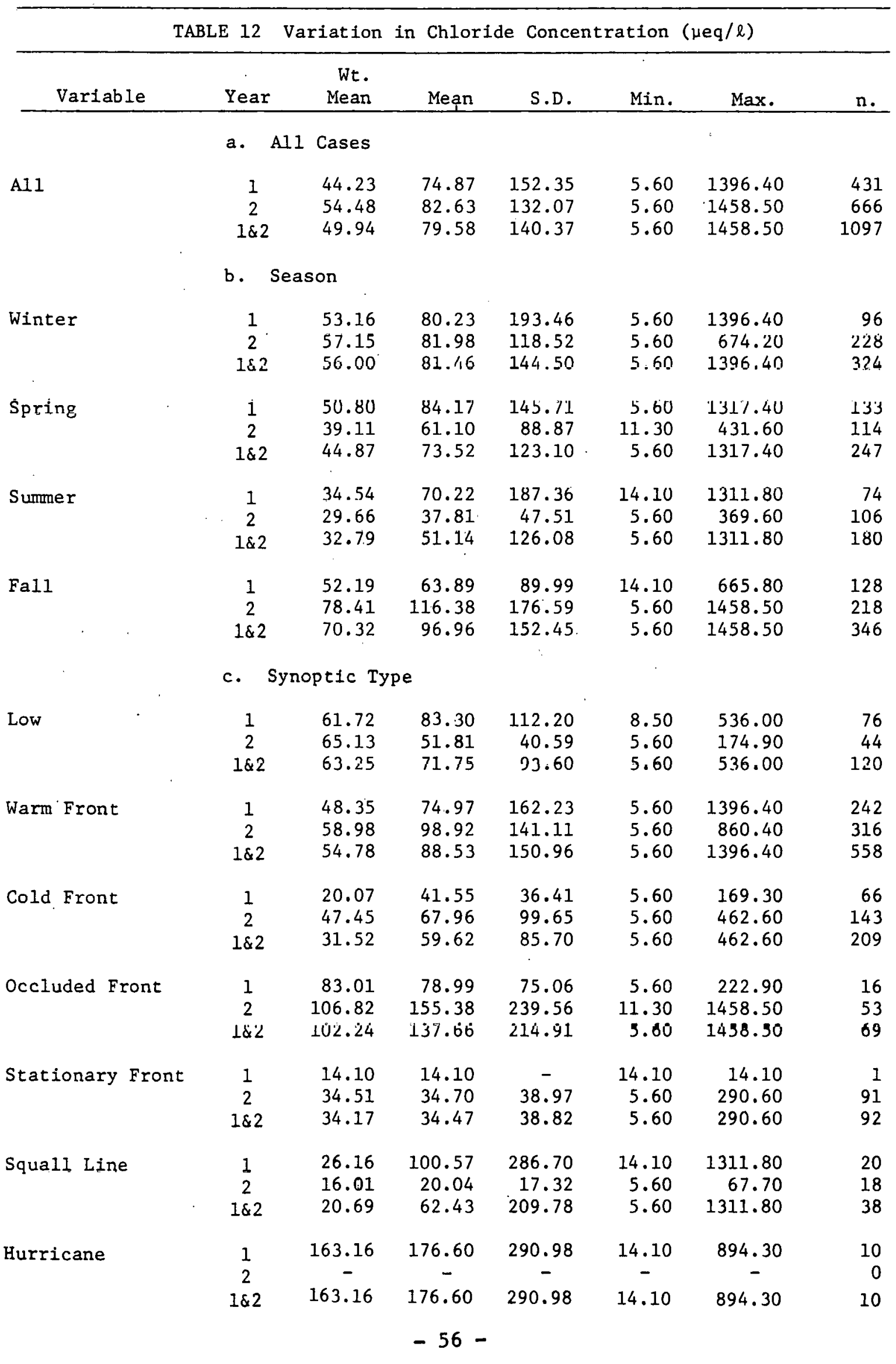


TABLE 12 (continued) Chloride

Wt.

Variable

Year

Mean

Mean

S.D.

Min.

Max.

n.

d. Precipitation Type

\begin{tabular}{|c|c|c|c|c|c|c|c|}
\hline Rain & $\begin{array}{c}1 \\
2 \\
1 \& 2\end{array}$ & $\begin{array}{l}54.30 \\
61.06 \\
58.39\end{array}$ & $\begin{array}{l}83.98 \\
95.32 \\
90.63\end{array}$ & $\begin{array}{l}161.50 \\
148.21 \\
153.84\end{array}$ & $\begin{array}{l}5.60 \\
5.60 \\
5.60\end{array}$ & $\begin{array}{l}1396.40 \\
1458.50 \\
1458.50\end{array}$ & $\begin{array}{l}303 \\
430 \\
733\end{array}$ \\
\hline Rainshower & $\begin{array}{c}1 \\
2 \\
1 \& 2\end{array}$ & $\begin{array}{l}47.19 \\
58.68 \\
53.56\end{array}$ & $\begin{array}{l}73.23 \\
64.08 \\
67.85\end{array}$ & $\begin{array}{r}187.80 \\
89.76 \\
138.15\end{array}$ & $\begin{array}{l}8.50 \\
5.60 \\
5.60\end{array}$ & $\begin{array}{r}1311.80 \\
442.90 \\
1311.80\end{array}$ & $\begin{array}{r}47 \\
67 \\
114\end{array}$ \\
\hline Thundershower & $\begin{array}{c}1 \\
2 \\
1 \& 2\end{array}$ & $\begin{array}{l}32.24 \\
26.60 \\
30.14\end{array}$ & $\begin{array}{l}48.12 \\
27.38 \\
38.23\end{array}$ & $\begin{array}{l}87.01 \\
31.35 \\
66.88\end{array}$ & $\begin{array}{l}5.60 \\
5.60 \\
5.60\end{array}$ & $\begin{array}{l}496.50 \\
129.80 \\
496.50\end{array}$ & $\begin{array}{l}34 \\
31 \\
65\end{array}$ \\
\hline Mixed & $\begin{array}{c}1 \\
2 \\
1 \& 2\end{array}$ & $\begin{array}{r}8.48 \\
44.18 \\
40.70\end{array}$ & $\begin{array}{r}8.47 \\
47.58 \\
43.07\end{array}$ & $\begin{array}{r}2.85 \\
73.76 \\
70.36\end{array}$ & $\begin{array}{l}5.60 \\
8.50 \\
5.60\end{array}$ & $\begin{array}{r}11.30 \\
301.80 \\
301.80\end{array}$ & $\begin{array}{r}3 \\
23 \\
26\end{array}$ \\
\hline Sleet & $\begin{array}{c}1 \\
2 \\
1 \& 2\end{array}$ & $\begin{array}{r}141.10 \\
38.95 \\
41.28\end{array}$ & $\begin{array}{r}141.10 \\
43.35 \\
47.60\end{array}$ & $\begin{array}{c}- \\
62.14 \\
64.04\end{array}$ & $\begin{array}{r}141.10 \\
8.50 \\
8.50\end{array}$ & $\begin{array}{l}141.10 \\
301.80 \\
301.80\end{array}$ & $\begin{array}{r}1 \\
22 \\
23\end{array}$ \\
\hline Snow & $\begin{array}{c}1 \\
2 \\
1 \& 2\end{array}$ & $\begin{array}{l}33.41 \\
74.49 \\
62.40\end{array}$ & $\begin{array}{l}36.75 \\
73.72 \\
62.03\end{array}$ & $\begin{array}{r}49.13 \\
112.15 \\
98.08\end{array}$ & $\begin{array}{l}8.50 \\
8.50 \\
8.50\end{array}$ & $\begin{array}{l}265.20 \\
674.20 \\
674.20\end{array}$ & $\begin{array}{r}43 \\
93 \\
136\end{array}$ \\
\hline & e. & ipitat & Rate & $(\mathrm{mm} / \mathrm{hr})$ & & & \\
\hline $0.0-1.0$ & $\begin{array}{c}1 \\
2 \\
1 \& 2\end{array}$ & $\begin{array}{r}75.46 \\
107.04 \\
94.56\end{array}$ & $\begin{array}{r}87.16 \\
103.85 \\
97.15\end{array}$ & $\begin{array}{l}174.09 \\
162.11 \\
167.06\end{array}$ & $\begin{array}{l}5.60 \\
5.60 \\
5.60\end{array}$ & $\begin{array}{l}1317.40 \\
1458.50 \\
1458.50\end{array}$ & $\begin{array}{l}222 \\
331 \\
553\end{array}$ \\
\hline $1.1-2.5$ & $\begin{array}{c}1 \\
2 \\
1 \& 2\end{array}$ & $\begin{array}{l}71.23 \\
72.02 \\
71.71\end{array}$ & $\begin{array}{l}77.50 \\
77.39 \\
77.43\end{array}$ & $\begin{array}{l}155.05 \\
106.63 \\
127.93\end{array}$ & $\begin{array}{l}5.60 \\
5.60 \\
5.60\end{array}$ & $\begin{array}{r}1396.40 \\
674.20 \\
1396.40\end{array}$ & $\begin{array}{l}117 \\
176 \\
293\end{array}$ \\
\hline $2.6-6.0$ & $\begin{array}{c}1 \\
2 \\
1 \& 2\end{array}$ & $\begin{array}{l}42.49 \\
52.09 \\
48.93\end{array}$ & $\begin{array}{l}43.63 \\
51.89 \\
49.09\end{array}$ & $\begin{array}{l}45.48 \\
65.92 \\
59.76\end{array}$ & $\begin{array}{l}5.60 \\
.5 .60 \\
5.60\end{array}$ & $\begin{array}{l}234.10 \\
301.80 \\
301.80\end{array}$ & $\begin{array}{r}56 \\
109 \\
165\end{array}$ \\
\hline $6.1-12.0$ & $\begin{array}{c}1 \\
2 \\
1 \& 2\end{array}$ & $\begin{array}{l}33.25 \\
28.45 \\
31.26\end{array}$ & $\begin{array}{l}47.97 \\
30.62 \\
37.35\end{array}$ & $\begin{array}{l}97.35 \\
38.07 \\
67.38\end{array}$ & $\begin{array}{l}5.60 \\
5.60 \\
5.60\end{array}$ & $\begin{array}{l}496.50 \\
222.90 \\
496.50\end{array}$ & $\begin{array}{l}26 \\
41 \\
67\end{array}$ \\
\hline$>12.0$ & $\begin{array}{c}1 \\
2 \\
1 \& 2\end{array}$ & $\begin{array}{l}1.6 .18 \\
14.84 \\
15.47\end{array}$ & $\begin{array}{l}16.36 \\
14.10 \\
15.29\end{array}$ & $\begin{array}{r}11.59 \\
7.47 \\
9.66\end{array}$ & $\begin{array}{l}5.60 \\
5.60 \\
5.60\end{array}$ & $\begin{array}{l}48.00 \\
25.40 \\
48.00\end{array}$ & $\begin{array}{r}10 \\
9 \\
19\end{array}$ \\
\hline
\end{tabular}


TABLE 12 (continued) Chloride.

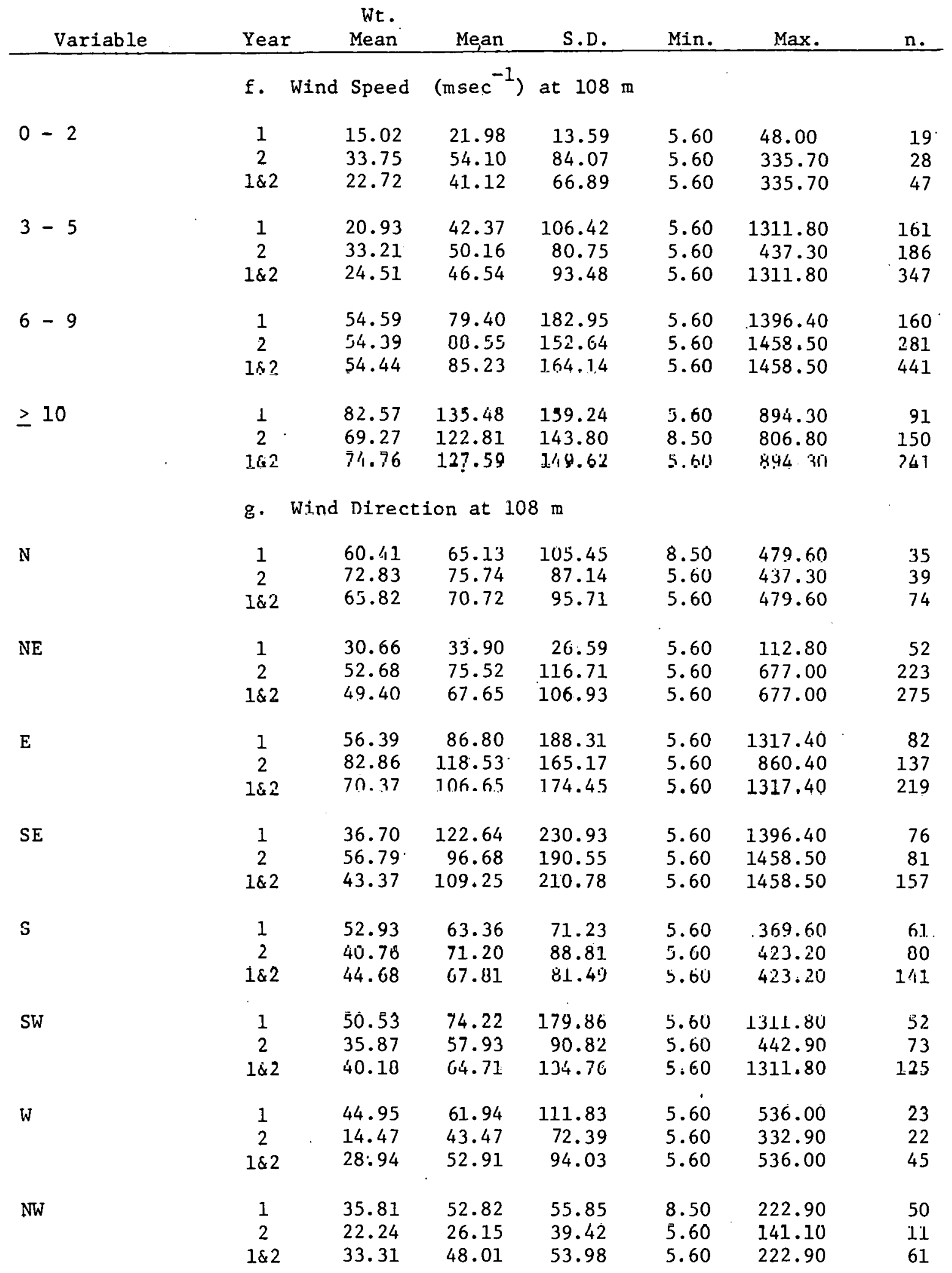


TABLE 12 (continued) Chloride

\begin{tabular}{|c|c|c|c|c|c|c|c|}
\hline Variable & Year & $\begin{array}{l}\text { Wt. } \\
\text { Mean }\end{array}$ & Męan & S.D. & Min. & Max. & n. \\
\hline & h. $\mathrm{T}$ & peratur & $\left({ }^{\circ} \mathrm{C}\right)$ & & & & \\
\hline-10.0 to -5.1 & $\begin{array}{c}1 \\
2 \\
1 \& 2\end{array}$ & $\begin{array}{l}22.60 \\
68.67 \\
40.13\end{array}$ & $\begin{array}{r}22.60 \\
134.73 \\
97.35\end{array}$ & $\begin{array}{r}0.00 \\
132.32 \\
117.72\end{array}$ & $\begin{array}{l}22.60 \\
62.10 \\
22.60\end{array}$ & $\begin{array}{r}22.60 \\
332.90 \\
332.90\end{array}$ & $\begin{array}{l}2 \\
4 \\
6\end{array}$ \\
\hline-5.0 to -0.1 & $\begin{array}{c}1 \\
2 \\
1 \& 2\end{array}$ & $\begin{array}{l}37.93 \\
61.29 \\
56.56\end{array}$ & $\begin{array}{l}40.78 \\
58.39 \\
53.84\end{array}$ & $\begin{array}{l}54.29 \\
70.03 \\
66.55\end{array}$ & $\begin{array}{l}8.50 \\
8.50 \\
8.50\end{array}$ & $\begin{array}{l}265.20 \\
355.40 \\
355.40\end{array}$ & $\begin{array}{r}31 \\
89 \\
120\end{array}$ \\
\hline 0.0 to 4.9 & $\begin{array}{c}1 \\
2 \\
1 \& 2\end{array}$ & $\begin{array}{l}61.32 \\
48.72 \\
53.76\end{array}$ & $\begin{array}{l}97.80 \\
58.57 \\
72.72\end{array}$ & $\begin{array}{l}175.49 \\
110.37 \\
138.27\end{array}$ & $\begin{array}{l}5.60 \\
5.60 \\
5.60\end{array}$ & $\begin{array}{r}1317.40 \\
674.20 \\
1317.40\end{array}$ & $\begin{array}{r}66 \\
117 \\
183\end{array}$ \\
\hline 5.0 to 9.9 & $\begin{array}{c}1 \\
2 \\
1 \& 2\end{array}$ & $\begin{array}{l}38.92 \\
77.80 \\
61.61\end{array}$ & $\begin{array}{r}65.39 \\
1.48 .82 \\
112.23\end{array}$ & $\begin{array}{l}157.11 \\
199.44 \\
186.41\end{array}$ & $\begin{array}{l}5.60 \\
8.50 \\
5.60\end{array}$ & $\begin{array}{l}1396.40 \\
1458.50 \\
1458.50\end{array}$ & $\begin{array}{l}107 \\
137 \\
244\end{array}$ \\
\hline 10.0 to 14.9 & $\begin{array}{c}1 \\
2 \\
1 \& 2\end{array}$ & $\begin{array}{l}64.93 \\
59.87 \\
61.23\end{array}$ & $\begin{array}{l}88.93 \\
83.49 \\
85.24\end{array}$ & $\begin{array}{l}155.87 \\
124.70 \\
135.20\end{array}$ & $\begin{array}{l}5.60 \\
5.60 \\
5.60\end{array}$ & $\begin{array}{r}1066.30 \\
860.40 \\
1066.30\end{array}$ & $\begin{array}{r}84 \\
178 \\
262\end{array}$ \\
\hline 15.0 to 19.9 & $\begin{array}{c}1 \\
2 \\
1 \& 2\end{array}$ & $\begin{array}{l}46.40 \\
40.83 \\
42.74\end{array}$ & $\begin{array}{l}57.77 \\
58.05 \\
57.94\end{array}$ & $\begin{array}{l}76.40 \\
78.17 \\
77.17\end{array}$ & $\begin{array}{r}14.10 \\
5.60 \\
5.60\end{array}$ & $\begin{array}{l}369.60 \\
426.00 \\
426.00\end{array}$ & $\begin{array}{r}46 \\
73 \\
119\end{array}$ \\
\hline 20.0 to 24.9 & $\begin{array}{c}1 \\
2 \\
1 \& 2\end{array}$ & $\begin{array}{l}36.88 \\
26.19 \\
33.74\end{array}$ & $\begin{array}{l}78.99 \\
44.34 \\
64.86\end{array}$ & $\begin{array}{r}176.65 \\
58.92 \\
141.76\end{array}$ & $\begin{array}{r}14.10 \\
5.60 \\
5.60\end{array}$ & $\begin{array}{r}1311.80 \\
369.60 \\
1311.80\end{array}$ & $\begin{array}{r}93 \\
64 \\
157\end{array}$ \\
\hline 25.0 to 29.9 & $\begin{array}{c}1 \\
2 \\
1 \& 2\end{array}$ & $\begin{array}{l}10.60 \\
14.28 \\
12.23\end{array}$ & $\begin{array}{l}18.35 \\
30.33 \\
26.33\end{array}$ & $\begin{array}{l}13.93 \\
22.53 \\
19.54\end{array}$ & $\begin{array}{l}8.50 \\
5.60 \\
5.60\end{array}$ & $\begin{array}{l}28.20 \\
50.80 \\
50.80\end{array}$ & $\begin{array}{l}2 \\
4 \\
6\end{array}$ \\
\hline
\end{tabular}




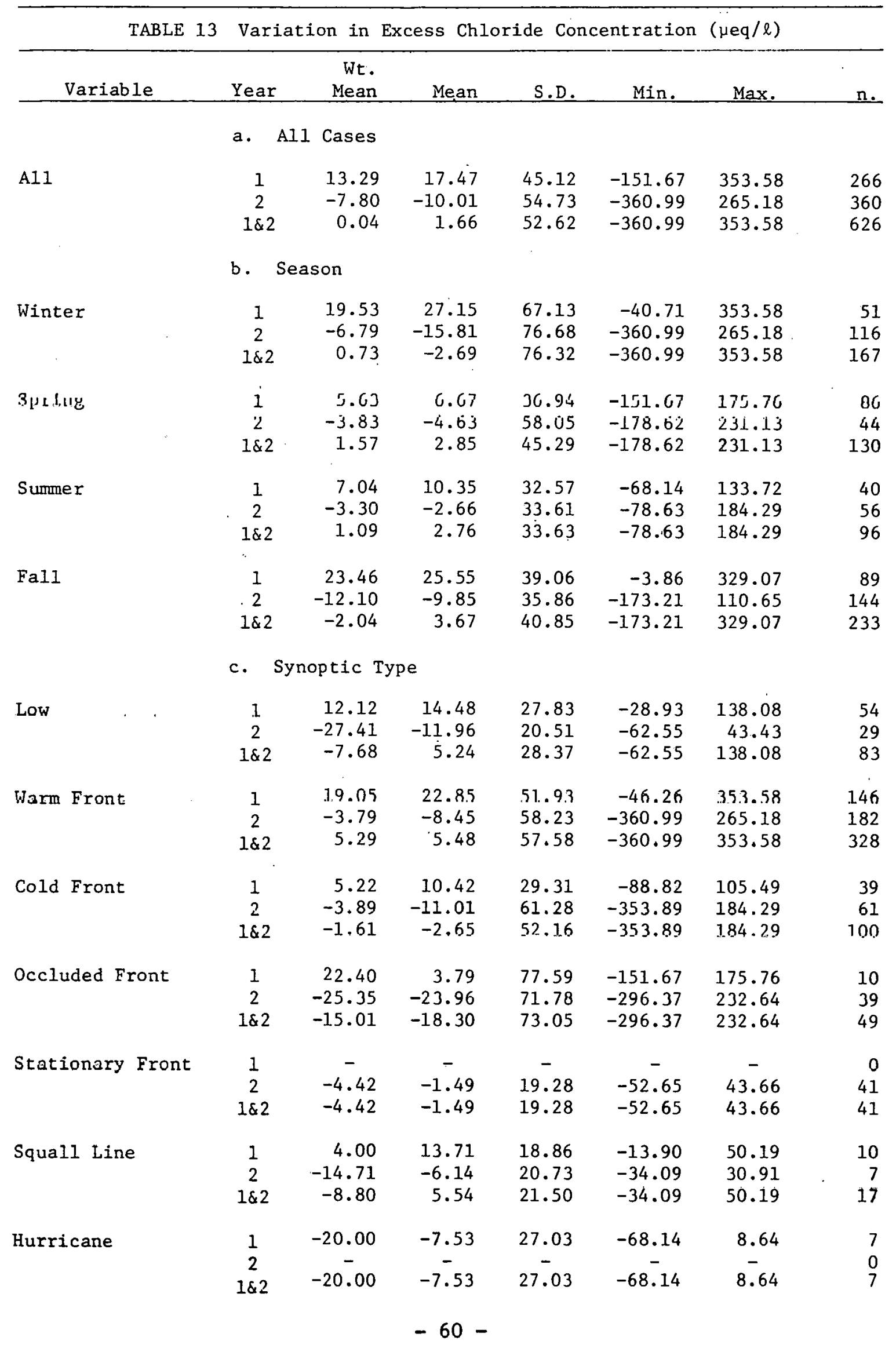


TABLE 13 (continued) Excess Chloride

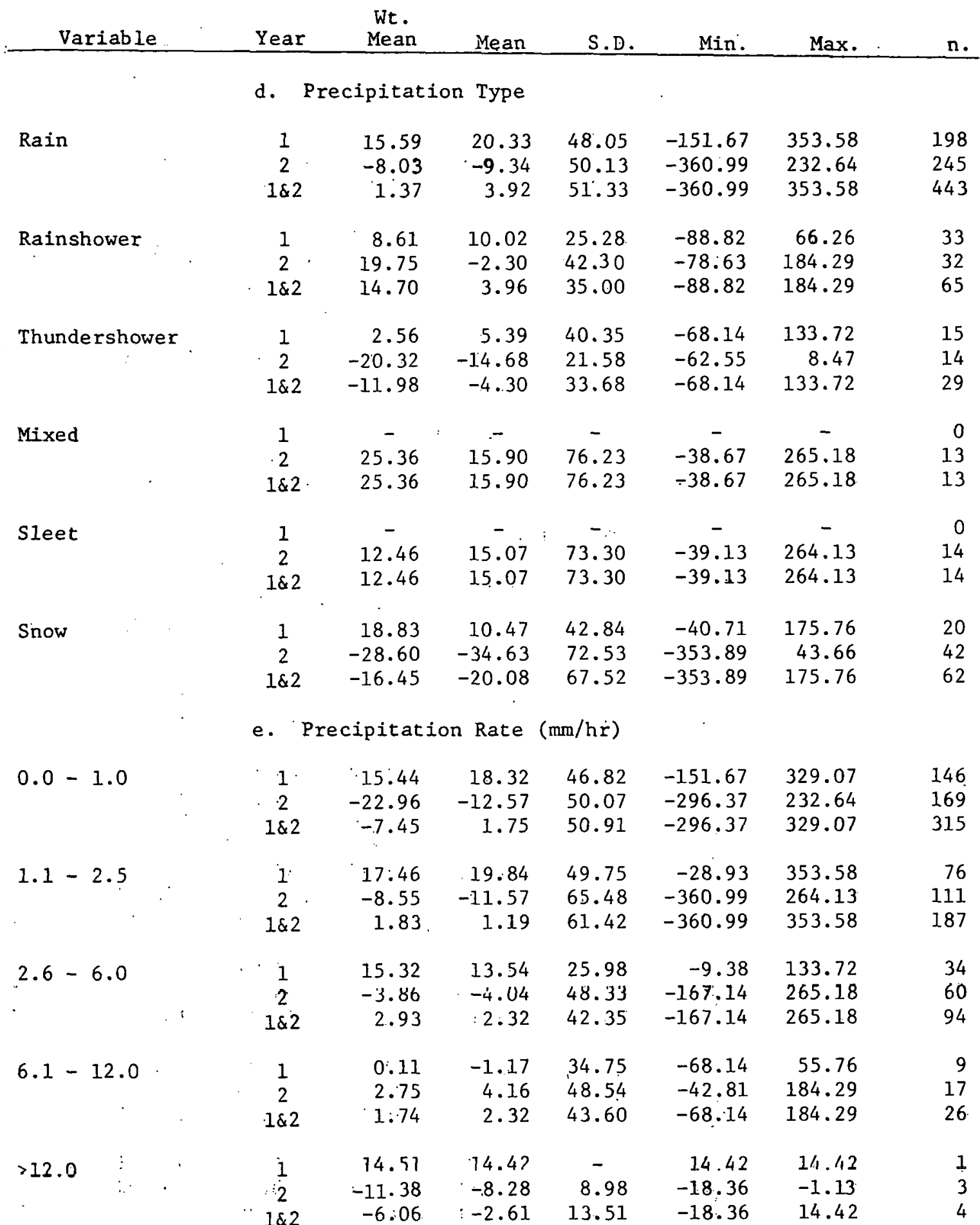


TABLE 13 (continued) Excess Chloride

\begin{tabular}{|c|c|c|c|c|c|c|c|}
\hline Variable & Year & $\begin{array}{l}\text { Wt. } \\
\text { Mean }\end{array}$ & Mean & S.D. & Min. & Max. & $\mathrm{n}$. \\
\hline & f. & Speed & $\left(\mathrm{msec}^{-}\right.$ & at 108 & a & & \\
\hline $0-2$ & $\begin{array}{c}1 \\
2 \\
1 \& 2\end{array}$ & $\begin{array}{l}7.90 \\
3.24 \\
6.54\end{array}$ & $\begin{array}{l}10.22 \\
12.95 \\
11.34\end{array}$ & $\begin{array}{l}14.82 \\
20.15 \\
16.66\end{array}$ & $\begin{array}{r}-5.69 \\
-12.97 \\
-12.97\end{array}$ & $\begin{array}{l}36.77 \\
43.66 \\
43.66\end{array}$ & $\begin{array}{r}10 \\
7 \\
17\end{array}$ \\
\hline $3-5$ & $\begin{array}{c}1 \\
2 \\
1 \& 2\end{array}$ & $\begin{array}{r}16.75 \\
-3.54 \\
8.43\end{array}$ & $\begin{array}{r}12.54 \\
1.91 \\
7.59\end{array}$ & $\begin{array}{l}27.32 \\
46.02 \\
37.46\end{array}$ & $\begin{array}{r}-88.82 \\
-173.21 \\
-173.21\end{array}$ & $\begin{array}{l}133.72 \\
232.64 \\
232.64\end{array}$ & $\begin{array}{r}78 \\
68 \\
146\end{array}$ \\
\hline $6-9$ & $\begin{array}{c}1 \\
2 \\
1 \& 2\end{array}$ & $\begin{array}{r}14.57 \\
-4.07 \\
1.33\end{array}$ & $\begin{array}{r}21.40 \\
-4.55 \\
5.81\end{array}$ & $\begin{array}{l}56.73 \\
52.46 \\
55.58\end{array}$ & $\begin{array}{r}-46.26 \\
-360.99 \\
-360.99\end{array}$ & $\begin{array}{l}353.58 \\
264.13 \\
353.58\end{array}$ & $\begin{array}{l}109 \\
164 \\
273\end{array}$ \\
\hline$\geq 10$ & $\begin{array}{c}1 \\
2 \\
1 \& 2\end{array}$ & $\begin{array}{r}10.74 \\
-17.98 \\
-6.28\end{array}$ & $\begin{array}{r}17.88 \\
-29.03 \\
-11.15\end{array}$ & $\begin{array}{l}43.46 \\
55.86 \\
56.21\end{array}$ & $\begin{array}{r}151.67 \\
-353.89 \\
-353.89\end{array}$ & $\begin{array}{r}175.76 \\
36.89 \\
175.76\end{array}$ & $\begin{array}{r}69 \\
112 \\
181\end{array}$ \\
\hline
\end{tabular}

g. Wind Direction at $108 \mathrm{~m}$

$\mathrm{N}$

$\begin{array}{crrrrrr}1 & 16.01 & 17.86 & 21.90 & -1.27 & 87.88 & 22 \\ 2 & -30.51 & -27.34 & 68.72 & -296.37 & 43.43 & 24 \\ 1 \& 2 & -5.38 & -5.72 & 56.20 & -296.37 & 87.88 & 46\end{array}$

$\mathrm{NE}$

$\begin{array}{lllllll}1 & 13.02 & 9.70 & 19.84 & -29.43 & 70.27 & 30\end{array}$

$\begin{array}{lllllll}2 & -6.54 & -9.84 & 53.69 & -150.41 & 265.18 & 113\end{array}$

$\begin{array}{lllllll}1 \& 2 & -3.42 & -5.74 & 49.17 & -150.41 & 265.18 & 1.43\end{array}$

E

$\begin{array}{rrrrrrr}1 & 6.07 & 12.42 & 35.38 & -68.14 & 138.08 & 46 \\ 2 & -0.68 & -2.62 & 49.35 & -195.61 & 231.13 & 93\end{array}$

$\begin{array}{lllllll}1 \& 2 & 1.68 & 2.36 & 45.63 & -195.61 & 231.13 & 139\end{array}$

$S E$

$\begin{array}{rrrrrrr}1 & 24.56 & 38.51 & 81.76 & -88.82 & 353.58 & 51 \\ 2 & -27.19 & -21.95 & 63.31 & -360.99 & 26.83 & 41 \\ 1 \& 2 & -2.39 & 11.56 & 79.67 & -360.99 & 353.58 & 92\end{array}$

S

$\begin{array}{lrrrrrr}1 & 11.92 & 12.95 & 21.39 & -32.88 & 64.42 & 44 \\ 2 & 5.52 & -1.15 & 40.74 & -178.62 & 184.29 & 47 \\ 18 & 8.19 & 5.67 & 33.43 & -178.62 & 184.29 & 91\end{array}$

$\begin{array}{llllllll}\text { SW } & 1 & 10.16 & 14.16 & 21.15 & \mathbf{- 2 3 . 8 9} & 70.09 & 35\end{array}$

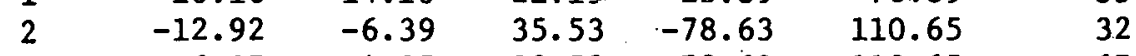

$\begin{array}{lllllll}1 \& 2 & -6.37 & 4.35 & 30.50 & -78.63 & 11.0 .65 & 67\end{array}$

W $\quad \begin{array}{lllllll}1 & 35.22 & 36.86 & 51.51 & 7.39 & 1 \% 3.76 & 10\end{array}$

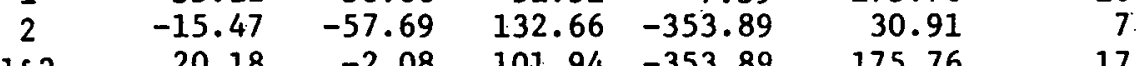

$\begin{array}{rrrrrrr}1 & 1.23 & -0.24 & 33.00 & -151.67 & 50.78 & 28 \\ 2 & -4.97 & -10.17 & 11 . / 0 & -22.70 & 0.46 & 3 \\ 1 \& 2 & -0.21 & -1.20 & 31.59 & -151.67 & 50.78 & 31\end{array}$ 
TABLF 13 (continued) Excess Chloride

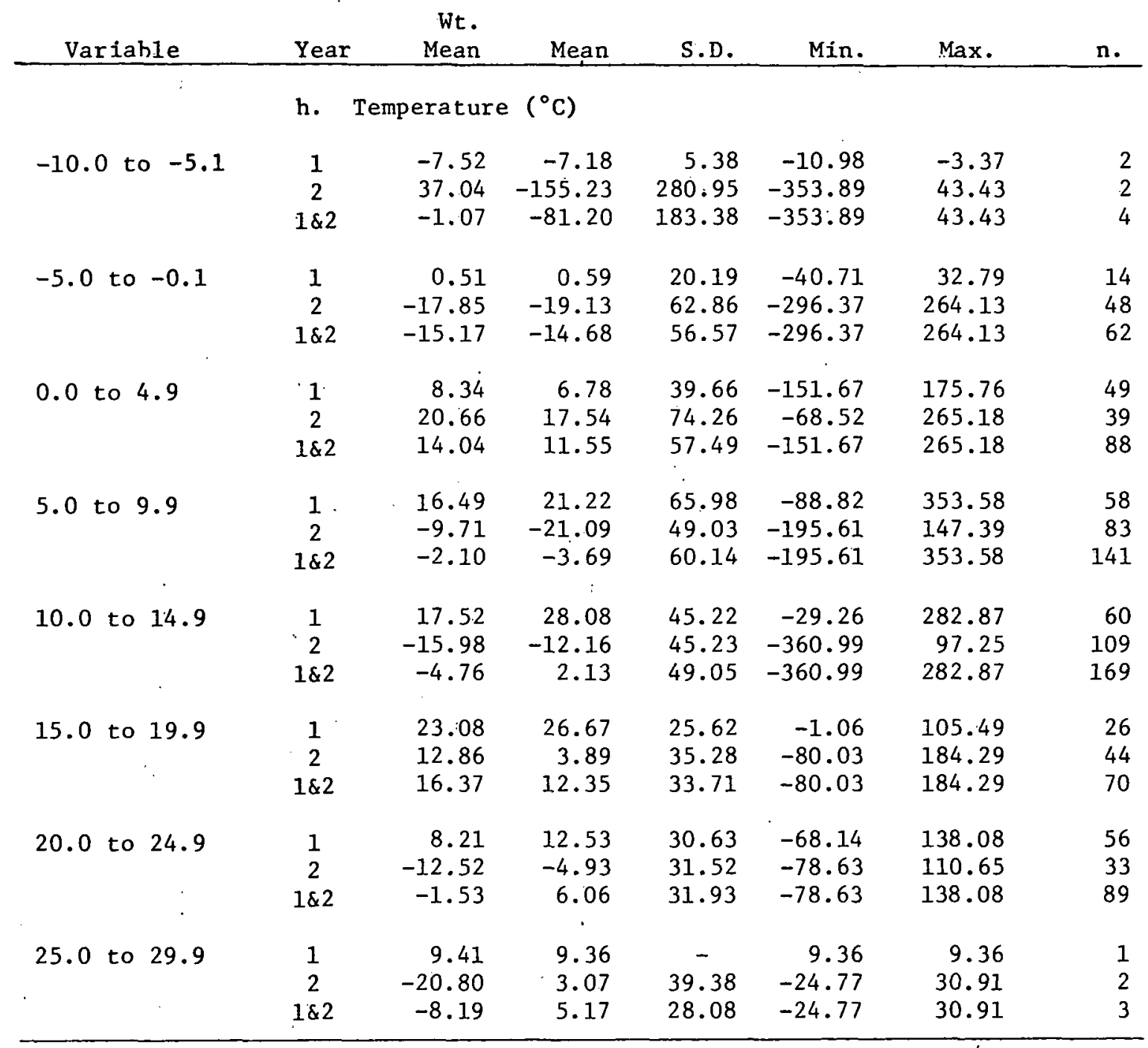




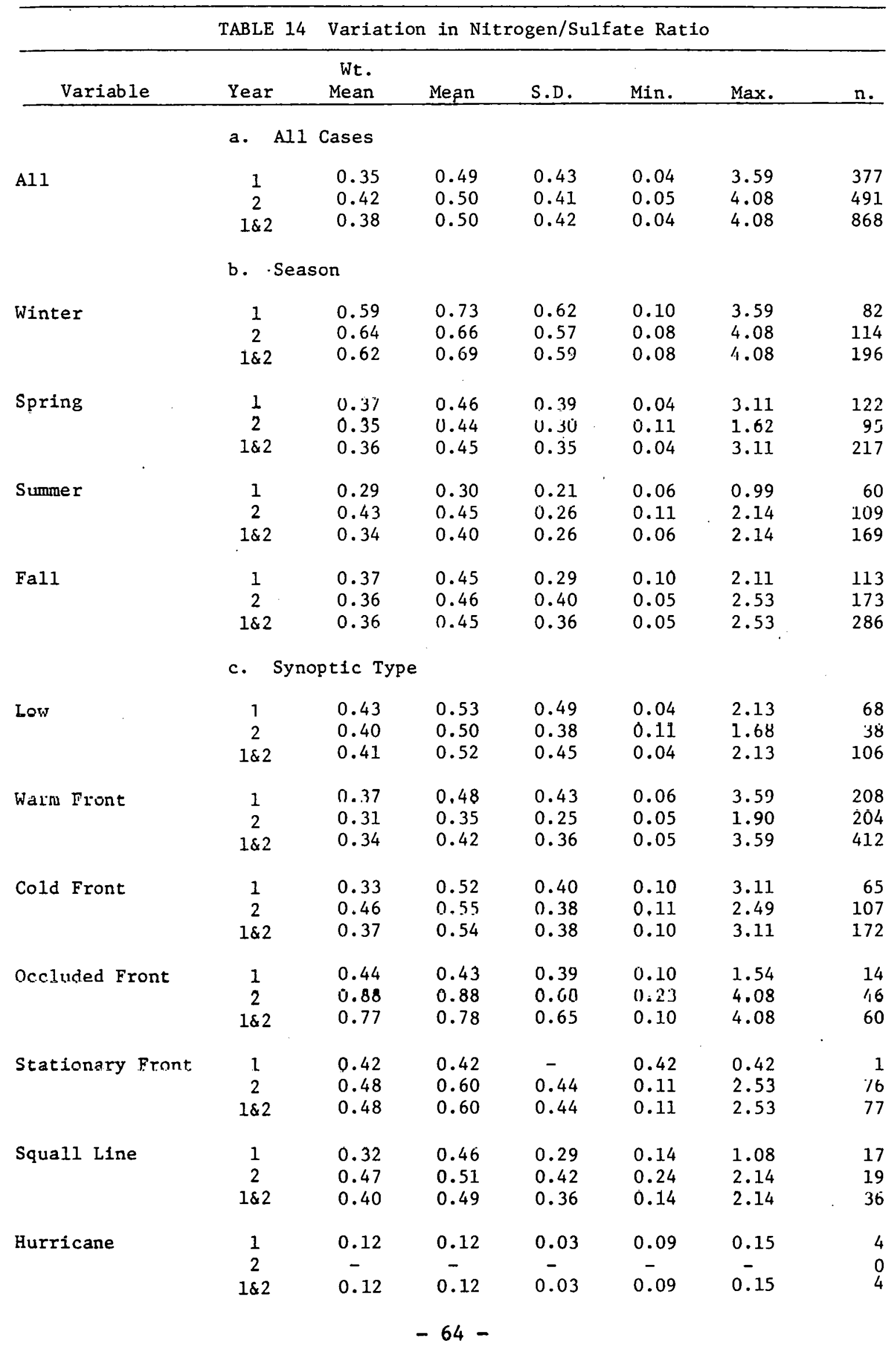


TABLE 14 (continued) Nitrogen/Sulfate

\begin{tabular}{|c|c|c|c|c|c|c|c|}
\hline Variable & Year & $\begin{array}{c}\text { Wt. } \\
\text { Mean }\end{array}$ & Męan & S.D. & Min. & Max. & $\mathrm{n}$. \\
\hline & d. $\quad \mathrm{P}$ & Litat & Type & & & & \\
\hline \multirow[t]{3}{*}{ Rain } & 1 & 0.31 & 0.39 & 0.27 & 0.06 & 2.11 & 262 \\
\hline & 2 & 0.33 & 0.41 & 0.34 & 0.05 & 2.53 & 321 \\
\hline & $1 \& 2$ & 0.32 & 0.40 & 0.31 & 0.05 & 2.53 & 583 \\
\hline \multirow[t]{3}{*}{ Rainshower } & 1 & 0.40 & 0.61 & 0.58 & 0.04 & 3.11 & 41 \\
\hline & 2 & 0.45 & 0.53 & 0.27 & 0.14 & 1.56 & 69 \\
\hline & $1 \& 2$ & 0.43 & 0.56 & 0.41 & 0.04 & 3.11 & 110 \\
\hline \multirow[t]{3}{*}{ Thundershower } & 1 & 0.32 & 0.46 & 0.24 & 0.10 & 1.11 & 25 \\
\hline & 2 & 0.44 & 0.49 & 0.37 & 0.24 & 2.14 & 27 \\
\hline & $1 \& 2$ & 0.37 & 0.48 & 0.31 & 0.10 & 2.14 & 52 \\
\hline \multirow[t]{3}{*}{ Mixed } & 1 & 0.66 & 0.66 & 0.19 & 0.48 & 0.90 & 4 \\
\hline & 2 & 0.48 & 0.50 & 0.16 & 0.29 & 0.73 & 8 \\
\hline & $1 \& 2$ & 0.60 & 0.56 & 0.18 & 0.29 & 0.90 & 12 \\
\hline \multirow[t]{3}{*}{ Sleet } & 1 & 0.39 & 0.39 & - & 0.39 & 0.39 & 1 \\
\hline & 2 & 0.46 & 0.55 & 0.15 & 0.33 & 0.76 & 7 \\
\hline & $1 \& 2$ & 0.45 & 0.53 & 0.15 & 0.33 & 0.76 & 8 \\
\hline \multirow[t]{4}{*}{ Snow } & 1 & 0.82 & 0.98 & 0.70 & 0.12 & 3.59 & 44 \\
\hline & 2 & 0.99 & 0.98 & 0.62 & 0.11 & 4.08 & 59 \\
\hline & $1 \& 2$ & 0.91 & 0.98 & 0.65 & 0.11 & 4.08 & 103 \\
\hline & e. $P$ & pitati & Rate ( & $/ \mathrm{hr})$ & & & . \\
\hline \multirow[t]{3}{*}{$0.0-1.0$} & 1 & 0.56 & 0.58 & 0.50 & 0.04 & 3.59 & 208 \\
\hline & 2 & 0.53 & 0.56 & 0.42 & 0.05 & 2.53 & 288 \\
\hline & $1 \& 2$ & 0.55 & 0.57 & 0.46 & 0.04 & 3.59 & 496 \\
\hline \multirow[t]{3}{*}{$1.1-2.5$} & 1 & 0.42 & 0.43 & 0.30 & 0.09 & 1.70 & 109 \\
\hline & 2 & 0.44 & 0.46 & 0.43 & 0.07 & 4.08 & 123 \\
\hline & $1 \& 2$ & 0.43 & 0.45 & 0.38 & 0.07 & 4.08 & 232 \\
\hline \multirow[t]{3}{*}{$2.6-6.0$} & 1 & 0.26 & 0.27 & 0.15 & 0.06 & $n .7 n$ & 43 \\
\hline & 2 & 0.34 & 0.33 & 0.21 & 0.12 & 1.33 & 59 \\
\hline & $1 \& 2$ & 0.31 & 0.30 & 0.19 & 0.06 & 1.33 & 102 \\
\hline \multirow[t]{3}{*}{$6.1-12.0$} & 1 & 0.29 & 0.29 & 0.13 & 0.12 & 0.64 & 12 \\
\hline & 2 & 0.48 & 0.45 & 0.52 & 0.14 & 2.14 & 15 \\
\hline & $1 \& 2$ & 0.35 & 0.38 & 0.40 & 0.12 & 2.14 & 27 \\
\hline \multirow[t]{3}{*}{$= \pm 2.0$} & 1 & 0.30 & 0.34 & 0.26 & 0.14 & 0.77 & 3 \\
\hline & 2 & 0.28 & 0.29 & 0.05 & 0.24 & 0.36 & 6 \\
\hline & $1 \& 2$ & 0.29 & 0.31 & 0.17 & 0.14 & 0.77 & 11 \\
\hline
\end{tabular}


TABLE 14 (continued) Nitrogen/Sulfate

\begin{tabular}{|c|c|c|c|c|c|c|c|}
\hline Variable & Year & $\begin{array}{l}\text { Wt. } \\
\text { Mean }\end{array}$ & Mean & S.D. & Min. & Max. & n. \\
\hline & f. $h$ & Speed & $m \sec ^{-1}$ & 108 & & & \\
\hline $0-2$ & 1 & 0.48 & 0.78 & 0.56 & 0.10 & 2.11 & 18 \\
\hline & 2 & 0.83 & 0.79 & 0.38 & 0.20 & 1.45 & 21 \\
\hline & $1 \& 2$ & 0.62 & 0.78 & 0.47 & 0.10 & 2.11 & 39 \\
\hline $3-5$ & 1 & 0.34 & 0.50 & 0.38 & 0.04 & 1.90 & 145 \\
\hline & 2 & 0.46 & 0.59 & 0.50 & 0.11 & 4.08 & 157 \\
\hline & $1 \& 2$ & 0.37 & 0.55 & 0.45 & 0.04 & 4.08 & 302 \\
\hline $6-9$ & 1 & 0.40 & 0.50 & 0.45 & 0.09 & 3.59 & 154 \\
\hline & 2 & 0.40 & 0.46 & 0.36 & 0.05 & 2.53 & 222 \\
\hline & $1 \leqslant 2$ & 0.40 & 0.47 & 0.40 & 0.05 & 3.59 & 376 \\
\hline$\geq 10$ & $\begin{array}{l}1 \\
2\end{array}$ & $\begin{array}{l}0.28 \\
0.35\end{array}$ & $\begin{array}{l}0.35 \\
0.39\end{array}$ & $\begin{array}{l}0.36 \\
0.32\end{array}$ & $\begin{array}{l}0.10 \\
0.07\end{array}$ & $\begin{array}{l}2.13 \\
1.90\end{array}$ & $\begin{array}{l}.60 \\
89\end{array}$ \\
\hline & 182 & $1.3 \%$ & 0.38 & 0.34 & 0.07 & 2.1 .3 & 14.9 \\
\hline & g. W & Direct & $n$ at 1 & m & & & \\
\hline $\mathrm{N}$ & 1 & 0.47 & 0.61 & 0.44 & 0.14 & 1.90 & 33 \\
\hline & 2 & 0.59 & 0.68 & 0.46 & 0.19 & 2.24 & 39 \\
\hline & $1 \& 2$ & 0.52 & 0.64 & 0.45 & 0.14 & 2.24 & 72 \\
\hline $\mathrm{NE}$ & 1 & 0.38 & 0.58 & 0.56 & 0.04 & 2.48 & 52 \\
\hline & 2 & 0.47 & 0.54 & 0.43 & 0.11 & 2.49 & 146 \\
\hline & $1 \& 2$ & 0,45 & 0.55 & 0.47 & 0.04 & 2.49 & 198 \\
\hline$E$ & 1 & 0.31 & 0.41 & 0.48 & 0.10 & 3.59 & 59 \\
\hline & 2 & 0.31 & 0.40 & 0.46 & 0.05 & 4.08 & 105 \\
\hline & $1 \& 2$ & 0.31 & 0.40 & 0.47 & n. $n 5$ & $4 . n 8$ & 164 \\
\hline SE & 1 & 0.30 & 0.38 & 0.29 & 0.10 & 1.60 & 61 \\
\hline & 2 & 0.36 & 0.49 & 0.42 & 0.08 & 2.53 & 59 \\
\hline . & $1 \& 2$ & 0.31 & 0.44 & 0.37 & 0.08 & 2.53 & 120 \\
\hline S & 1 & 0.32 & 0.41 & 0.38 & 0.06 & 2.11 & 5.5 \\
\hline & 2 & 0.33 & 0.39 & 0.1 .9 & 0.11 & 0.98 & 51 \\
\hline & $1 \& 2$ & 0.33 & 0.40 & 0.30 & 0.06 & 2.11 & 106 \\
\hline SW & 1 & 0.54 & 0.62 & 0.44 & 0.24 & 3.11 & 48 \\
\hline & 2 & 0.57 & 0.57 & 0.39 & 0.12 & 2.14 & 57 \\
\hline & $1 \& 2$ & 0.53 & 0.60 & 0.41 & 0.12 & 3.11 & -105 \\
\hline W & 1 & 0.57 & 0.52 & 0.18 & 0.27 & 0.86 & $2-1$ \\
\hline & 2 & 0.45 & $0 .-52$ & 0.25 & 0.11 & 1.19 & 22 \\
\hline & $1 \& 2$ & 0.51 & 0.52 & 0.22 & 0.11 & 1.19 & 43 \\
\hline NW & 1 & 0.39 & 0.49 & 0.38 & 0.12 & 2.13 & 48 \\
\hline & 2 & 0.48 & 0.54 & 0.30 & 0.16 & 1.30 & 12. \\
\hline & $1 \& 2$ & $0: 40$ & $0.50=$ & 0.36 & 0.12 & .2 .13 & 60. \\
\hline
\end{tabular}


TABLE 14 (continued) Nitrogen/Sulfate

\begin{tabular}{|c|c|c|c|c|c|c|c|}
\hline Variable & Year & $\begin{array}{l}\text { Wt. } \\
\text { Mean }\end{array}$ & Mean & S.D. & Min. & Max. & n. \\
\hline & \multicolumn{7}{|c|}{ Temperature $\left({ }^{\circ} \mathrm{C}\right)$} \\
\hline-10.0 to -5.1 & $\begin{array}{c}1 \\
2 \\
1 \& 2\end{array}$ & $\begin{array}{l}1.67 \\
0.32 \\
1.47\end{array}$ & $\begin{array}{l}1.71 \\
0.22 \\
0.97\end{array}$ & $\begin{array}{l}0.58 \\
0.15 \\
0.93\end{array}$ & $\begin{array}{l}1.30 \\
0.11 \\
0.11\end{array}$ & $\begin{array}{l}2.13 \\
0.32 \\
2.13\end{array}$ & $\begin{array}{r}.2 \\
2 \\
4\end{array}$ \\
\hline-5.0 to -0.1 & $\begin{array}{c}1 \\
2 \\
1 \& 2\end{array}$ & $\begin{array}{l}0.89 \\
1.01 \\
0.96\end{array}$ & $\begin{array}{l}0.99 \\
1.03 \\
1.02\end{array}$ & $\begin{array}{l}0.55 \\
0.66 \\
0.61\end{array}$ & $\begin{array}{l}0.23 \\
0.40 \\
0.23\end{array}$ & $\begin{array}{l}2.48 \\
4.08 \\
4.08\end{array}$ & $\begin{array}{l}33 \\
45 \\
78\end{array}$ \\
\hline 0.0 to 4.9 & $\begin{array}{c}1 \\
2 \\
1 \& 2\end{array}$ & $\begin{array}{l}0.39 \\
0.63 \\
0.50\end{array}$ & $\begin{array}{l}0.52 \\
0.62 \\
0.58\end{array}$ & $\begin{array}{l}0.55 \\
0.42 \\
0.48\end{array}$ & $\begin{array}{l}0.12 \\
0.11 \\
0.11\end{array}$ & $\begin{array}{l}3.59 \\
2.49 \\
3.59\end{array}$ & $\begin{array}{r}62 \\
84 \\
146\end{array}$ \\
\hline 5.0 to 9.9 & $\begin{array}{c}1 \\
2 \\
1 \& 2\end{array}$ & $\begin{array}{l}0.40 \\
0.29 \\
0.34\end{array}$ & $\begin{array}{l}0.50 \\
0.37 \\
0.43\end{array}$ & $\begin{array}{l}0.30 \\
0.28 \\
0.30\end{array}$ & $\begin{array}{l}0.10 \\
0.08 \\
0.08\end{array}$ & $\begin{array}{l}2.11 \\
1.62 \\
2.11\end{array}$ & $\begin{array}{r}93 \\
95 \\
188\end{array}$ \\
\hline 10.0 to 14.9 & $\begin{array}{c}1 \\
2 \\
1 \& 2\end{array}$ & $\begin{array}{l}0.34 \\
0.31 \\
0.32\end{array}$ & $\begin{array}{l}0.43 \\
0.37 \\
0.39\end{array}$ & $\begin{array}{l}0.40 \\
0.26 \\
0.32\end{array}$ & $\begin{array}{l}0.04 \\
0.05 \\
0.04\end{array}$ & $\begin{array}{l}3.11 \\
2.03 \\
3.11\end{array}$ & $\begin{array}{r}75 \\
135 \\
210\end{array}$ \\
\hline 15.0 to 19.9 & $\begin{array}{c}1 \\
2 \\
1 \& 2\end{array}$ & $\begin{array}{l}0.44 \\
0.34 \\
0.37\end{array}$ & $\begin{array}{l}0.41 \\
0.45 \\
0.44\end{array}$ & $\begin{array}{l}0.22 \\
0.35 \\
0.31\end{array}$ & $\begin{array}{l}0.10 \\
0.11 \\
0.10\end{array}$ & $\begin{array}{l}1.08 \\
2.53 \\
2.53\end{array}$ & $\begin{array}{r}35 \\
68 \\
103\end{array}$ \\
\hline 20.0 to 24.9 & $\begin{array}{c}1 \\
2 \\
1 \& 2\end{array}$ & $\begin{array}{l}0.27 \\
0.45 \\
0.33\end{array}$ & $\begin{array}{l}0.31 \\
0.50 \\
0.39\end{array}$ & $\begin{array}{l}0.21 \\
0.34 \\
0.29\end{array}$ & $\begin{array}{l}0.06 \\
0.12 \\
0.06\end{array}$ & $\begin{array}{l}0.99 \\
2.14 \\
2.14\end{array}$ & $\begin{array}{r}75 \\
58 \\
133\end{array}$ \\
\hline 25.0 to 29.9 & $\begin{array}{c}1 \\
2 \\
1 \& 2\end{array}$ & $\begin{array}{l}0.62 \\
0.57 \\
0.60\end{array}$ & $\begin{array}{l}0.55 \\
0.73 \\
0.67\end{array}$ & $\begin{array}{l}0.13 \\
0.23 \\
0.21\end{array}$ & $\begin{array}{l}0.45 \\
0.47 \\
0.45\end{array}$ & $\begin{array}{l}0.64 \\
0.98 \\
0.98\end{array}$ & $\begin{array}{l}2 \\
4 \\
6\end{array}$ \\
\hline
\end{tabular}




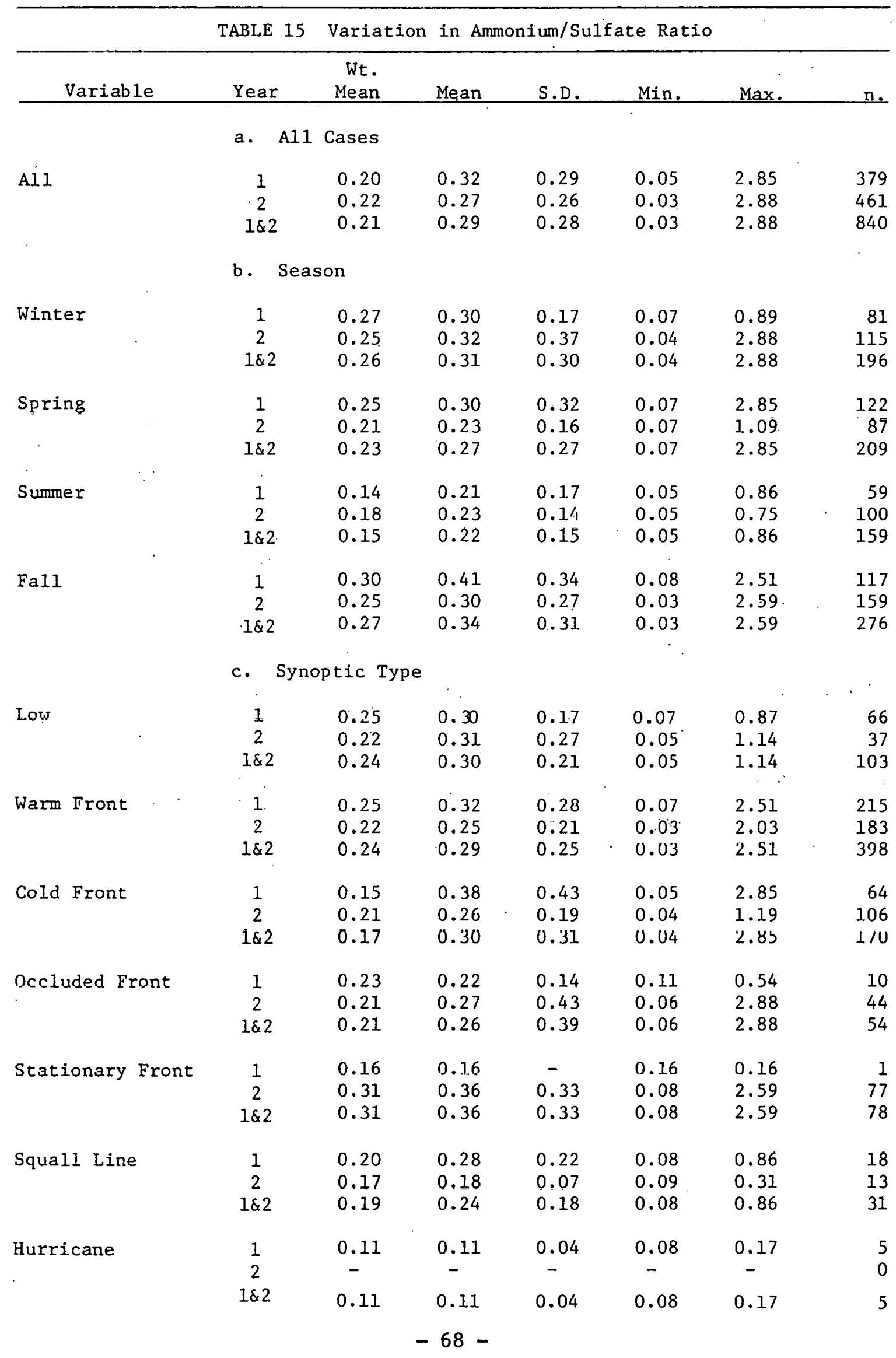


TABLE 15 (continued) Ammonium/Sulfate

\begin{tabular}{|c|c|c|c|c|c|c|c|}
\hline Variable & Year & $\begin{array}{l}\text { Wt. } \\
\text { Mean }\end{array}$ & Mean & S.D. & Min. & Max. & n. \\
\hline & d. $P$ & ipitat & Type & & & & \\
\hline \multirow[t]{3}{*}{ Rain } & 1 & 0.24 & 0.31 & 0.26 & 0.05 & 2.51 & 264 \\
\hline & 2 & 0.23 & 0.28 & 0.30 & 0.03 & 2.88 & 296 \\
\hline & $1 \& 2$ & 0.23 & 0.29 & 0.28 & 0.03 & 2.88 & 560 \\
\hline \multirow[t]{3}{*}{ Rainshower } & 1 & 0.26 & 0.46 & 0.51 & 0.08 & 2.85 & 42 \\
\hline & 2 & 0.23 & 0.26 & 0.15 & 0.07 & 0.74 & 68 \\
\hline & $1 \& 2$ & 0.24 & 0.34 & 0.35 & 0.07 & 2.85 & 110 \\
\hline \multirow[t]{3}{*}{ Thundershower } & 1 & 0.14 & 0.22 & 0.17 & 0.10 & 0.89 & 25 \\
\hline & 2 & 0.20 & 0.21 & 0.09 & 0.09 & 0.41 & 24 \\
\hline & $1 \& 2$ & 0.16 & 0.22 & 0.13 & 0.09 & 0.89 & 49 \\
\hline \multirow[t]{3}{*}{ Mixed } & 1. & 0.28 & 0.28 & 0.03 & 0.25 & 0.33 & 5 \\
\hline & 2 & 0.26 & 0.25 & 0.10 & 0.12 & 0.36 & 8 \\
\hline & $1 \& 2$ & 0.28 & 0.26 & 0.08 & 0.12 & 0.36 & 13 \\
\hline \multirow[t]{3}{*}{ Sleet } & 1 & 0.19 & 0.19 & - & 0.19 & 0.19 & 1 \\
\hline & 2 & 0.18 & 0.32 & 0.34 & 0.12 & 0.98 & 6 \\
\hline & $1 \& 2$ & 0.18 & 0.30 & 0.31 & 0.12 & 0.98 & 7 \\
\hline \multirow[t]{3}{*}{ Snow } & 1 & 0.28 & 0.31 & 0.17 & 0.08 & 0.75 & 42 \\
\hline & 2 & 0.27 & 0.28 & 0.21 & 0.06 & 1.14 & 59 \\
\hline & $1 \& 2$ & 0.27 & 0.29 & 0.20 & 0.06 & 1.14 & 101 \\
\hline & e. $P$ & pitat & Rate & / $/ \mathrm{hr})$ & & & \\
\hline \multirow[t]{3}{*}{$0.0-1.0$} & 1 & 0.33 & 0.39 & 0.35 & 0.05 & 2.85 & 215 \\
\hline & 2 & 0.26 & 0.31 & 0.31 & 0.03 & 2.88 & 277 \\
\hline & $1 \& 2$ & 0.29 & 0.34 & 0.33 & 0.03 & 2.88 & 492 \\
\hline \multirow[t]{3}{*}{$1.1-2.5$} & 1 & 0.25 & 0.25 & 0.17 & 0.08 & 1.48 & 104 \\
\hline & 2 & 0.22 & 0.22 & 0.15 & 0.06 & 1.06 & 112 \\
\hline & $1 \& 2$ & 0.23 & 0.24 & 0.16 & 0.06 & 1.48 & 216 \\
\hline \multirow[t]{3}{*}{$2.6-6.0$} & 1 & 0.19 & 0.20 & 0.11 & 0.07 & 0.47 & 41 \\
\hline & 2 . & 0.20 & 0.20 & 0.15 & 0.04 & 0.94 & 53 \\
\hline & $1 \& 2$ & 0.20 & 0.20 & 0.13 & 0.04 & 0.94 & 94 \\
\hline \multirow[t]{3}{*}{$6.1-12.0$} & 1 & 0.14 & 0.21 & 0.10 & 0.10 & 0.46 & 12 \\
\hline & 2 & 0.22 & 0.23 & 0.11 & 0.09 & 0.49 & 13 \\
\hline & $1 \& 2$ & 0.16 & 0.22 & 0.10 & 0.09 & 0.49 & 25 \\
\hline \multirow[t]{3}{*}{$>12.0$} & 1 & U.1I & U.16 & 0.09 & 0.06 & $0 . j u$ & 7 \\
\hline & 2 & 0.22 & 0.24 & 0.07 & 0.13 & 0.30 & 6 \\
\hline & $1 \& 2$ & 0.20 & 0.20 & 0.09 & 0.06 & 0.30 & 13 \\
\hline
\end{tabular}


TABLE 15 (continued) Ammonium/Sulfate

Wt .

\begin{tabular}{|c|c|c|c|c|c|c|c|}
\hline Variable & Year & Mean & Mean & S.D. & Min. & Max. & $\mathbf{n}$ \\
\hline & f. & Speed & (msec $^{-1}$ & at 108 & $\mathrm{~m}$ & & \\
\hline $0-2$ & $\begin{array}{c}1 \\
2 \\
1 \& 2\end{array}$ & $\begin{array}{l}0.19 \\
0.30 \\
0.23\end{array}$ & $\begin{array}{l}0.31 \\
0.38 \\
0.34\end{array}$ & $\begin{array}{l}0.19 \\
0.20 \\
0.20\end{array}$ & $\begin{array}{r}0.08 \\
0.07 \\
0.07\end{array}$ & $\begin{array}{l}0.78 \\
0.98 \\
0.98\end{array}$ & $\begin{array}{l}20 \\
19 \\
39\end{array}$ \\
\hline $3-5$ & $\begin{array}{c}1 \\
2 \\
1 \& 2\end{array}$ & $\begin{array}{l}0.18 \\
0.27 \\
0.20\end{array}$ & $\begin{array}{l}0.33 \\
0.33 \\
0.33\end{array}$ & $\begin{array}{l}0.30 \\
0.37 \\
0.34\end{array}$ & $\begin{array}{l}0.06 \\
0.07 \\
0.06\end{array}$ & $\begin{array}{l}2.51 \\
2.88 \\
2.88\end{array}$ & $\begin{array}{l}140 \\
151 \\
291\end{array}$ \\
\hline $6-9$ & $\begin{array}{c}1 . \\
2 \\
1.2\end{array}$ & $\begin{array}{l}0.27 \\
0.21 \\
0.23\end{array}$ & $\begin{array}{l}0.33 \\
0.26 \\
0.21\end{array}$ & $\begin{array}{l}0.31 \\
0.20 \\
0.25\end{array}$ & $\begin{array}{l}0.05 \\
0.03 \\
0.03\end{array}$ & $\begin{array}{l}2.85 \\
1.09 \\
2.85\end{array}$ & $\begin{array}{l}155 \\
206 \\
361\end{array}$ \\
\hline$\geq 10$ & $\begin{array}{c}1 \\
2 \\
1 \& 2\end{array}$ & $\begin{array}{l}0.20 \\
0.19 \\
0.20\end{array}$ & $\begin{array}{l}0.26 \\
0.20 \\
0.23\end{array}$ & $\begin{array}{l}0.23 \\
0.14 \\
0.19\end{array}$ & $\begin{array}{l}0.07 \\
0.03 \\
0.03\end{array}$ & $\begin{array}{l}1.48 \\
0.85 \\
1.48\end{array}$ & $\begin{array}{r}64 \\
83 \\
147\end{array}$ \\
\hline
\end{tabular}

g. Wind Direction at $108 \mathrm{~m}$.

N

$\begin{array}{ccccccc}1 & 0.28 & 0.34 & 0.15 & 0.07 & 0.70 & 33 \\ 2 & 0.18 & 0.25 & 0.25 & 0.05 & 1.14 & 37 \\ 1 \& 2 & 0.24 & 0.29 & 0.21 & 0.05 & 1.14 & 70\end{array}$

NE

E

$\begin{array}{ccccccr}1 & 0.22 & 0.31 & 0.20 & 0.08 & 0.87 & 50 \\ 2 & 0.27 & 0.32 & 0.35 & 0.05 & 2.88 & 137 \\ 1 \& 2 & 0.26 & 0.32 & 0.32 & 0.05 & 2.88 & 187\end{array}$

$\begin{array}{cccccrrr}1 & 0.23 & 0.28 & 0.15 & 0.07 & 0.75 & 61 \\ 2 & 0.25 & 0.29 & 0.27 & 0.03 & 2.03 & 97 \\ 1 \& 2 & 11.24 & 0.24 & 0.23 & 0.03 & 2.03 & 158\end{array}$

$\begin{array}{cccccccr}\mathrm{SE} & 1 & 0.15 & 0.26 & 0.18 & 0.06 & 1.10 & 63 \\ 2 & 0.18 & 0.20 & 0.12 & 0.04 & 0.85 & 57 \\ & 1 \& 2 & 0.15 & 0.23 & 0.16 & 0.04 & 1.10 & 120\end{array}$

$\begin{array}{ccccccrr}\mathrm{S} & 1 & 0.27 & 0.43 & 0.44 & 0.05 & 2.51 & 59 \\ 2 & 0.19 & 0.22 & 0.11 & 0.06 & 0.53 & 52 \\ 1 \& 2 & 0.23 & 0.33 & 0.34 & 0.05 & 2.51 & 111\end{array}$

$\begin{array}{cccccccc}\text { SW } & 1 & 0.27 & 0.39 & 0.42 & 0.12 & 2.85 & 48 \\ 2 & 0.17 & 0.27 & 0.20 & 0.08 & 1.05 & 49 \\ 1 \& 2 & 0.21 & 0.33 & 0.33 & 0.08 & 2.85 & 97\end{array}$

$\begin{array}{cccccccc}W & 1 & 0.25 & 0.34 & 0.42 & 0.12 & 2.15 & 21 \\ 2 & 0.26 & 0.30 & 0.25 & 0.08 & 1.19 & 21 \\ 1 \& 2 & 0.26 & 0.32 & 0.34 & 0.08 & 2.15 & 42\end{array}$

$\begin{array}{cccccccc}\text { NW } & 1 & 0.22 & 0.22 & 0.10 & 0.08 & 0.50 & 1 / 1 \\ 2 & 0.28 & 0.22 & 0.12 & 0.08 & 0.41 & 11 \\ 1 \& 2 & 0.23 & 0.22 & 0.11 & 0.08 & 0.50 & 55\end{array}$


TABLE 15 (continued) Ammonium/Sulfate

Wt.

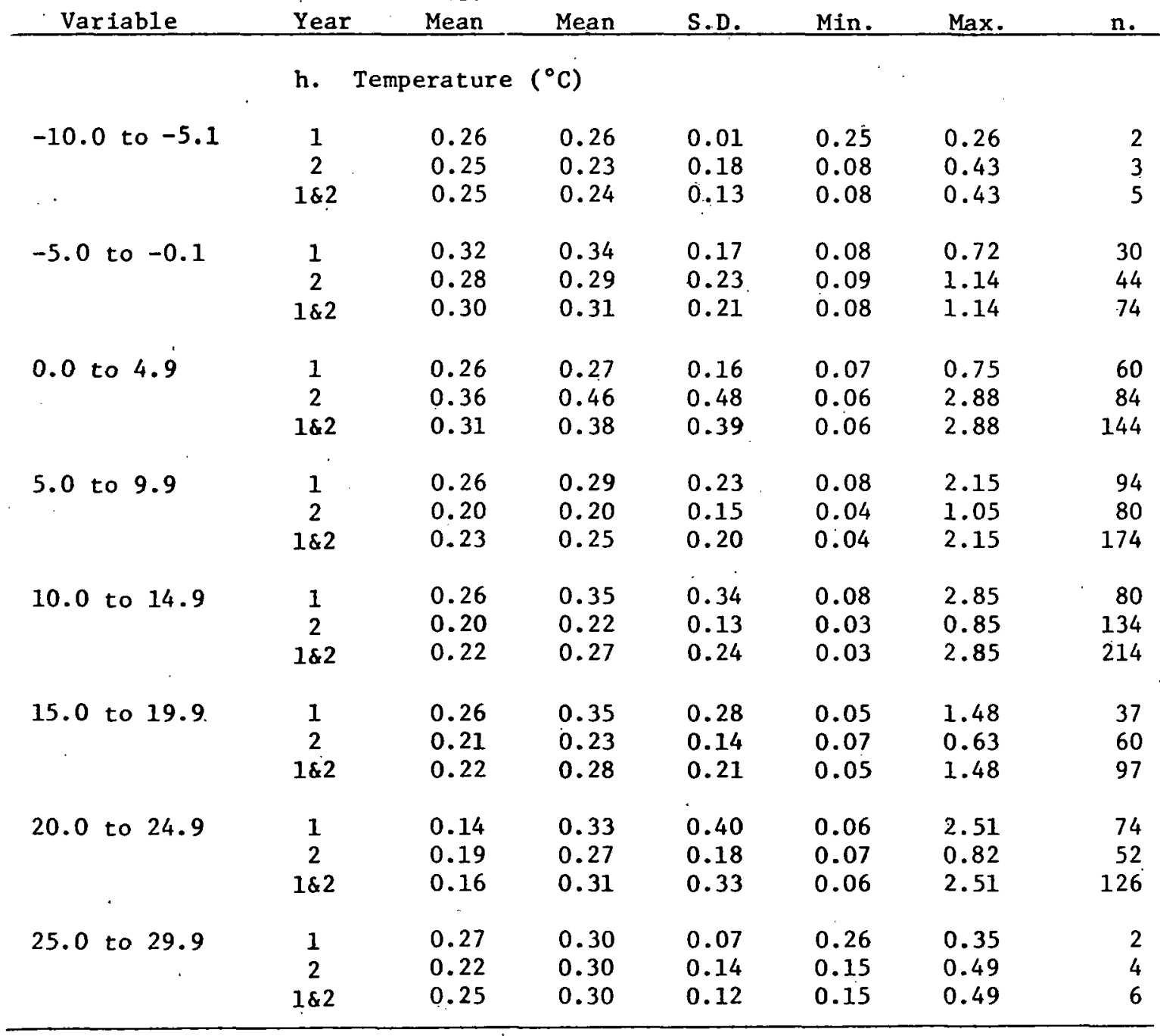




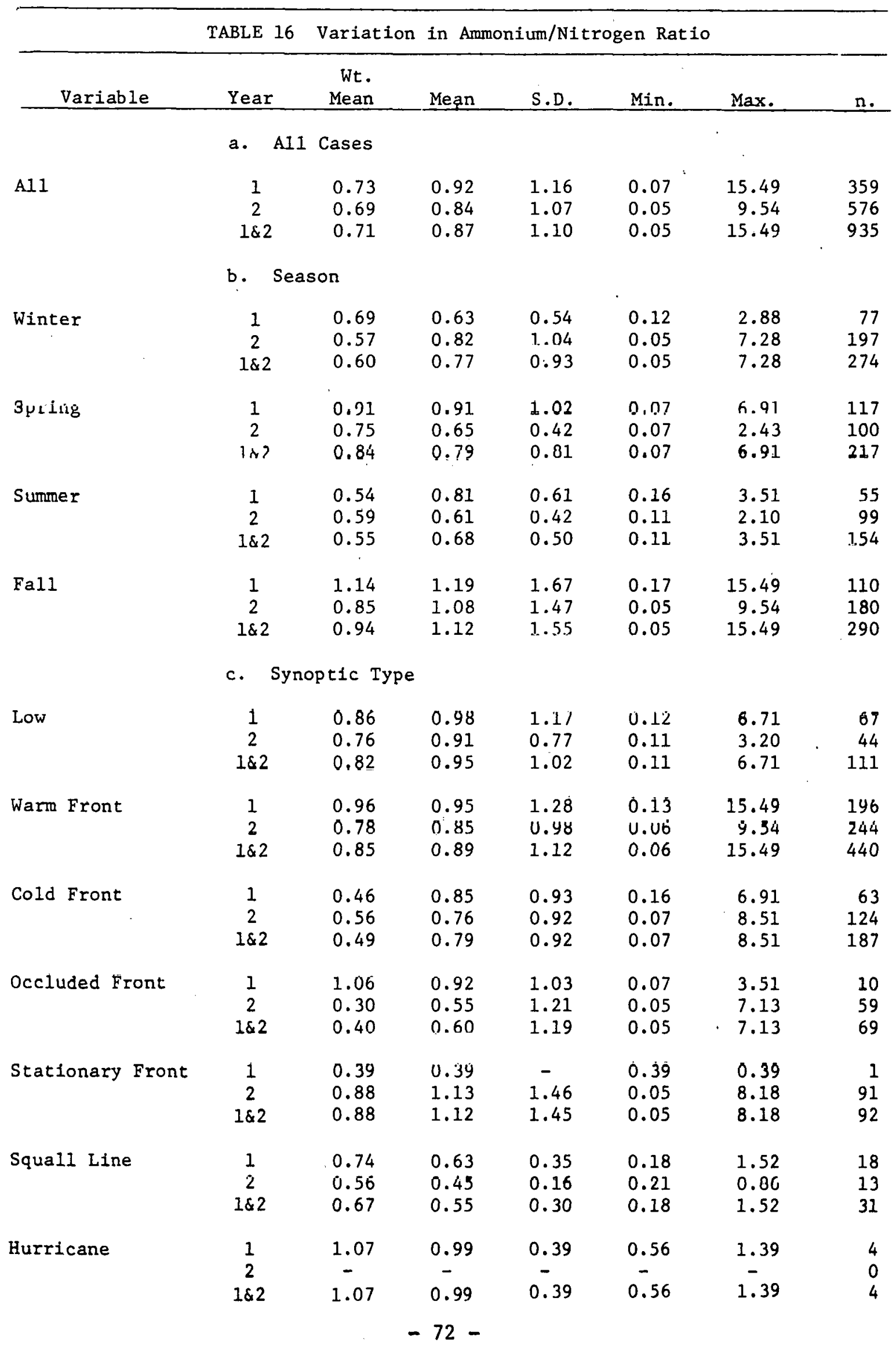


TABLE 16 (continued) Ammonium/Nitrogen

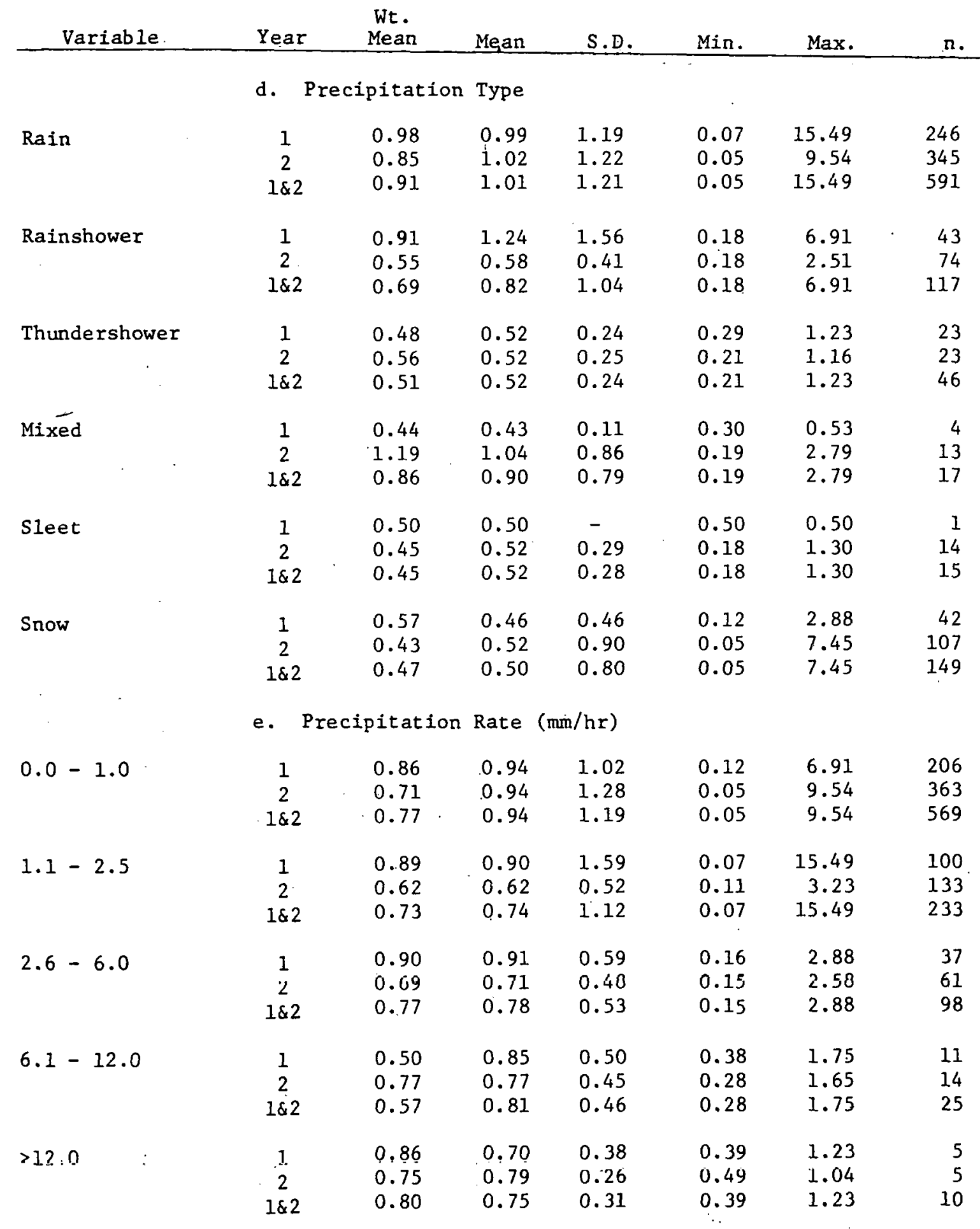


TABLE 16 (continued) Ammonium/Nitrogen

Wt.

Variable Year Mean

f. Wind Speed $\left(\mathrm{msec}^{-1}\right)$ at $108 \mathrm{~m}$

$\begin{array}{rccccccc}0-.2 & 1 & 0.48 & 0.49 & 0.38 & 0.13 & 1.48 & 17 \\ & 2 & 0.57 & 1.11 & 1.63 & 0.13 & 7.45 & 28 \\ 3-5 & 1 \& 2 & 0.52 & 0.87 & 1.33 & 0.13 & 7.45 & 45 \\ & 1 & 0.61 & 0.91 & 1.07 & 0.17 & 6.71 & 135 \\ & 2 & 0.79 & 0.90 & 1.26 & 0.05 & 9.54 & 190 \\ 6-9 & 1 \& 2 & 0.66 & 0.90 & 1.1 .8 & 0.05 & 9.54 & 325 \\ & 1 & 0.83 & 0.86 & 0.74 & 0.16 & 6.91 & 151 \\ & 2 & 0.69 & 0.82 & 0.89 & 0.05 & 8.51 & 250 \\ \geq 10 & 1 \& 2 & 0.74 & 0.83 & 0.83 & 0.05 & 8.51 & 401 \\ & & & & & & & \\ & 1 & 1.12 & 1.24 & 2.06 & 0.01 & 15.49 & 38 \\ & 2 & 0.63 & 0.74 & 0.92 & 0.06 & 7.28 & 96 \\ & 1 \& 2 & 0.01 & 0.22 & 1.46 & 0.06 & 15.19 & 151\end{array}$

g. Wind Direction at $108 \mathrm{~m}$

N

$\begin{array}{lll}1 & 0.77 & 0.70\end{array}$

20.39

0.70
0.46

0.38

0.17

1.55

33

$1 \& 2$

0.59

0.57

0.34

0.06

1.51

42

$\mathrm{NE}$

1

0.83

$0.93 \quad 1.18$

0.06

1.55

75

182

$1 \& 2$

0.73

1.01

0.17

6.71

49

0.75

0.99

1.42

0.05

9.54

210

E

1
2
$1 \& 2$

0.99

0.98

1.38

0.05

9.54

259

0.88

0.90

0.83

0.18

4.30

58

0.92

0.93

0.85

0.06

5.38

$\cdot 114$

SE

1
2
$1 \& 2$

0.53

0.90

0.64

0.06

5. 38

1.72

0.64

0.62

0.53

0.16

2.27

55

0.55

0.76

0.60

0.05

2.72

56

.1 .1 .1 .

S

$1-1.32$

1.55

2.23

0.61

0.17

2.72

53

0.67

0.71

1.64

0.17

15.49

59

SW

$1 \& 2$

0.94

1.11

0.17

15.49

112

SW 1

1
2
$1 \& 2$

0.56

0.62

0.41

0.18

2.47

0.12

8.51

46

U. 32

0.72

0.90

0.12

8.51

56

W

1

0.49

0.82

1.45

0.18

6.91

102

2
$1 \& 2$

0.64

0.81

0.77

0.07

2.98

20

0.56

0.81

1.10

0.07

6.91

26

NW

$\begin{array}{cc}1 & 0.82 \\ 2 & 0.59 \\ 1 \& 2 & 0.78\end{array}$

0.64
0.56
0.62

0.47

0.47

0.07

2.88

1.96

45

0.47

0.07

2.88 
TABLE 16 (continued) Ammonium/Nitrogen

Wt.

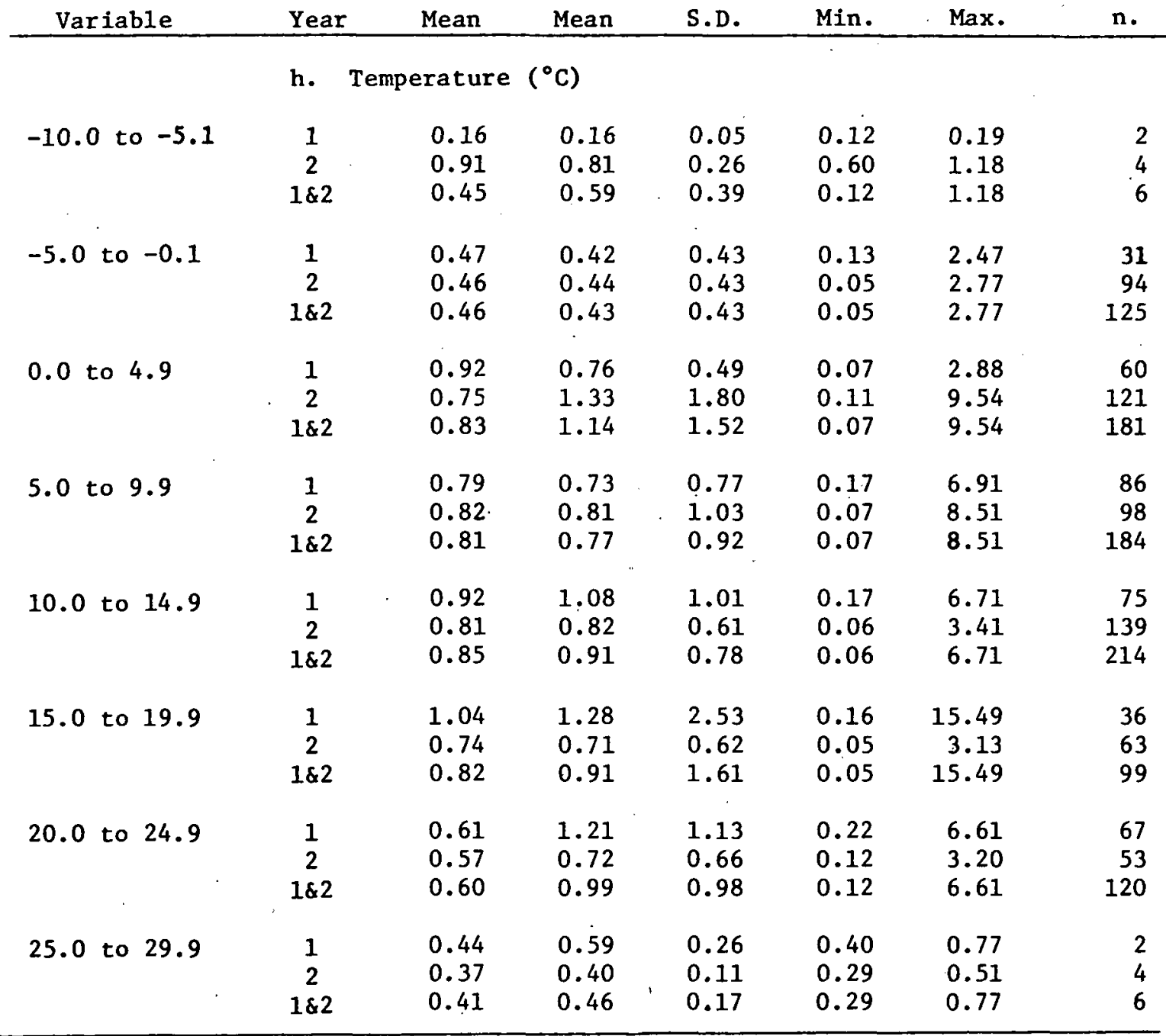




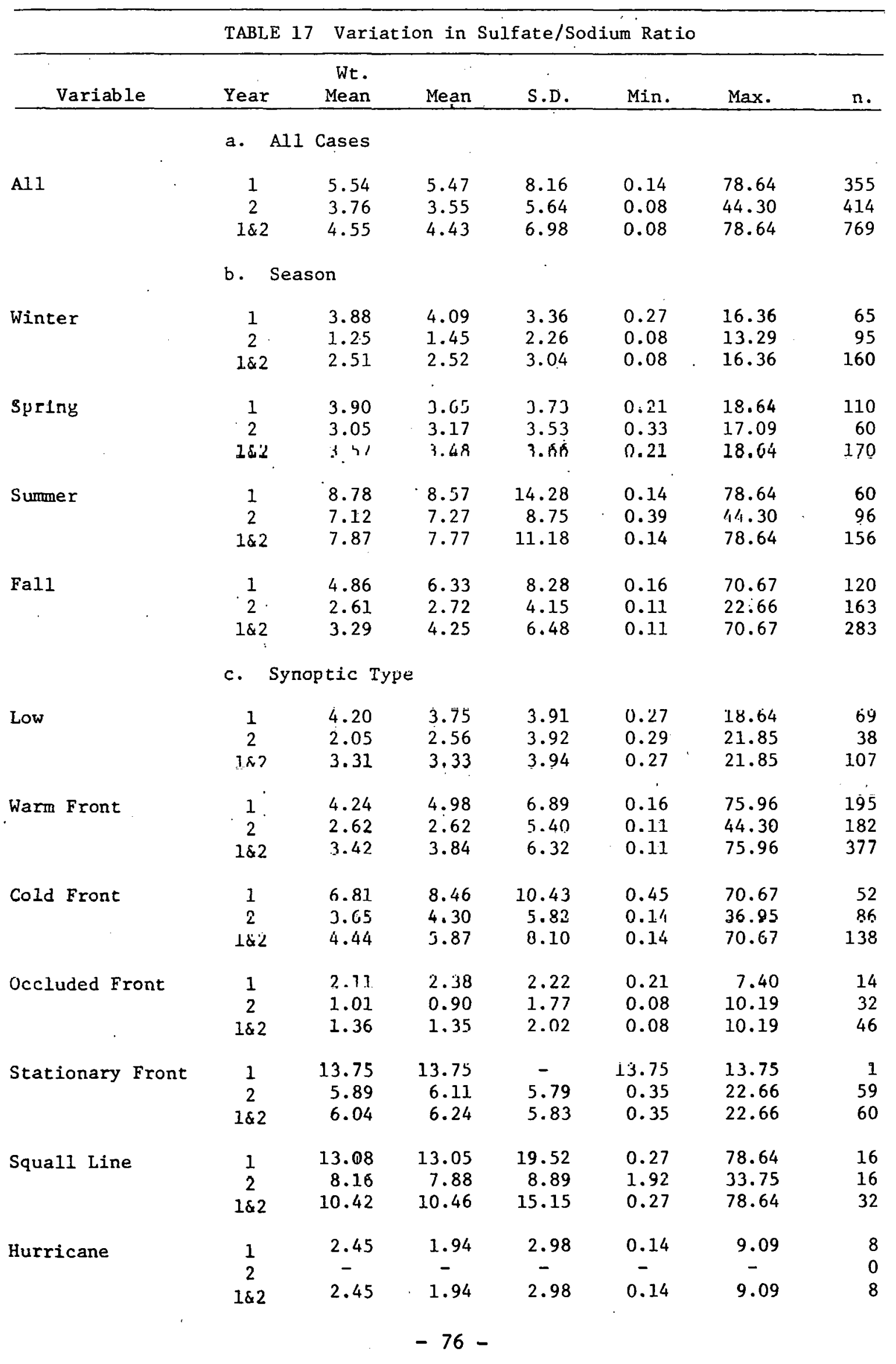


TABLE 17 (continued) Sulfate/Sodium

\begin{tabular}{lllll} 
Variable & Year & Mean & Mean $\quad$ S.D. Min. Max. M. M. \\
\hline
\end{tabular}

d. Precipitation Type

Rain

$\begin{array}{lll}1 & 4.18 & 4.57 \\ 2 & 2.64 & 2.88\end{array}$

6.39

$0.15 \quad 75.96$

263

$1 \& 2$

3.33

3.68

4.94

0.11

44.30

294

1
2
$1 \& 2$

5.73

$10.18 \quad 16.33$

0.11

75.96

557

Rainshower

6.71

7.52

7.67

0.27

78.64

$0.36 \quad 36.95$

38

6.25

$8.71 \quad 12.30$

$0.27 \quad 78.64$

47

85

Thundershower

$\begin{array}{cr}1 & 10.06 \\ 2 & 6.82 \\ 1 \& 2 & 8.22\end{array}$

$8.94 \quad 7.74$

0.14

30.58

25

$.6 .34 \quad 8.12$

$0.89 \quad 33.75$

24

$1 \notin 2$

Mixed

$\begin{array}{cc}1 & 7.74 \\ 2 & 3.23 \\ 1 \& 2 & .6 .32\end{array}$

6.77
3.88

7.9 .5

$0.14 \quad 33.75$

49

sleet

$\begin{array}{ccccccc}1 & - & - & - & - & - & 0 \\ 2 & 1.48 & 2.67 & 4.52 & 0.29 & 11.87 & 6 \\ 1 \& 2 & 1.48 & 2.67 & 4.52 & 0.29 & 11.87 & 6\end{array}$

Snow

$\begin{array}{ccccccc}1 & 4.30 & 4.19 & 2.50 & 0.69 & 10.91 & 26 \\ 2 & 1.82 & 2.21 & 3.58 & 0.08 & 17.09 & 40 \\ 1 \& 2 & 2.82 & 2.99 & 3.32 & 0.08 & 17.09 & 66\end{array}$

e. Precipitation Rate (mm/hr)

\begin{tabular}{|c|c|c|c|c|c|c|c|c|}
\hline $0.0-1.0$ & . & $\begin{array}{c}1 \\
2 \\
1 \& 2\end{array}$ & $\begin{array}{l}6.53 \\
3.14 \\
4.62\end{array}$ & $\begin{array}{l}6.63 \\
3.94 \\
5.22\end{array}$ & $\begin{array}{r}10.17 \\
5.83 \\
8.29\end{array}$ & $\begin{array}{l}0.15 \\
0.08 \\
0.08\end{array}$ & $\begin{array}{l}78.64 \\
44.30 \\
78.64\end{array}$ & $\begin{array}{l}186 \\
204 \\
390\end{array}$ \\
\hline $\begin{array}{c}1.1-2.5 \\
.\end{array}$ & . & $\begin{array}{c}1 \\
2 \\
1 \& 2\end{array}$ & $\begin{array}{l}3.87 \\
2.86 \\
3.31\end{array}$ & $\begin{array}{r}3.97 \\
3.03 \\
.3 .46\end{array}$ & $\begin{array}{l}5.14 \\
5.92 \\
5.59\end{array}$ & $\begin{array}{l}0.18 \\
0.14 \\
0.14\end{array}$ & $\begin{array}{l}30.58 \\
43.81 \\
43.81\end{array}$ & $\begin{array}{r}98 \\
119 \\
217\end{array}$ \\
\hline $2.6-6.0$ & . & $\begin{array}{c}1 \\
2 \\
1 \& 2\end{array}$ & $\begin{array}{l}3.49 \\
3.13 \\
3.28\end{array}$ & $\begin{array}{r}3.41 \\
.3 .92 \\
3.13\end{array}$ & $\begin{array}{r}2.93 \\
3.59 \\
.3 .32\end{array}$ & $\begin{array}{l}0.34 \\
0.11 \\
0.11\end{array}$ & $\begin{array}{l}14.15 \\
19.54 \\
19.54\end{array}$ & $\begin{array}{r}47 \\
63 \\
110\end{array}$ \\
\hline $6.1-12.0$ & , & $\begin{array}{c}1 \\
2 \\
1 \& 2\end{array}$ & $\begin{array}{l}4.85 \\
3.38 \\
4 . .01\end{array}$ & $\begin{array}{l}4.80 \\
3.08 \\
3.81\end{array}$ & $\begin{array}{l}3.65 \\
3.50 \\
3.62\end{array}$ & $\begin{array}{l}0.14 \\
0.18 \\
0.14\end{array}$ & $\begin{array}{l}11.48 \\
15.08 \\
15.08\end{array}$ & $\begin{array}{l}16 \\
22 \\
38\end{array}$ \\
\hline$>12.0$ & . & $\begin{array}{c}1 \\
2 \\
1 \& 2\end{array}$ & $\begin{array}{r}11.27 \\
/ .9 / \\
9.57\end{array}$ & $\begin{array}{r}10.21 \\
8.82 \\
: 9.62\end{array}$ & $\begin{array}{r}7.88 \\
12.61 \\
9.75\end{array}$ & $\begin{array}{l}1.46 \\
1.26 \\
1.26\end{array}$ & $\begin{array}{l}22.65 \\
33.75 \\
33.75\end{array}$ & $\begin{array}{r}8 \\
6 \\
14\end{array}$ \\
\hline
\end{tabular}


TABLE 17 (continued) Sulfate/Sodium

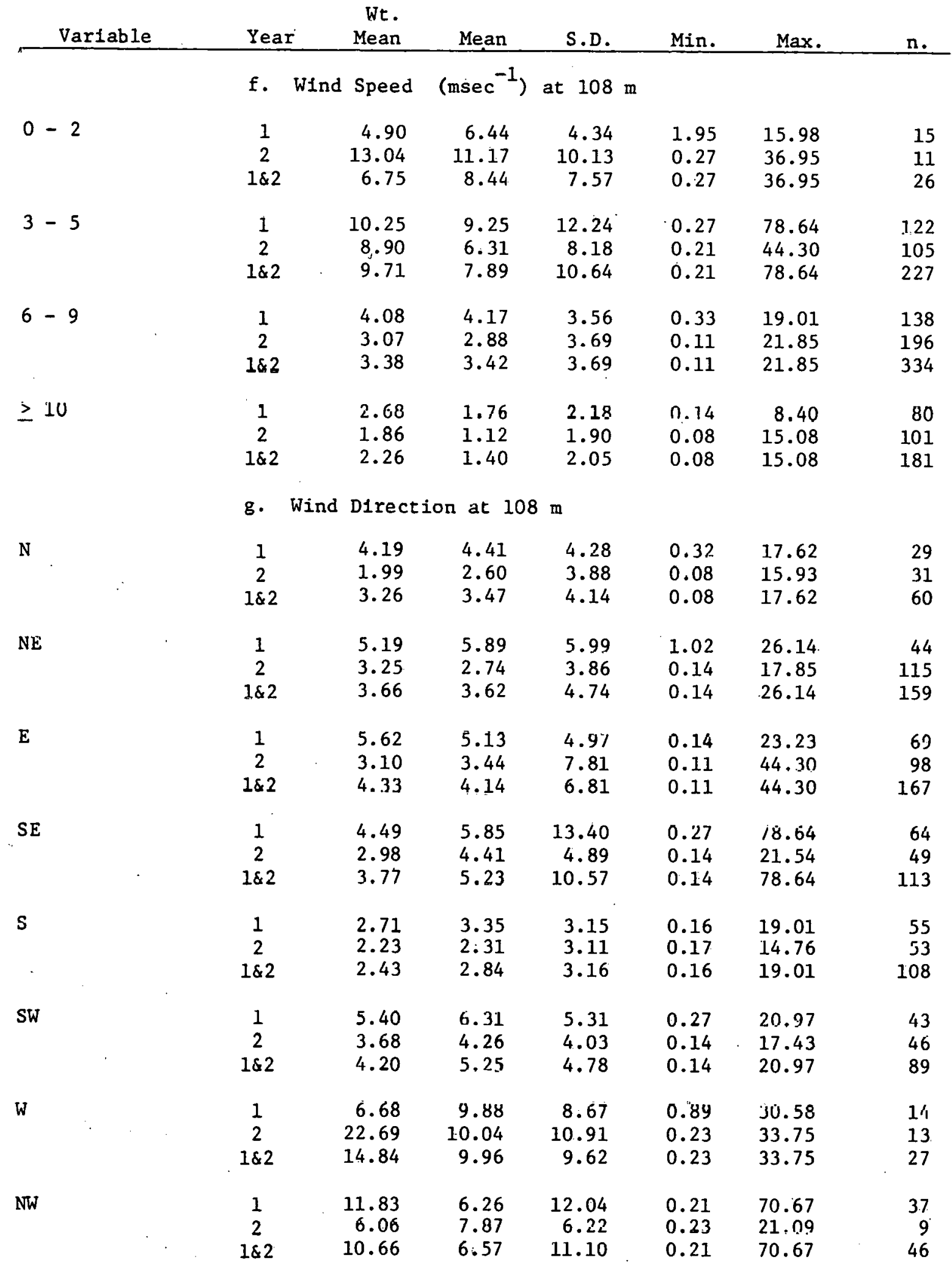


TABLE 17 (continued) Sulfate/Sodium

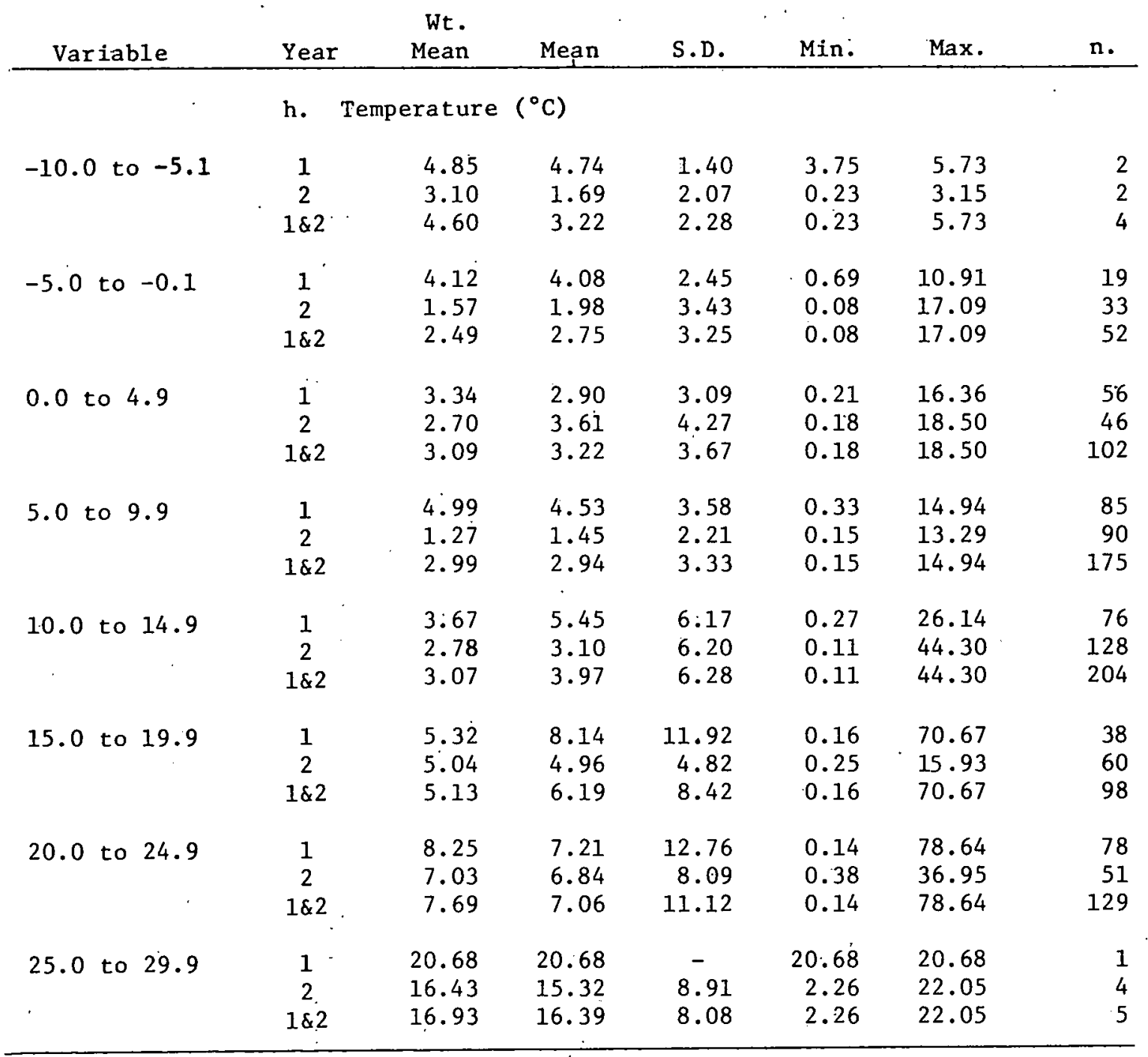




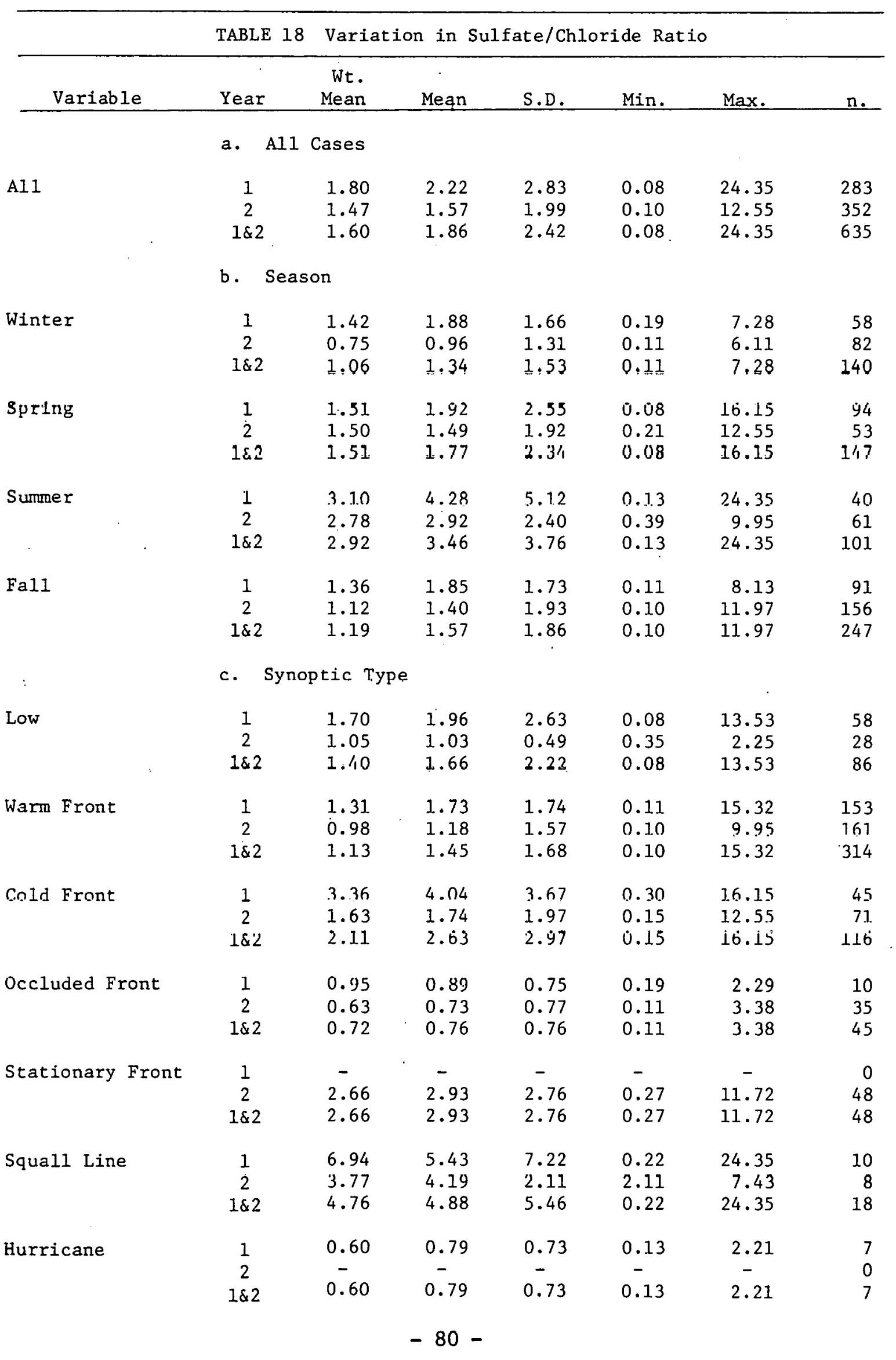


TABLE 18 (continued) Sulfate/Chloride

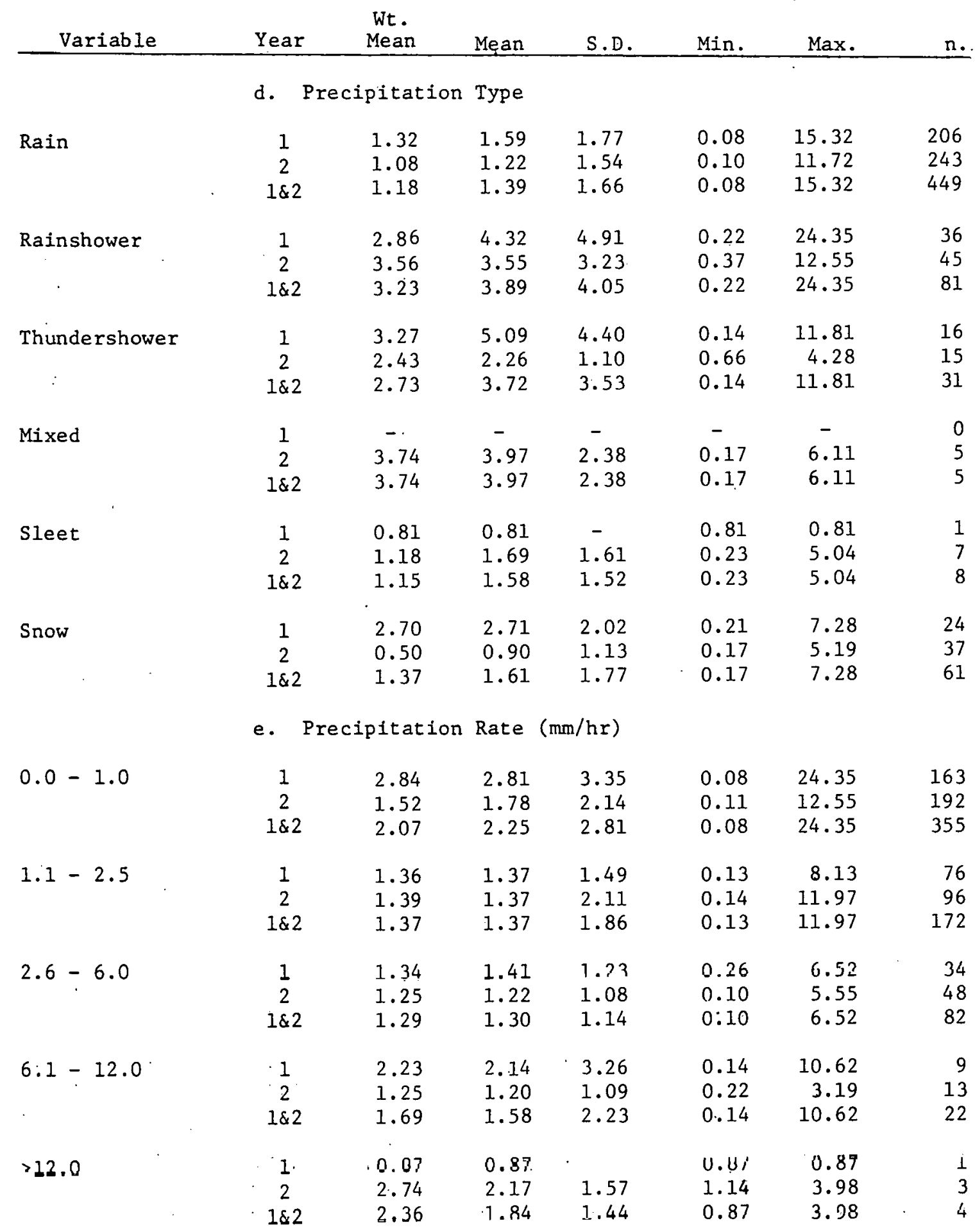


TABLE 18 (continued) Sulfate/Chloride

Wt.

\begin{tabular}{|c|c|c|c|c|c|c|c|}
\hline Variable & Year & Mean & Mean & S.D. & Min. & Max. & $\mathrm{n}$. \\
\hline & f. & Speed & (msec $^{-}$ & 108 & & & \\
\hline $0-2$ & $\begin{array}{c}1 \\
2 \\
1 \& 2\end{array}$ & $\begin{array}{l}2.40 \\
2.86 \\
2.59\end{array}$ & $\begin{array}{l}2.72 \\
3.13 \\
: 2.94\end{array}$ & $\begin{array}{l}1.02 \\
2.55 \\
1.97\end{array}$ & $\begin{array}{l}1.65 \\
0.23 \\
0.23\end{array}$ & $\begin{array}{l}5.21 \\
8.36 \\
8.36\end{array}$ & $\begin{array}{l}10 \\
12 \\
22\end{array}$ \\
\hline $3-5$ & $\begin{array}{c}1 \\
2 \\
1 \& 2\end{array}$ & $\begin{array}{l}3.75 \\
2.67 \\
3.30\end{array}$ & $\begin{array}{l}3.65 \\
2.75 \\
3.20\end{array}$ & $\begin{array}{l}3.90 \\
2.93 \\
3.47\end{array}$ & $\begin{array}{l}0.22 \\
0.11 \\
0.11\end{array}$ & $\begin{array}{l}24.35 \\
12.55 \\
24.35\end{array}$ & $\begin{array}{r}80 \\
79 \\
159\end{array}$ \\
\hline $6-9$ & $\begin{array}{c}1 \\
2 \\
1 \& 2\end{array}$ & $\begin{array}{l}1.88 \\
1.60 \\
1.69\end{array}$ & $\begin{array}{l}2.15 \\
1.37 \\
1.70\end{array}$ & $\begin{array}{l}2.33 \\
1.54 \\
1.95\end{array}$ & $\begin{array}{l}0.16 \\
0.10 \\
0.10\end{array}$ & $\begin{array}{r}16.15 \\
9.95 \\
16.15\end{array}$ & $\begin{array}{l}119 \\
165 \\
284\end{array}$ \\
\hline$\geq 10$ & $\begin{array}{c}1 \\
2 \\
1 \& 2\end{array}$ & $\begin{array}{l}0.77 \\
0.82 \\
0.80\end{array}$ & $\begin{array}{l}0.13 \\
0.68 \\
0.70\end{array}$ & $\begin{array}{l}0.98 \\
0.56 \\
0.77\end{array}$ & $\begin{array}{l}0.08 \\
0.13 \\
0.18\end{array}$ & $\begin{array}{l}7.28 \\
2.46 \\
7.28\end{array}$ & $\begin{array}{r}74 \\
94 \\
168\end{array}$ \\
\hline
\end{tabular}

g. Wind Direction at $108 \mathrm{~m}$

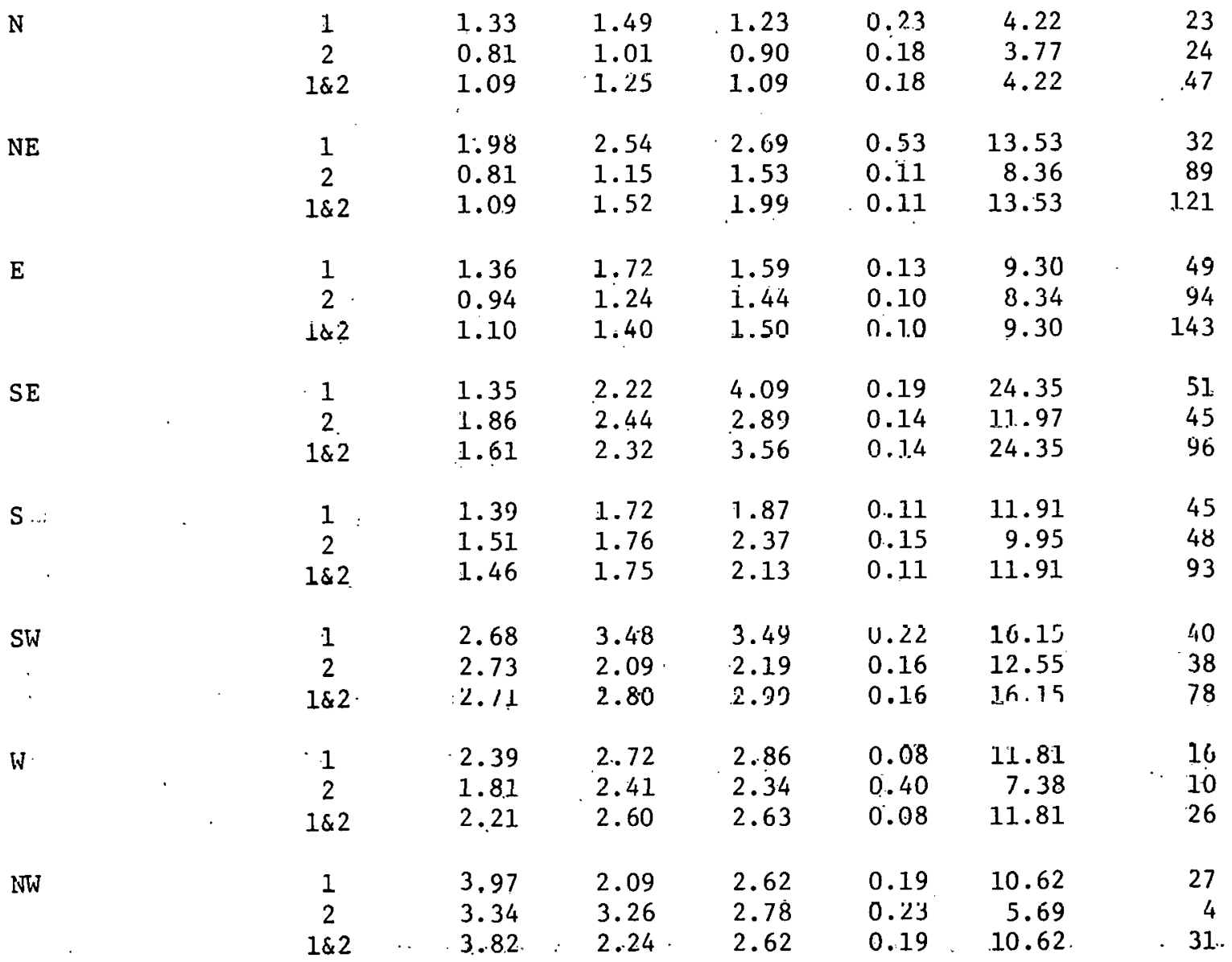


TABLE 18 (continued) Sulfate/Chloride

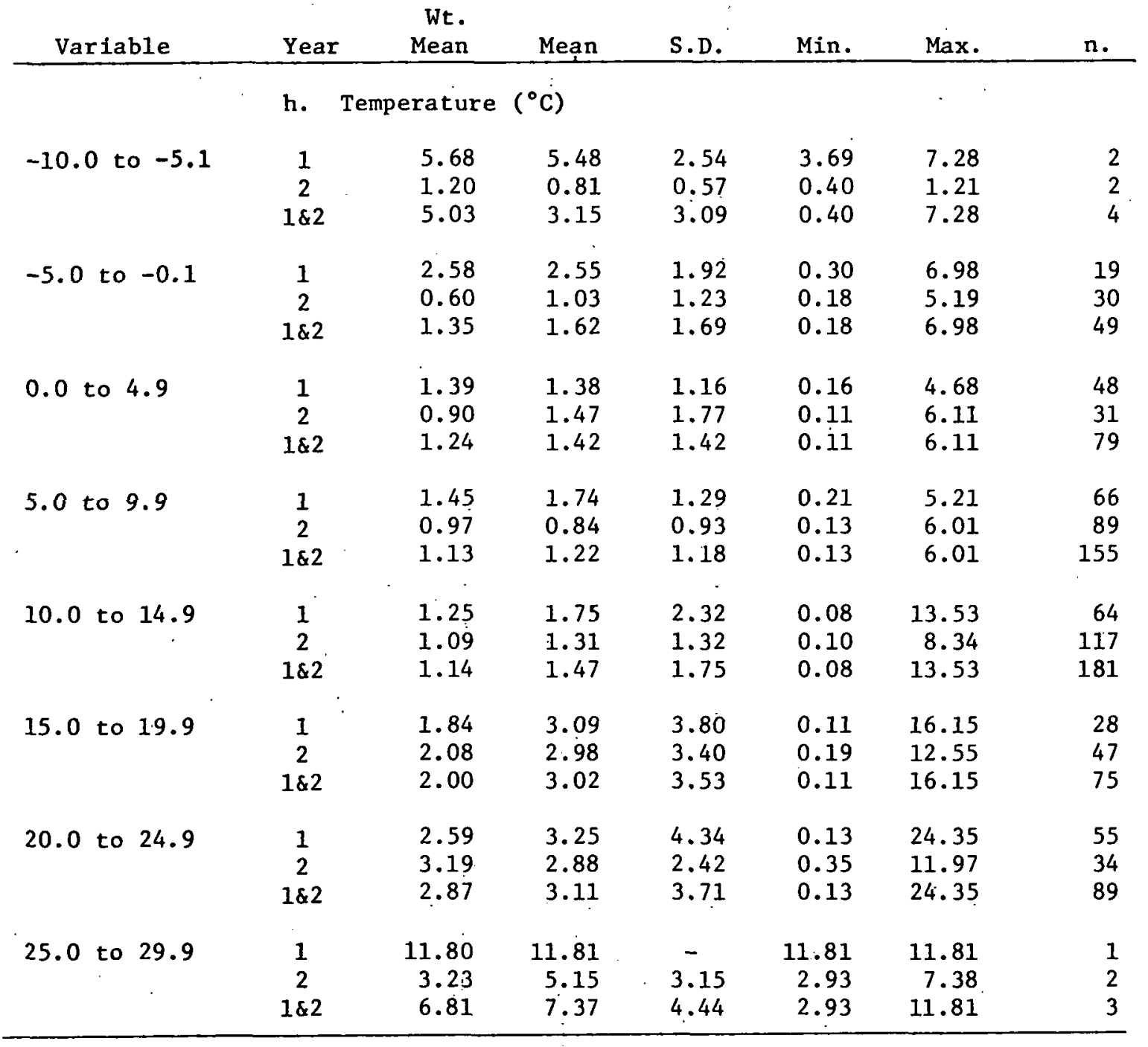




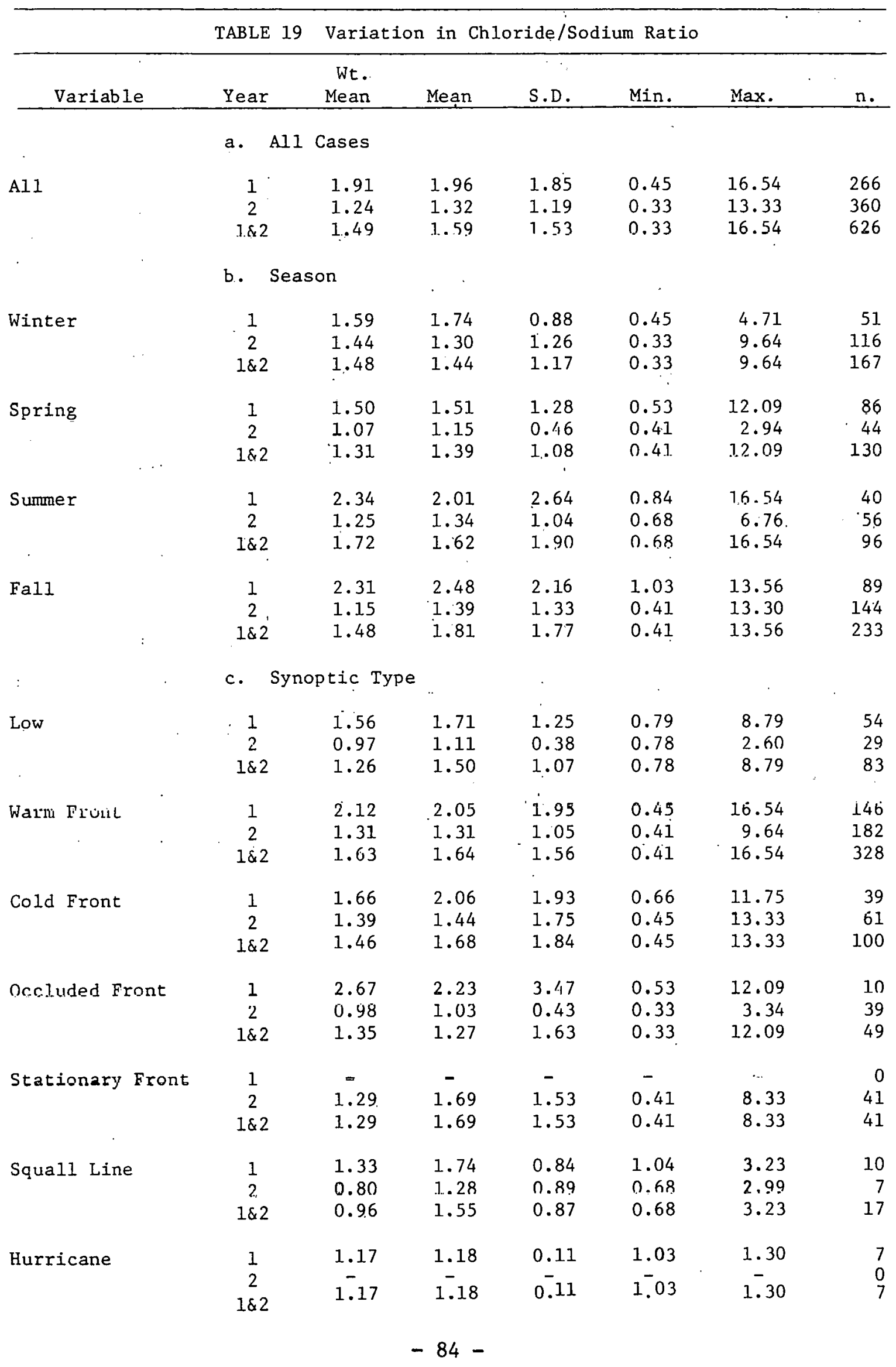


TABLE 19 (continued) Chloride/Sodium

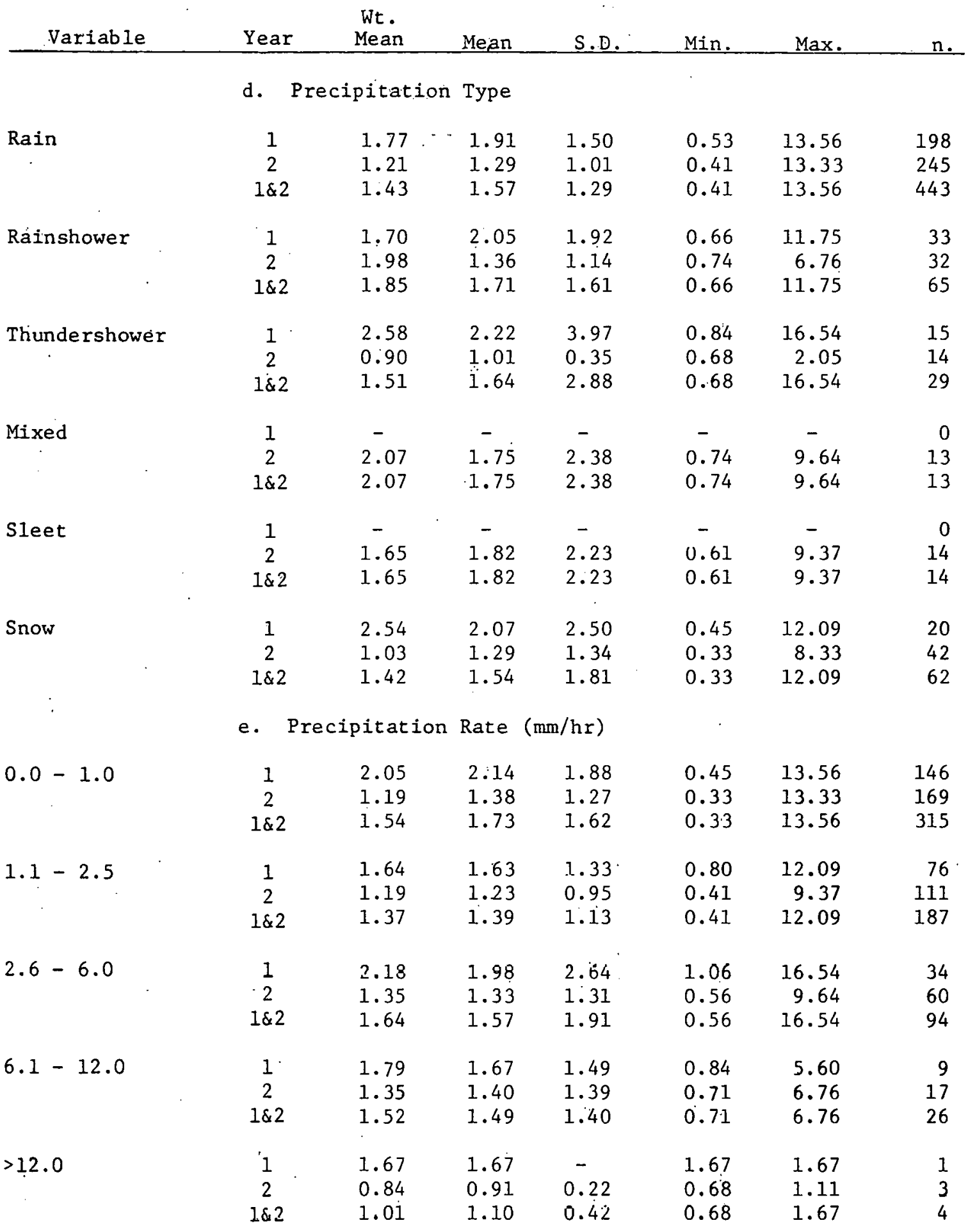


TABLE 19 (continued) Chloride/Sodium

Wt.

\begin{tabular}{|c|c|c|c|c|c|c|c|}
\hline Variable & Year & Mean & Mean & S.D. & Min. & Max. & n. \\
\hline & f. $\quad$ & Speed & (m sec $^{-1}$ & at $108 \mathrm{~m}$ & & . & \\
\hline $0-2$ & $\begin{array}{c}1 \\
2 \\
1 \& 2\end{array}$ & $\begin{array}{l}2.66 \\
2.25 \\
2.54\end{array}$ & $\begin{array}{l}2.61 \\
3.41 \\
2.94\end{array}$ & $\begin{array}{l}2.02 \\
2.71 \\
2.29\end{array}$ & $\begin{array}{l}0.91 \\
0.74 \\
0.74\end{array}$ & $\begin{array}{l}5.91 \\
8.33 \\
8.33\end{array}$ & $\begin{array}{r}10 \\
7 \\
17\end{array}$ \\
\hline $3-5$ & $\begin{array}{c}1 \\
2 \\
1 \& 2\end{array}$ & $\begin{array}{l}2.80 \\
1.37 \\
2.21\end{array}$ & $\begin{array}{l}2.50 \\
1.61 \\
2.08\end{array}$ & $\begin{array}{l}2.73 \\
1.69 \\
2.34\end{array}$ & $\begin{array}{l}0.45 \\
0.45 \\
0.45\end{array}$ & $\begin{array}{l}16.54 \\
13.33 \\
16.54\end{array}$ & $\begin{array}{r}78 \\
68 \\
146\end{array}$ \\
\hline $6-9$ & $\begin{array}{c}1 \\
2 \\
1 \& 2\end{array}$ & $\begin{array}{l}1.85 \\
1.29 \\
1.45\end{array}$ & $\begin{array}{l}1.80 \\
1.29 \\
1.49\end{array}$ & $\begin{array}{l}1.09 \\
0.94 \\
1.03\end{array}$ & $\begin{array}{l}0.70 \\
0.41 \\
0.41\end{array}$ & $\begin{array}{l}7.21 \\
9.37 \\
9.37\end{array}$ & $\begin{array}{l}109 \\
164 \\
273\end{array}$ \\
\hline$\geq 10$ & $\begin{array}{c}1 \\
2 \\
1 \& 2\end{array}$ & $\begin{array}{l}1.50 \\
1.04 \\
1.23\end{array}$ & $\begin{array}{l}1.30 \\
1.03 \\
1.21\end{array}$ & $\begin{array}{l}1.33 \\
0.27 \\
0.87\end{array}$ & $\begin{array}{l}0.53 \\
0.33 \\
0.33\end{array}$ & $\begin{array}{r}12.09 \\
2.30 \\
12.09\end{array}$ & $\begin{array}{r}69 \\
112 \\
181\end{array}$ \\
\hline
\end{tabular}

g. Wind Direction at $108 \mathrm{~m}$

$\mathbf{N}$

$\begin{array}{ccccccc}1 & 1.69 & 1.77 & 0.67 & 1.11 & 3.71 & 22 \\ 2 & 1.00 & 1.18 & 0.75 & 0.33 & 4.2 .3 & 24 \\ 1 \& 2 & 1.37 & 1.46 & 0.77 & 0.33 & 4.23 & 46\end{array}$

NE

$\begin{array}{rrrrrrr}1 & 1.81 & 1.73 & 0.96 & 0.84 & 5.26 & 30 \\ 2 & 1.33 & 1.33 & 1.26 & 0.53 & 9.64 & 113\end{array}$

E

$1 \& 2$

1.41

1.41

1.21

0.53

9. 64

143

$\begin{array}{crrrrrr}1 & 2.29 & 2.23 & 2.59 & 0.45 & 16.54 & 46 \\ 2 & 1.33 & 1.41 & 1.12 & 0.41 & 8.33 & 93 \\ 1 . \$ 2 & 1.67 & 1.68 & 1.78 & 0.41 & 16.54 & 139\end{array}$

SE

$\begin{array}{ccccccc}1 & 1.99 & 1.99 & 1.70 & 0.66 & 8.79 & 51 \\ 2 & 1.03 & 1.10 & 0.30 & 0.41 & 1.81 & 41 \\ 1 \& 2 & 1.49 & 1.60 & 1.35 & 0.41 & 8.79 & 92\end{array}$

S

$\begin{array}{crrrrrr}1 & 1.59 & 1.75 & 1.89 & 0.80 & 13.56 & 44 \\ 2 & 1.44 & 1.31 & 0.88 & 0.56 & 6.76 & 47 \\ 1 \& 2 & 1.50 & 1.52 & 1.47 & 0.56 & 13.56 & 91\end{array}$

\begin{tabular}{|c|c|c|c|c|c|c|c|}
\hline SW & $\begin{array}{c}1 \\
\dot{2} \\
1 \dot{\&} 2\end{array}$ & $\begin{array}{l}1.67 \\
0.99 \\
1.18\end{array}$ & $\begin{array}{l}1.77 \\
1.53 \\
1.66\end{array}$ & $\begin{array}{l}0.82 \\
2.20 \\
1.62\end{array}$ & $\begin{array}{l}0.84 \\
0.68 \\
0.68\end{array}$ & $\begin{array}{r}4.09 \\
13.93 \\
13.33\end{array}$ & $\begin{array}{l}35 \\
32 \\
67\end{array}$ \\
\hline $\mathrm{W}$ & $\begin{array}{c}1 \\
2 \\
1 \& 2\end{array}$ & $\begin{array}{l}3.14 \\
0.95 \\
2.49\end{array}$ & $\begin{array}{l}3.62 \\
1.11 \\
2.58\end{array}$ & $\begin{array}{l}3.19 \\
0.88 \\
2.77\end{array}$ & $\begin{array}{l}1.29 \\
0.45 \\
0.45\end{array}$ & $\begin{array}{r}12.09 \\
2.99 \\
12.09\end{array}$ & $\begin{array}{r}10 \\
7 \\
17\end{array}$ \\
\hline NW & $\begin{array}{c}1 \\
2 \\
1 \& 2\end{array}$ & $\begin{array}{l}1.38 \\
1.01 \\
1.30\end{array}$ & $\begin{array}{l}1.80 \\
1.01 \\
1.72\end{array}$ & $\begin{array}{l}2.09 \\
0.18 \\
2.00\end{array}$ & $\begin{array}{l}0.53 \\
0.82 \\
0.53\end{array}$ & $\begin{array}{r}11.75 \\
1.19 \\
11.75\end{array}$ & $\begin{array}{r}28 \\
3 \\
31\end{array}$ \\
\hline
\end{tabular}


TABLE 19 (continued) Chloride/Sodium

\begin{tabular}{|c|c|c|c|c|c|c|c|}
\hline Variable & Year & $\begin{array}{l}\text { Wt. } \\
\text { Mean }\end{array}$ & Mean & S.D. & Min. & Max. & n. \\
\hline & h. $T$ & rature & $\left.{ }^{\circ} \mathrm{C}\right)$ & & & & \\
\hline \multirow[t]{3}{*}{-10.0 to -5.1} & 1 & 0.89 & 0.90 & 0.16 & 0.79 & 1.02 & \\
\hline & 2 & 2.57 & 1.58 & 1.44 & 0.57 & 2.60 & \\
\hline & $1 \& 2$ & $1: 13$ & 1.24 & 0.92 & 0.57 & 2.60 & 4 \\
\hline \multirow[t]{3}{*}{-5.0 to -0.1} & 1 & 1.55 & 1.60 & 0.94 & 0.45 & 3.69 & 14 \\
\hline & 2 & 1.15 & 1.18 & 1.25 & 0.33 & 9.37 & 48 \\
\hline & $1 \& 2$ & 1.21 & 1.28 & 1.19 & 0.33 & 9.37 & 62 \\
\hline \multirow[t]{3}{*}{0.0 to 4.9} & 1 & 1.63 & 1.61 & 1.61 & 0.53 & 12.09 & 49 \\
\hline & 2 & 1.82 & 1.89 & 1.95 & 0.45 & 9.64 & 39 \\
\hline & $1 \& 2$ & 1.72 & 1.74 & 1.76 & 0.45 & 12.08 & 88 \\
\hline \multirow[t]{3}{*}{5.0 to 9.9} & 1 & 1.61 & 1.70 & 0.77 & 0.66 & 4.71 & 58 \\
\hline & 2 & 1.22 & 1.18 & 0.64 & 0.53 & 6.38 & 83 \\
\hline & $1 \& 2$ & $1: 33$ & 1.39 & 0.74 & 0.53 & 6.38 & 141 \\
\hline \multirow[t]{3}{*}{10.0 to 14.9} & 1 & 2.08 & 2.39 & 2.16 & 0.89 & 13.56 & 60 \\
\hline & 2 & 1.10 & 1.18 & 0.58 & 0.41 & 5.26 & 109 \\
\hline & $1 \& 2$ & 1.43 & 1.61 & 1.48 & 0.41 & 13.56 & 169 \\
\hline \multirow[t]{3}{*}{15.0 to 19.9} & 1 & 1.82 & 2.41 & 2.26 & 1.13 & 11.75 & 26 \\
\hline & 2 & 1.71 & 1.47 & 0.99 & 0.41 & 6.76 & 44 \\
\hline & $1 \& 2$ & 1.75 & 1.82 & 1.64 & 0.41 & 11.75 & 70 \\
\hline \multirow[t]{3}{*}{20.0 to 24.9} & 1 & 2.23 & 1.99 & 2.35 & 0.84 & 16.54 & 56 \\
\hline & 2 . & 0.95 & 1.47 & 2.17 & 0.68 & 13.33 & 33 \\
\hline & $1 \& 2$ & 1.63 & 1.80 & 2.29 & 0.68 & 16.54 & 89 \\
\hline \multirow[t]{3}{*}{25.0 to 29.9} & 1 & 1.75 & 1.75 & - & 1.75 & 1.75 & 1 \\
\hline & 2 & 0.92 & 1.88 & 1.57 & 0.77 & 2.99 & 2 \\
\hline & $1 \& 2$ & 1.27 & 1.84 & 1.11 & 0.77 & 2.99 & 3 \\
\hline
\end{tabular}




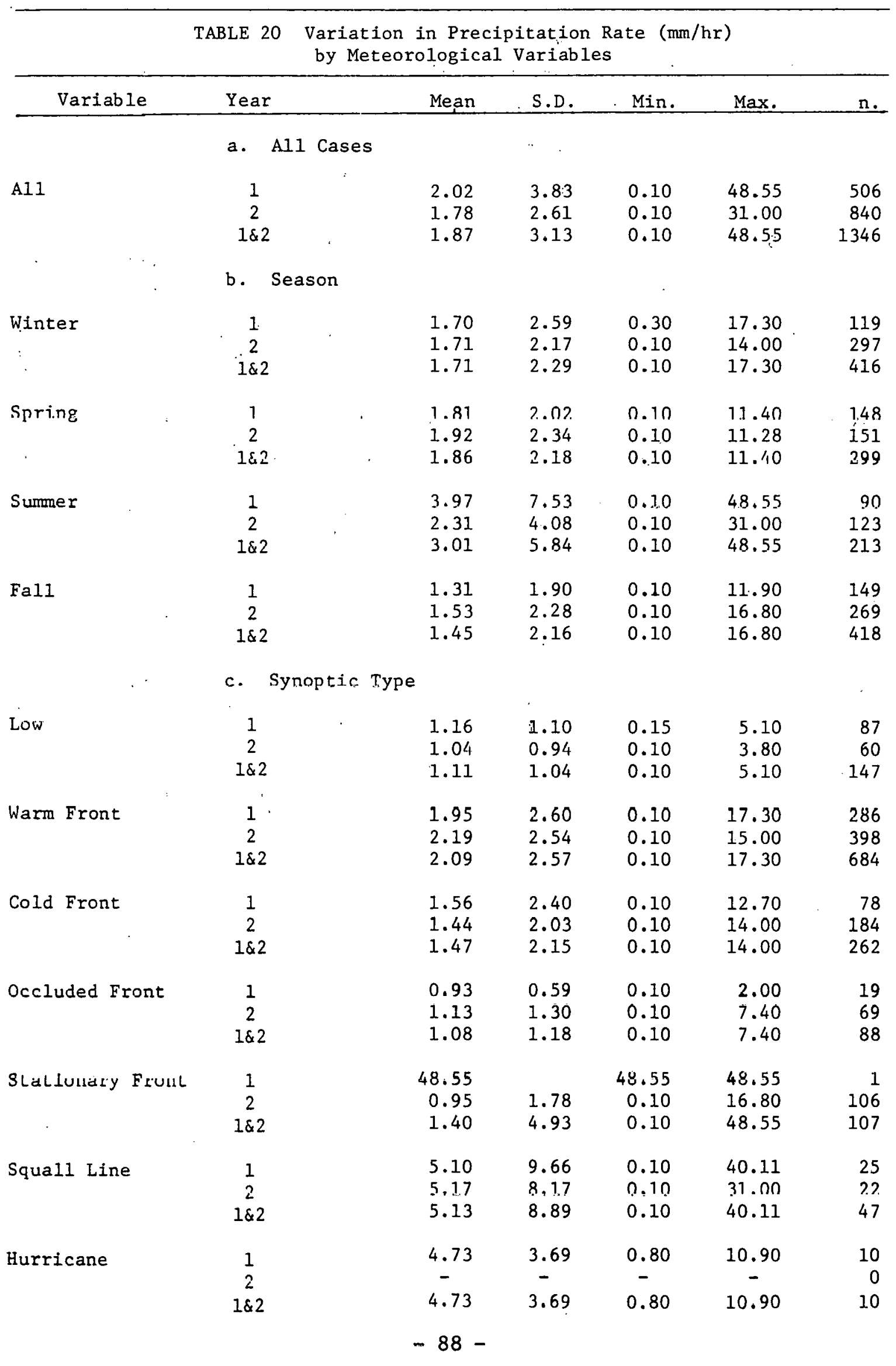


TABLE 20 (continued) Precipitation Rate

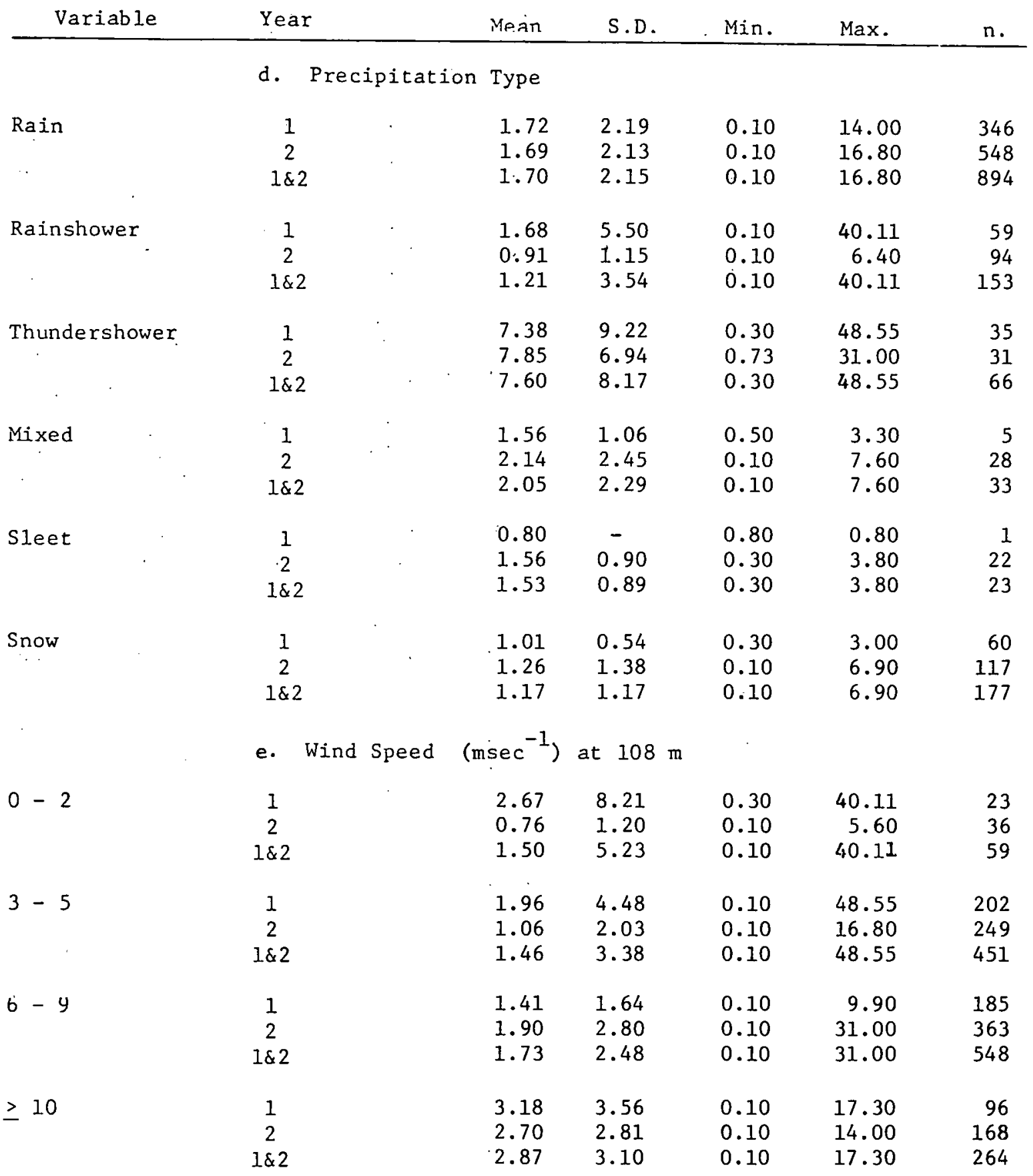


TABLE 20 (continued) Precipitation Rate

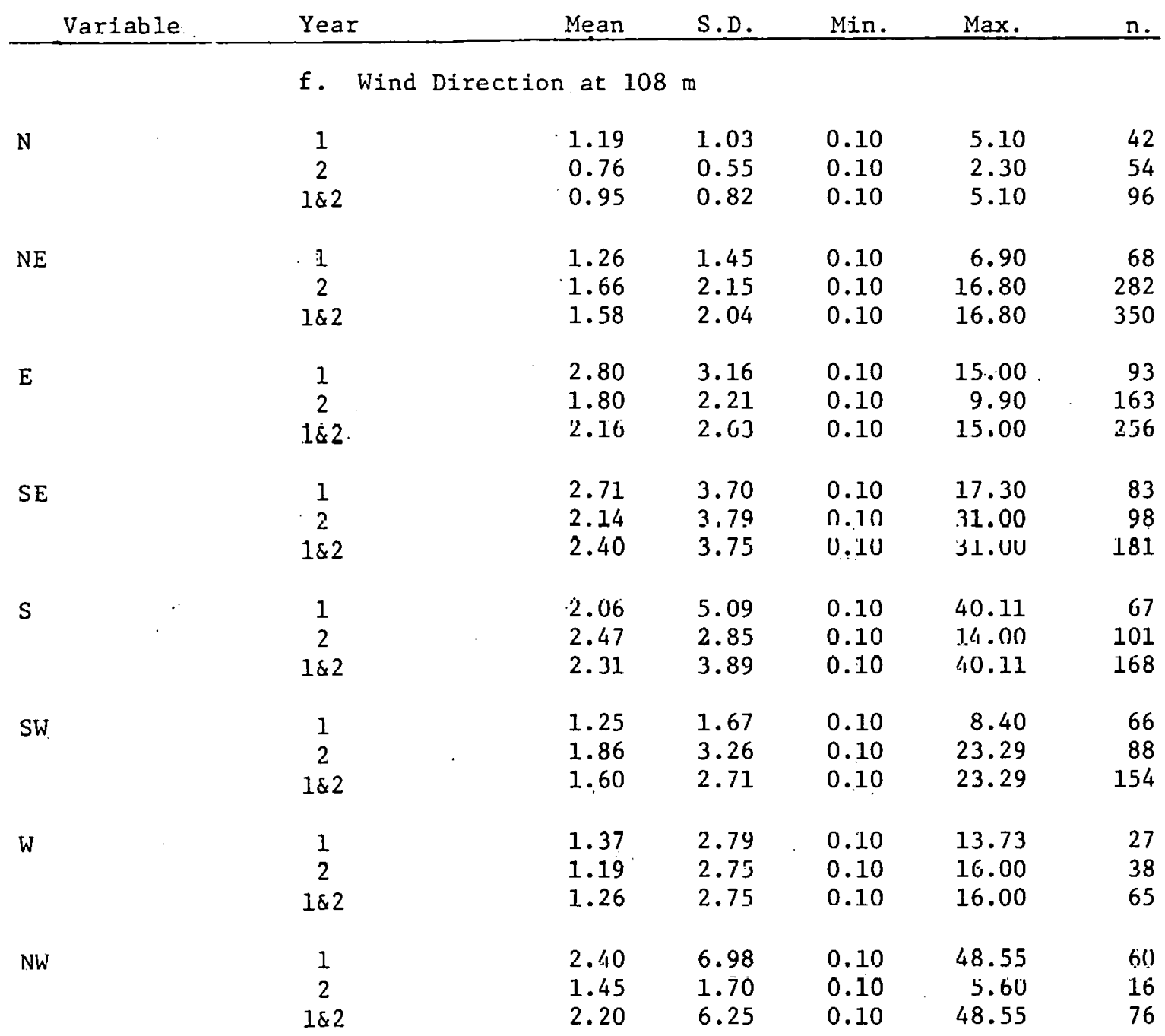


TABLE 20 (continued) Precipitation Rate

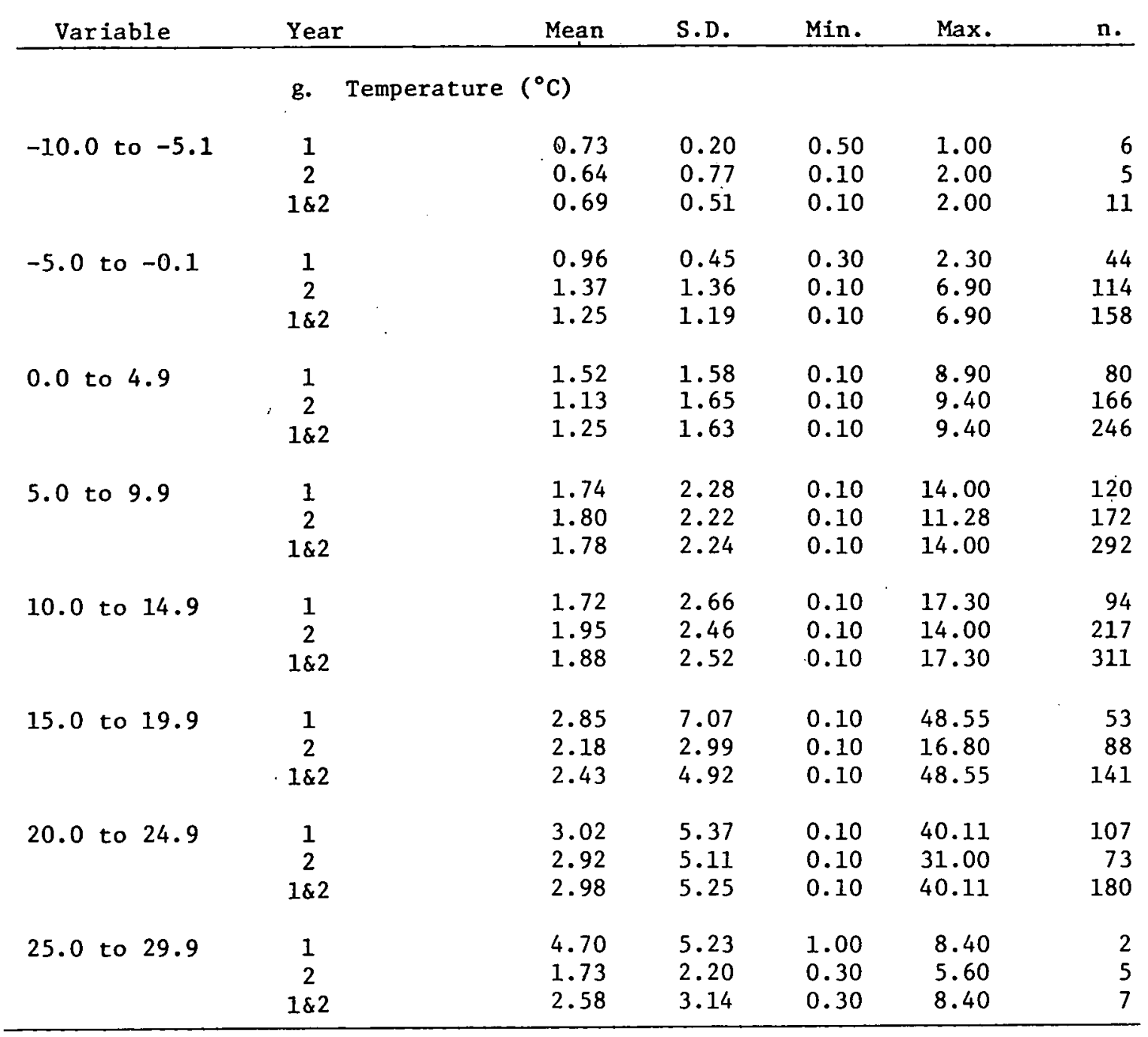

a U.S. GOVERNMENT PRINTING OFFICE: 1980-614-090/11 